\title{
Massive Star Cluster Formation and Destruction in Luminous Infrared Galaxies in GOALS
}

\author{
S. T. Linden ${ }^{1}$, A. S. Evans ${ }^{1,2}$, J. Rich ${ }^{3}$, K. L. Larson ${ }^{3}$, L. Armus ${ }^{3}$, T. Díaz-Santos ${ }^{4}$, G. C. Privon ${ }^{5}$, J. Howell ${ }^{3}$, H. Inami ${ }^{6}$, D.-C. Kim ${ }^{2}$, \\ L.-H. Chien ${ }^{7}$, T. Vavilkin ${ }^{8}$, J. M. Mazzarella ${ }^{9}$, F. Modica ${ }^{8}$, J. A. Surace ${ }^{10}$, S. Manning ${ }^{11,14}$, A. Abdullah ${ }^{12,14}$, A. Blake ${ }^{12,14}$, \\ A. Yarber ${ }^{12,14}$, and T. Lambert ${ }^{13,14}$ \\ ${ }^{1}$ Astronomy Department, University of Virginia, 530 McCormick Road, Charlottesville, VA 22904, USA; st17ey@ virginia.edu \\ ${ }^{2}$ National Radio Astronomy Observatory, 520 Edgemont Road, Charlottesville, VA 22903, USA \\ ${ }^{3}$ Infrared Processing and Analysis Center, California Institute of Technology, MS 100-22, Pasadena, CA 91125, USA \\ ${ }^{4}$ Núcleo de Astronomía de la Facultad de Ingeniería, Universidad Diego Portales, Av. Ejército Libertador 441, Santiago, Chile \\ ${ }^{5}$ Instituto de Astrofísica, Facultad de Física, Pontificia Universidad Católica de Chile, Casilla 306, Santiago 22, Chile \\ ${ }^{6}$ Centre de Recherche Astrophysique de Lyon (CRAL), Observatoire de Lyon, 9 avenue Charles André, F-69230 Saint-Genis-Laval, France \\ ${ }^{7}$ Space Telescope Science Institute, 3700 San Martin Drive, Baltimore, MD 21218, USA \\ ${ }^{8}$ Department of Physics \& Astronomy, Stony Brook University, Stony Brook, NY 11794-3800, USA \\ ${ }^{9}$ Spitzer Science Center, Pasadena, CA 91125 , USA \\ ${ }^{10}$ Eureka Scientific, Inc., 2452 Delmer Street Suite 100 Oakland, CA 94602-3017, USA \\ ${ }^{11}$ Department of Astronomy, The University of Texas at Austin, 2515 Speedway Boulevard Stop C1400, Austin, TX 78712, USA \\ ${ }^{12}$ Department of Physics and Astronomy, Howard university, 2355 6th Street NW, Washington, DC 20059, USA \\ ${ }^{13}$ Department of Astronomy, University of Maryland, College Park, MD 20742, USA \\ ${ }^{14}$ REU at the National Radio Astronomy Observatory, 520 Edgemont Road, Charlottesville, VA 22903, USA \\ Received 2016 October 17; revised 2017 May 2; accepted 2017 May 8; published 2017 July 6
}

\begin{abstract}
We present the results of a Hubble Space Telescope ACS/HRC FUV, ACS/WFC optical study into the cluster populations of a sample of 22 Luminous Infrared Galaxies in the Great Observatories All-Sky LIRG Survey. Through integrated broadband photometry, we have derived ages and masses for a total of 484 star clusters contained within these systems. This allows us to examine the properties of star clusters found in the extreme environments of LIRGs relative to lower luminosity star-forming galaxies in the local universe. We find that by adopting a Bruzual \& Charlot simple stellar population model and Salpeter initial mass function, the age distribution of the clusters declines as $d N / d \tau=\tau^{-0.9+/-0.3}$, consistent with the age distribution derived for the Antennae Galaxies, and interpreted as evidence for rapid cluster disruption occurring in the strong tidal fields of merging galaxies. The large number of $10^{6} M_{\odot}$ young clusters identified in the sample also suggests that LIRGs are capable of producing more high-mass clusters than what is observed to date in any lower luminosity star-forming galaxy in the local universe. The observed cluster mass distribution of $d N / d M=M^{-1.95+/-0.11}$ is consistent with the canonical -2 power law used to describe the underlying initial cluster mass function (ICMF) for a wide range of galactic environments. We interpret this as evidence against mass-dependent cluster disruption, which would flatten the observed CMF relative to the underlying ICMF distribution.
\end{abstract}

Key words: galaxies: active - galaxies: individual (NGC 3256, NGC 3690, NGC 1614) - galaxies: interactions infrared: galaxies

\section{Introduction}

Galaxies with high infrared (IR) luminosities, e.g., luminous infrared galaxies (LIRGs: $L_{\mathrm{IR}}[8-1000 \mu \mathrm{m}]>10^{11.0} L_{\odot}$ ), are rare in the local universe, yet they are a cosmologically important class of objects because they dominate the infrared luminosity density at redshifts $z=1-2$ (Magnelli et al. 2011). Their high bolometric luminosities emanate from energetic star formation (SF) regions, and sometimes active galactic nuclei (AGNs), which are primarily triggered by interactions and mergers of gas-rich galaxies (e.g., Sanders \& Mirabel 1996). Further, the complex structure of these dynamically evolving systems and the presence of both dust-obscured and unobscured activity necessitates the need for high-resolution observations that sample as much of the electromagnetic spectrum as possible to best identify and reconstruct the distribution and luminosity of star formation and AGN-related phenomena, and to probe the connection between merger stage and the observed activity. Understandably, the ultraviolet (UV) properties of these very IR-luminous galaxies have received far less scrutiny. However, the small fraction of the UV radiation from super star clusters, AGNs, and diffuse stellar emission that escapes can nonetheless make LIRGs powerful sources of UV radiation (e.g., Evans et al. 2008; Armus et al. 2009; Howell et al. 2010; Inami et al. 2010).

Of interest to the present study of LIRGs are the luminous star clusters, which track basic information regarding the formation and fate of star formation in a variety of different environments. The Hubble Space Telescope (HST) has been instrumental in the detection of numerous star clusters ( $\gtrsim 1000)$ in gas-rich mergers (e.g., NGC 3256: Zepf et al. 1999; NGC 4038/9: Whitmore \& Schweizer 1995, Whitmore et al. 1999) and recent merger remnants (e.g., NGC 3921: Schweizer et al. 1996; NGC 7252: Miller et al. 1997; Schweizer \& Seitzer 1998; NGC 3610: Whitmore et al. 1997). The presence of young $(\lesssim 10 \mathrm{Myr})$ and intermediate age (100-500 Myr) star cluster populations in late-stage mergers such as the Antennae galaxies (NGC 4038/4039; Whitmore et al. 1999), Arp 220 (Wilson et al. 2006), and the Mice galaxies (NGC $4676 \mathrm{~A} / \mathrm{B}$; Chien et al. 2007) is consistent with the description of these galaxies as experiencing powerful starbursts triggered by the interaction and merger of pairs of gas-rich galaxies. However, 
optical studies of other late-stage mergers such as NGC 6240 (Pasquali et al. 2003) and NGC 7673 (Homeier et al. 2002) reveal only young star clusters, indicating that older star clusters, which would have formed earlier on in the merger, are either undetected or rare. In contrast, the lack of young star clusters in the tidal tails of NGC 520 and NGC 2623 (Mulia et al. 2015) relative to what is observed for NGC 3256 (Trancho et al. 2007) suggests that the remaining reservoirs of predominately neutral hydrogen (H I) gas in the tails cannot always form new clusters.

Many studies have been devoted to understanding the longterm stability of the youngest clusters in mergers (e.g., Whitmore et al. 2007; Fall et al. 2009; Kruijssen et al. 2011). It appears that only those that survive the disruption processes and are still dense and gravitationally bound are likely to become the globular clusters (GCs) we observe today (Zhang $\&$ Fall 1999). The relative contributions from various cluster disruption mechanisms such as infant mortality (Fall et al. 2005; Chandar et al. 2010), two-body relaxation (Tutukov 1978; Fall et al. 2009), and tidal shocks (Gnedin \& Ostriker 1997) as a function of galactic environment continues to be the subject of much work. Infant mortality or rapid disruption is caused by mass loss during the early gas expulsion phase of cluster evolution and is expected to work on timescales of $\leqslant 10$ Myr. In contrast, disruption from large-scale shocks is expected to be important over roughly $10^{8}$ year timescales, and two-body relaxation will cause disruption on even longer timescales (on the order of a Hubble time). Ultimately, the manner in which these young massive clusters (YMCs) evolve is crucial to connecting them to present-day globular clusters. If YMCs are indeed local analogues to present-day GCs, then by understanding their formation and evolution, it is possible to gain insight into the formation of the earliest most massive clusters in the universe (Kruijssen 2014).

In addition to understanding the fate of clusters, it is important to understand to what degree their environment affects where and how they form, as well as what their collective properties are-e.g., the distribution of massive clusters (Initial Cluster Mass Function: ICMF) and the efficiency with which bound star clusters form (Larsen \& Richtler 2000; Bastian 2008a). Although the low-mass end of the ICMF appears to be universal (de Grijs et al. 2003; Fall \& Chandar 2012), the formation conditions of the highest-mass clusters are still subject to debate.

One idea is that the formation mechanism of the most massive clusters is independent of environment (Whitmore et al. 2007; Chandar et al. 2015), and thus the total number and maximum cluster mass scale linearly with the star formation rate of the galaxy (Hunter et al. 2003; Whitmore et al. 2010; Vavilkin 2011). Alternatively, the formation of the most massive clusters may require special physical conditions, such as high ambient pressure or enhanced gas densities. Kruijssen (2012) predict that the formation of bound stellar clusters takes place in the highest-density peaks of the ISM. Therefore, YMCs should form more efficiently at high gas pressures (and hence gas surface densities), because these conditions lead to higher density peaks. This leads to a nonlinear scaling of the maximum cluster observed and the star formation rate surface density $\left(\Sigma_{\mathrm{SFR}}\right)$ of the galaxy.

To really quantify the role of galactic environment in shaping massive cluster formation and destruction, we need to study the properties of star clusters in a statistically larger sample of

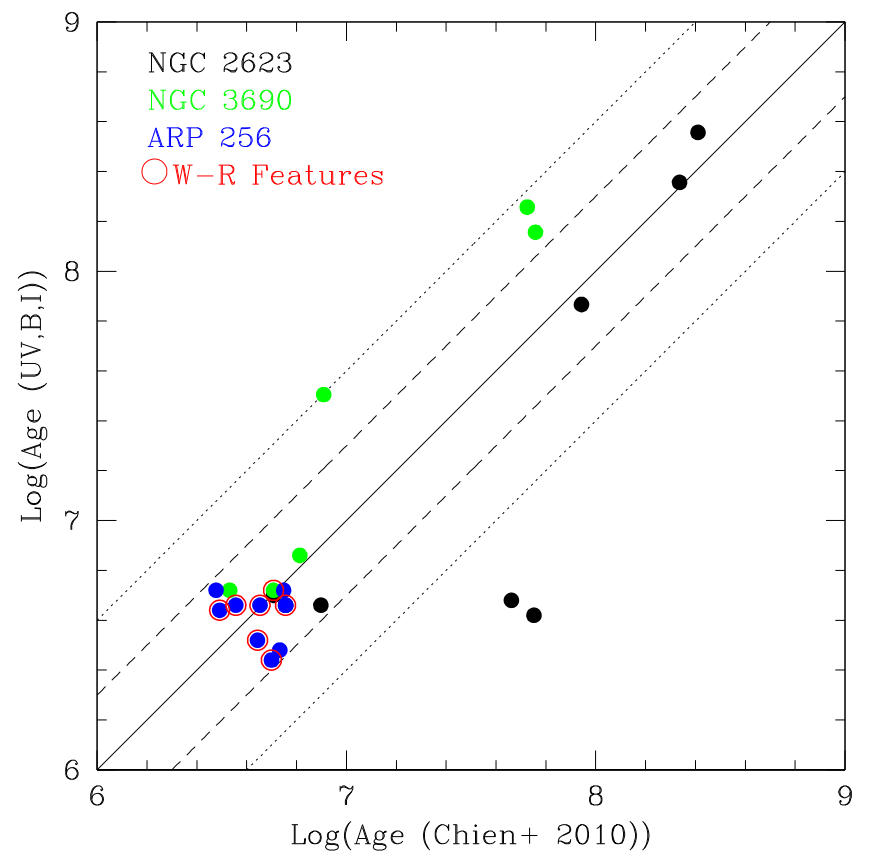

Figure 1. Comparison between the spectroscopically derived ages from Chien (2010) and our UV, $B$, and $I$ broadband age estimates for NGC 2623, NGC $3690 \mathrm{E} / \mathrm{W}$, and ARP $256 \mathrm{~N} / \mathrm{S}$. The red circles denote star clusters that have Wolf-Rayet spectral features as identified in Chien (2010). The solid line represents the 1:1 correlation, whereas the dashed and dotted lines are within 0.3 and 0.6 dex of the $1: 1$ correlation.

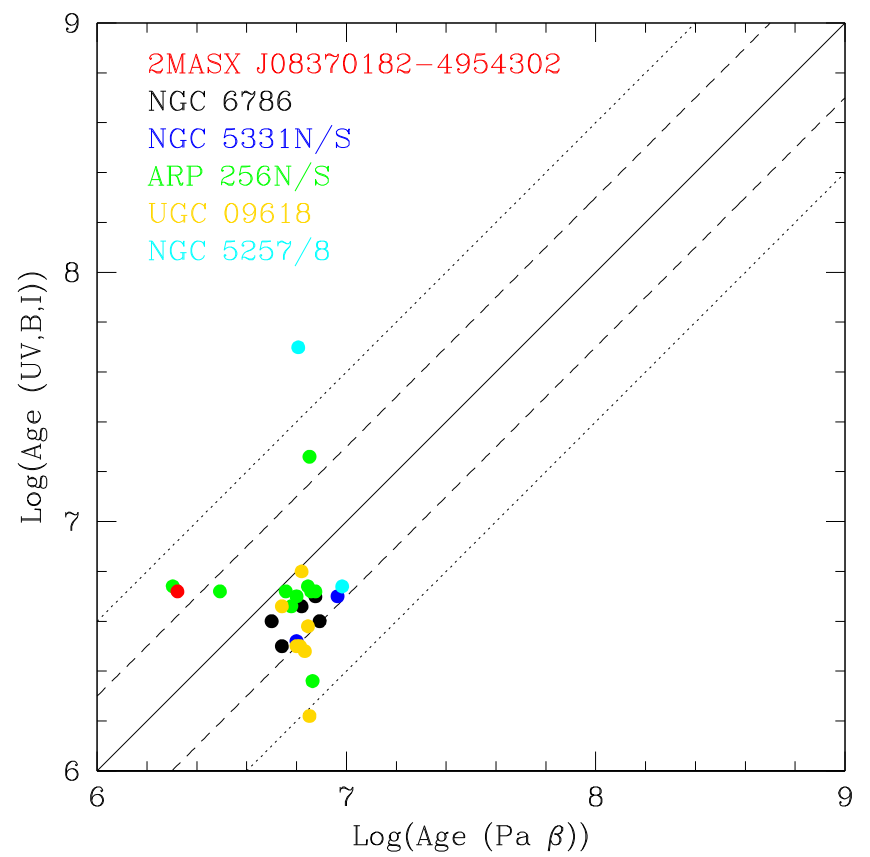

Figure 2. One-to-one comparison of cluster ages derived using our $\mathrm{UV}, B$, and $I$ photometry and the equivalent width of the $\mathrm{Pa} \beta$ emission line associated with the cluster centroid from K. L. Larson et al. (2017, in preparation). The solid line represents the 1:1 correlation, whereas the dashed and dotted lines are within 0.3 and 0.6 dex of the $1: 1$ correlation.

LIRGs, which represent the most extreme star-forming systems observed in the local universe. The Great Observatories All-Sky LIRG Survey (GOALS) is a multiwavelength imaging and spectroscopic study of a complete flux density-limited $\left(S_{60 \mu \mathrm{m}}>5.24 \mathrm{Jy}\right)$ sample of the 202 LIRGs in the IRAS Revised Bright Galaxy Sample (RBGS, Sanders et al. 2003; 


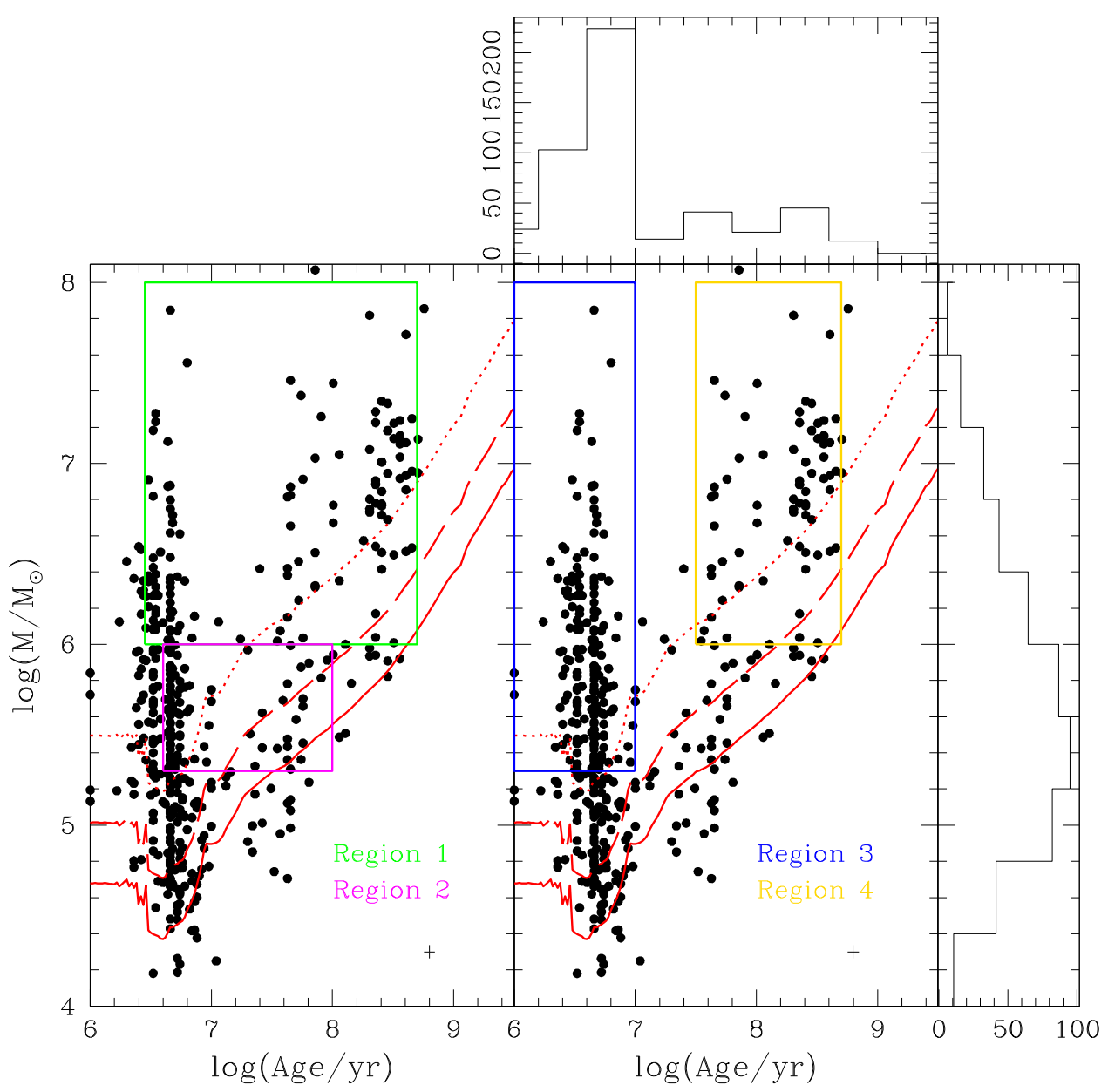

Figure 3. Mass and age distribution of all 484 clusters found in the 27 galaxies. The solid, dashed, and dotted red curves represent mass-age tracks produced from the BC03 model with an input of $M_{B}=-11.26, M_{B}=-12.07$, and $M_{B}=-13.31$ for the $50 \%, 75 \%$, and $100 \%$ completeness limits, respectively. The green and purple boxes in the left panel represent Regions 1 and 2, respectively, and are used for the two mass-age cuts applied when analyzing the cluster age distribution. The blue and gold boxes in the middle panel represent Regions 3 and 4, respectively, and are used for the two mass-age cuts applied when analyzing the cluster mass distribution. The histograms show the distribution of cluster ages and masses for the full sample. The cross on the bottom right of each panel represents the median errors in cluster age and mass bootstrapped from our model.

GOALS, Armus et al. 2009). The proximity, size, and completeness of the sample, combined with broad wavelength coverage, make GOALS the definitive sample for studying star clusters in local, luminous star-forming galaxies. The present study makes use of HST UV and optical images from GOALS to estimate the cluster age distribution, the cluster mass function, and the cluster formation efficiency in a sample of 22 LIRGs (Table 1).

The paper is organized as follows. In Section 2, the sample selection is summarized. In Section 3, the observations and data reduction are described, as well as our method for identifying clusters. In Section 4, the manner in which the cluster ages are estimated is described. In Section 5, the age distribution, the mass function, and the cluster efficiency are discussed within the context of lower luminosity star-forming galaxies. Section 6 is a summary of the results.

Throughout this paper, we adopt a WMAP cosmology of $H_{0}=70 \mathrm{~km} \mathrm{~s}^{-1} \mathrm{Mpc}^{-1}, \Omega_{\text {matter }}=0.28$, and $\Omega_{\Lambda}=0.72$ (e.g., see Armus et al. 2009).

\section{Sample Selection}

Within GOALS, there are HST $B$ - and $I$-band observations of all 88 LIRGs with $L_{\mathrm{IR}} \geqslant 10^{11.4} L_{\odot}$. Of those, we select the 22
LIRGs observed to have greater than $100 \mathrm{~B}$-band luminous clusters $\left(m_{\mathrm{B}} \sim 21-23 \mathrm{mag}\right)$ within the central $30^{\prime \prime} \times 30^{\prime \prime}$ of the galaxy (i.e., a limit imposed by our far-UV imaging field of view-see below). In total, we observed $9131 \mathrm{~B}$-band luminous star clusters from galaxies in the sample.

\section{Observations, Data Reduction, and Cluster Selection}

The HST B-band $(\mathrm{F} 435 \mathrm{~W})$ and $I$-band $(\mathrm{F} 814 \mathrm{~W})$ images were obtained with the Wide Field Camera (WFC) on the Advanced Camera for Surveys (ACS) during the period 2005 August to 2007 January (PI: A. Evans; PID 10592). In all but a few cases, the wide field of view of the WFC $\left(202^{\prime \prime} \times 202^{\prime \prime}\right)$ enabled the full extent of each LIRG to be observed. Each galaxy was observed in both filters per orbit, with two and three dithered exposures in ACCUM mode in the F814W filter and F435W filters, respectively. The approximate integration times for each filter were 21 minutes in F435W and 12 minutes in F814W. The ACS data were reduced with the Multidrizzle software included in IRAF/STSDAS provided by STScI to identify and reject cosmic rays and bad pixels, remove geometric distortion, and combine the images into mosaics. Because of the limited number of dithers, additional cosmic-ray rejection routines 
Table 1

Properties of the 27 GOALS Galaxies in the Sample

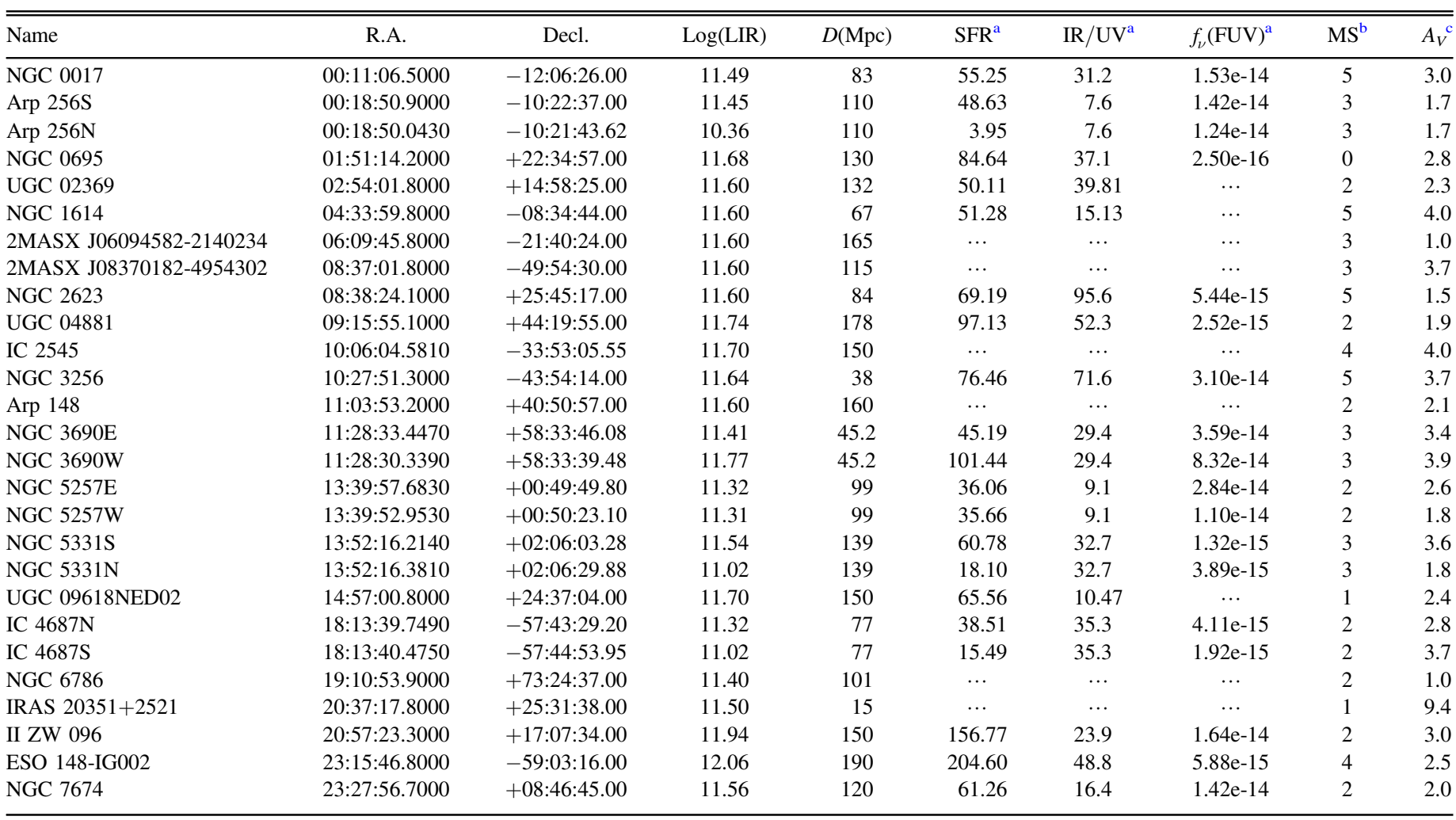

Notes.

${ }^{\text {a }}$ SFRs calculated using IR+UV data taken from Howell et al. (2010) and U et al. (2013).

${ }^{\mathrm{b}}$ Merger stages taken from Haan et al. (2013) and Stierwalt et al. (2013).

${ }^{\mathrm{c}}$ The maximum $A_{V}$ adopted for each galaxy taken from the literature. See the Appendix for more details.

Table 2

Derived Age and Mass Function Slopes

\begin{tabular}{lccccccc}
\hline \hline Name & $\gamma_{0.02}$ & $\sigma_{\gamma}$ & $\gamma_{0.008}$ & $\sigma_{\gamma}$ & $\beta_{0.02}$ & $\sigma_{\beta}$ & $\beta_{0.008}$ \\
\hline NGC 1614 & -0.96 & 0.18 & -1.16 & 0.17 & -1.35 & 0.23 & -1.60 \\
NGC 7674 & -1.67 & 0.46 & -0.78 & 0.28 & -1.15 & 0.12 & -1.32 \\
NGC 3690E & -0.62 & 0.54 & -1.01 & 0.44 & -1.44 & 0.14 & -1.31 \\
NGC 3690W & -1.26 & 0.12 & -1.24 & 0.14 & -1.92 & 0.24 & -1.45 \\
Arp 148 & -0.87 & 0.38 & -1.38 & 0.69 & -1.44 & 0.17 & -1.8 \\
IRAS 20351+2521 & -1.19 & 0.11 & -1.27 & 0.10 & -1.60 & 0.52 & -1.12 \\
NGC 6786 & -1.29 & 0.18 & -1.17 & 0.26 & -1.40 & 0.12 & -1.58 \\
UGC 09618NED02 & -1.18 & 0.23 & -1.42 & 0.12 & -2.13 & 0.47 & -1.52 \\
\hline
\end{tabular}

were run on each image prior to drizzling (see Kim et al. 2013 for a detailed description).

The HST far-UV (F140LP) and optical images in the sample were obtained with the Solar Blind Channel (SBC) on the Advanced Camera for Surveys (ACS) during the period 2008 April-2009 August (PID 11196; PI: A. Evans). The field of view of the SBC is $\sim 30^{\prime \prime} \times 30^{\prime \prime}$-this placed a limit on the area within each LIRG over which the clusters could be analyzed. The data were taken in the ACCUM mode using the PARALLELOGRAM four-position dither pattern for a total integration time per galaxy of 40-45 minutes. We further reduced the SBC data with the Multidrizzle software included in $I R A F / S T S D A S$ provided by STScI to identify and reject cosmic rays and bad pixels, remove geometric distortion, and combine the images into mosaics.

Before an automated routine for cluster identification could be applied to the images, contamination from foreground stars and distant background galaxies outside of the area of each image subtended by the LIRG (i.e., the "sky" area) had to be minimized. Masks of each image were made by first creating a median-smoothed version of the $\mathrm{F} 435 \mathrm{~W}$ and F814W images. The effect of this filtering is to minimize structures in the sky region with spatial extents significantly smaller than the filter size (i.e., faint stars and distant background galaxies). The backgrounds, containing low pixel values, were then set to zero, while the high pixels 


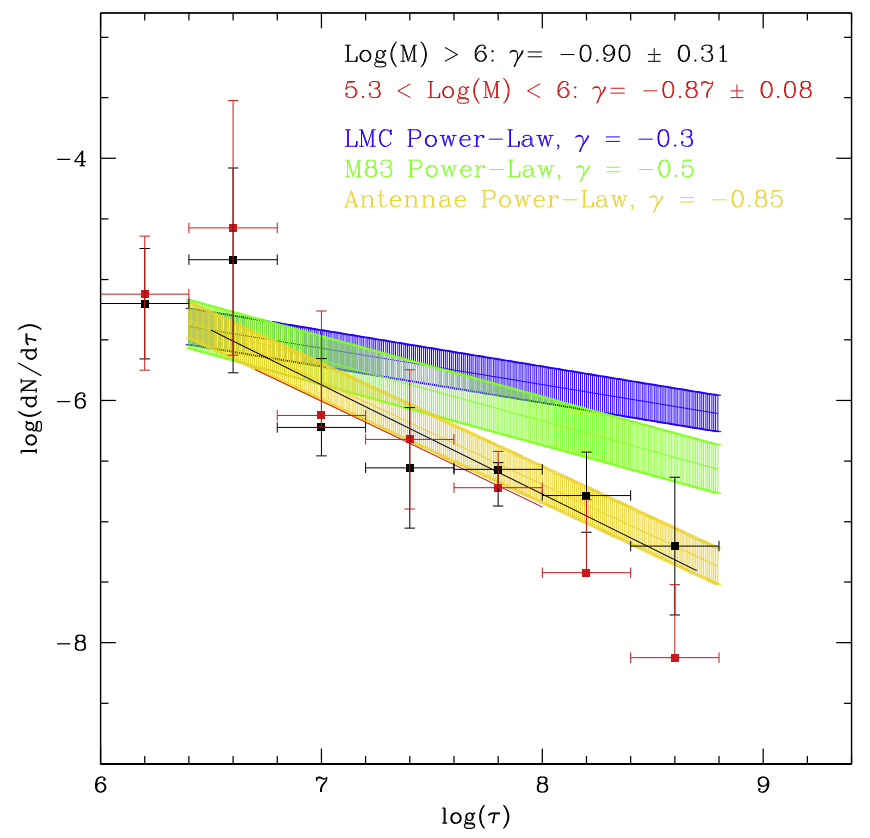

Figure 4. Stacked age distribution functions for all 27 galaxies. We have broken our age distribution up into the two age-mass ranges described in Equations (1) and (2), and shown as Regions 1 and 2 in the left panel of Figure 3 . The red and black lines represent the weighted linear least-squares fits to the data. The blue, green, and yellow age functions of the LMC, M83, and the Antennae, respectively, are taken from Adamo \& Bastian (2015), and are normalized to the total number of clusters in our sample to best compare the slope for each galaxy.

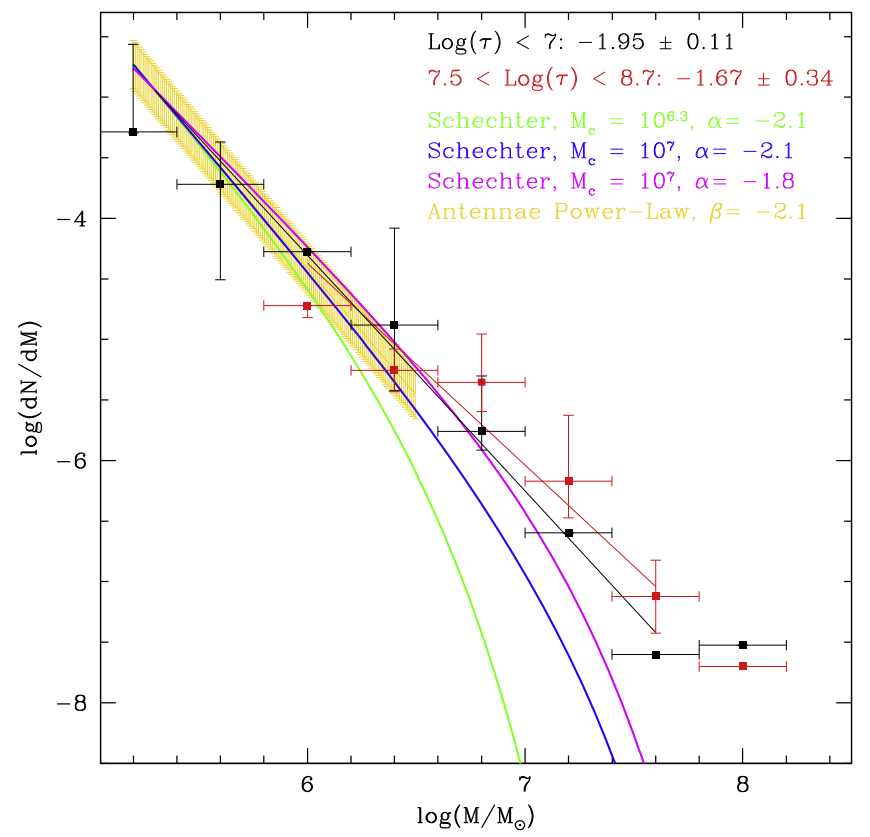

Figure 5. Stacked mass distribution functions for all 27 galaxies. We have separated our mass distribution into two mass-age ranges described in Equations (3) and (4), and shown as Regions 3 and 4 in the right panel of Figure 3 . These cuts allow us to test the effects of our completeness limits and the mass dependence of cluster disruption in the sample. The red and black lines represent the by weighted linear least-squares fits to the data. The yellow error bars represent the mass function of the Antennae taken Whitmore et al. (2010), and is normalized to the total number of clusters in our sample. The green, magenta, and blue lines represent three different analytic Schechter function fits to the empirical distribution.
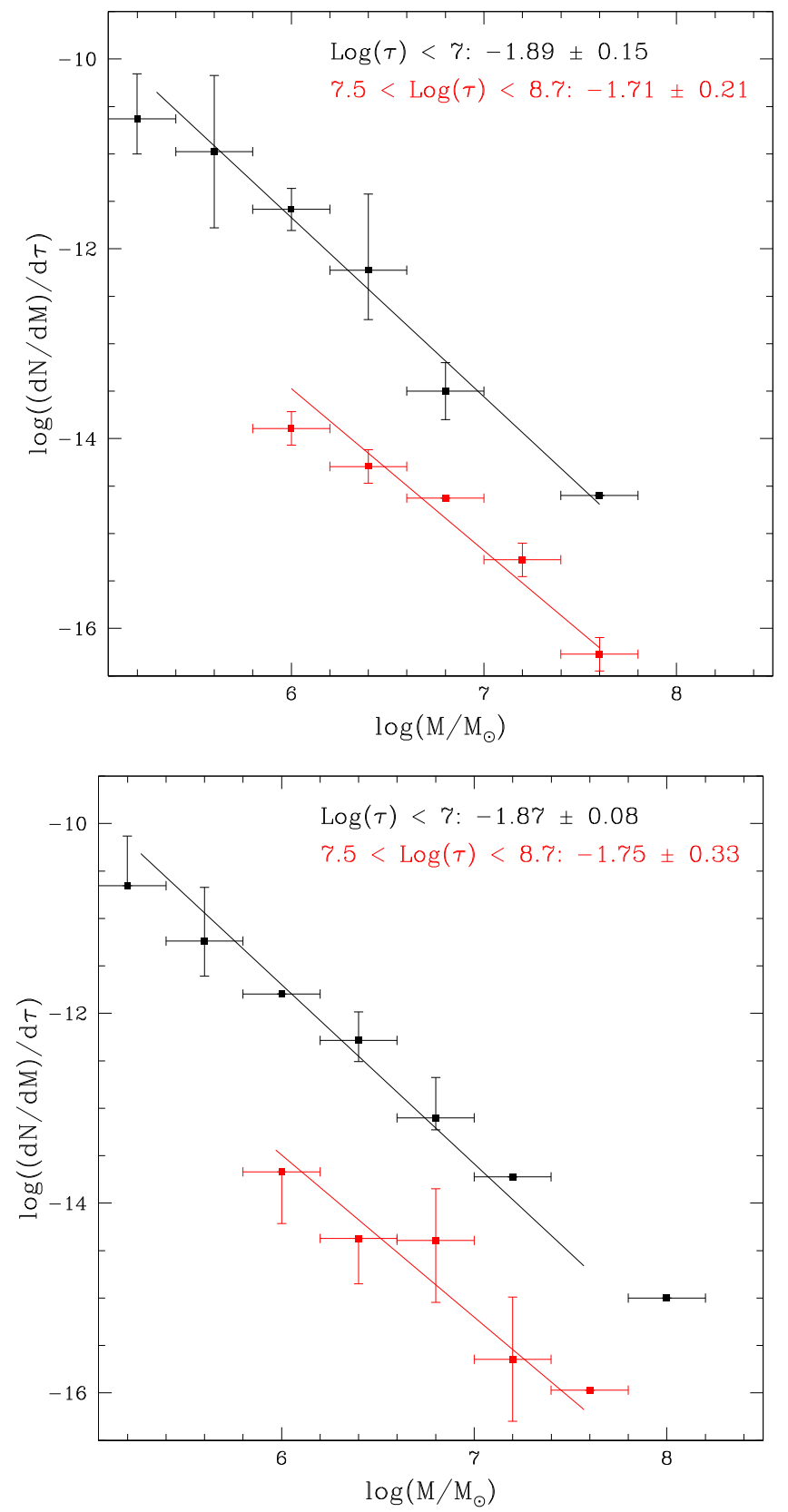

Figure 6. Stacked age-normalized mass distribution functions for the 11 galaxies with a merger class of $0-2$ (top plot) and the 8 galaxies with a merger class 3-4 (bottom plot) identifying them as early-stage and mid-stage mergers, respectively. The total number of clusters in each class is 260 and 154 for preand mid-stage mergers, respectively. We have broken up our mass distribution into the same age ranges as in Figure 5.

corresponding to the LIRG were set to one. Finally, pixels associated with any bright stars in the image were set to zero. The original reduced image was then multiplied by the final mask of the galaxy to set the regions outside of the galaxy equal to zero.

Star clusters in all three bands were selected using the program SExtractor (Bertin \& Arnouts 1996). The identification of clusters and the extraction of photometry is complicated by the non-uniform surface brightness of the underlying galaxy. To estimate and subtract the underlying galaxy, Source Extractor iteratively computes the median and standard 
deviation of the pixels within a mesh of $n \times n$ pixels. During each iteration, outlier pixels are discarded until all of the pixels within each mesh are within $3 \sigma$ of the median value. Several mesh sizes were tested, and for each mesh the photometry of several of the clusters was separately computed via the IPAC image display and analysis program Skyview and compared to values estimated from the original image (Skyview allows users to manually size and place apertures on clusters, and it allows for local background around the aperture to be subtracted). The mesh sizes varied between 9 and 14 pixels, and overall did an efficient job of removing the underlying galaxy and minimizing the creation of negative value holes surrounding clusters created through oversubtraction of the local background. Cluster photometry across all backgroundsubtracted images was then calculated using the IDL package APER (originally modified from DAOPHOT). We used an aperture of radius 6.0 pixels for the HRC images and 3.0 pixels for the WFC images ( $=0$ !" 15 in both cases). An annulus with a radius of 4 pixels and a thickness of 5 pixels was used to measure the local background in the WFC images; the radii and thickness of the annulus was adjusted accordingly for the SBC images. Aperture corrections were calculated based on the flux calibrations of unresolved sources by Sirianni et al. (2005). We corrected the photometry for foreground Galactic extinction using the Schlafly \& Finkbeiner (2011) dust model combined with the empirical reddening law of Fitzpatrick (1999) available through the NASA Extragalactic Database (NED).

In the process of doing the photometry, we filtered out all sources that had a signal-to-noise ratio $\mathrm{S} / \mathrm{N}<5$ and that were not visible in all three filters. This left us with a total of 1186 cluster candidates identified in the sample. We then used ISHAPE (Larsen 1999) to measure the FWHM values for all remaining sources in all three wavelengths; this was done in order to separate stars and background galaxies from clusters. ISHAPE measures FWHMs by deconvolving the HST instrumental point-spread function with a King profile, then performing a $\chi^{2}$ calculation to test the goodness of fit to each individual cluster (King 1966). ISHAPE iterates through different values for the effective radius until a minimum $\chi^{2}$ is found. Similar to the approach in Mulia et al. (2015), we find that a conservative cut of 2 pixel FWHM effectively removes extended sources in both the nearest and farthest galaxies in the sample. Additionally, we made a cut of $M_{B} \leqslant-9.5 \mathrm{mag}$, corresponding to the Humphreys \& Davidson (1979) limit, where we might expect contamination of the cluster sample from single bright yellow supergiants in the Milky Way. This was shown in Whitmore et al. (2010) to be an effective way to remove foreground stars by their luminosity alone. A total of 665 clusters across all 22 LIRGs (27 nuclei) meet the above criteria.

One remaining concern with this approach was that at the average distance of the galaxies in our sample $(115 \mathrm{Mpc})$, our size estimates would not correspond to physically relevant values for individual clusters. Indeed, a 2 pixel FWHM at the resolution of WFC gives an average cluster size of $R_{\text {eff }} \sim 24$ pc. For the most nearby galaxies in the sample, we derive consistent results with the established cluster size in the Antennae of $R_{\text {eff }} \sim 5-10$ pc (Anders et al. 2007). However, for the most distant galaxies in the sample, our size estimates are nearly three times larger $(\sim 37 \mathrm{pc})$, which is an effect we must take into consideration when interpreting our results (see
Section 5). Importantly, the measured cluster sizes are all still well below the average size of an entire cluster complex or OB association ( $R_{\text {eff }} \sim 100-200$ pc: Bastian et al. 2006), where the application of simple stellar population (SSP) models would be questionable.

\section{Age-dating Clusters}

\subsection{Model Fitting}

For each cluster in each galaxy, the measured colors were compared with the evolutionary tracks from GALAXEV (version 2003), a library of evolutionary stellar population synthesis models that were computed using the isochrone synthesis code of Bruzual \& Charlot (2003), hereafter referred to as BC03. This code computes the spectral evolution of a stellar population based on a stellar evolution prescription (Girardi et al. 2002) and a library of observed stellar spectra. The output of the model SED was multiplied by the ACS F435W, F814W, and SBC 140LP filter response functions in order to obtain magnitudes and colors in these filters. We first estimate the age and the extinction $A_{V}$ by performing a $\chi^{2}$ fit assuming an instantaneous burst simple stellar population (SSP), a Salpeter IMF (Salpeter 1955), and both solar and subsolar metallicities as suggested for LIRGs by Kewley et al. (2010). We also apply a Calzetti extinction law of the form $k(\lambda)=A(\lambda) / E(B-V)_{*}=$ $a+b / \lambda+c / \lambda^{2}+d / \lambda^{3}$, where $a, b, c$, and $d$ are constants in a given wavelength range, and $A(\lambda)$ is the attenuation in magnitudes. The total attenuation of the stellar continuum, $R_{V}=A(V) / E(B-V)_{*}=4.05 \pm 0.8$, is calibrated specifically for starburst galaxies and differs from the typical Milky Way value of $R_{V} \sim 3.1$ (Calzetti et al. 2000). It has been shown empirically that clusters and $\mathrm{HII}$ regions are more heavily attenuated than the underlying stellar continuum, due to the fact that these objects are often found near dusty regions of ongoing star formation (Calzetti et al. 1994). From galaxy to galaxy, there can be considerable variations in the detailed dust distributions, but Calzetti et al. (2000) points out that in all the cases they studied, the empirical law recovers the total dust optical depth of UV-bright starburst galaxies within a factor of two.

It is worth noting here that a major concern in estimating cluster ages is the effect of stochasticity, which affects clusters with low masses. Such clusters have too low a mass to adequately produce a sufficient of number of stars in all mass ranges, and thus any age-dating prescription making use of a standard IMF fails to predict the correct cluster age. Given the distance of the LIRGs in the sample and thus the brightness of the clusters detected by our HST observations, the detected clusters are unlikely to have low masses. Indeed, stochastic fluctuations are relatively minor for clusters with masses greater than $10^{4} M_{\odot}$ (Fouesneau et al. 2012), which in our case is the lower limit of the clusters we can observe.

Another factor affecting the age estimates is the metallicity. LIRGs are known to have gas-phase metallicities within 0.2 dex of solar in $12+\log [\mathrm{O} / \mathrm{H}]$ (Relaño et al. 2007; Rupke et al. 2008). Thus, we consider both a solar $(z=0.02)$ and subsolar $(z=0.008)$ BC03 model for each galaxy. Rich et al. (2012) also find that the metallicity gradients in LIRGs are flattened by the merging process, allowing us to parameterize the metallicity of clusters with a single value for each galaxy.

The mass of each cluster is estimated from the observed $B$ band extinction-corrected luminosity and the mass-to-light 
ratios $\left(M\left(M_{\odot}\right)=L_{B} \times(M / L)\right)$ predicted by the unextincted model at the fitted age. The models assume that the stellar IMF for each cluster is fully sampled. The largest contribution to the uncertainty in the mass estimates is the uncertainties in the estimated ages, which are typically on the order of $0.3 \mathrm{dex}$ in $\log (\tau)$. These translate to similar uncertainties of 0.3 and 2 in $\log (M)$ and $M$, respectively. The derived masses of the clusters depend on the IMF assumed in the stellar population models. For example, if a Chabrier IMF is adopted, the estimated mass of each cluster would decrease by a near constant $40 \%$ (although the shape of the mass function would not change). The average fractional uncertainty in the distances of each galaxy taken from NED are $\sim 7 \%$. This would introduce uncertainties in the cluster mass estimates of roughly $13 \%$, which is less than the error contribution from our cluster agefitting procedure.

The age and mass estimations using color-color diagrams together with evolutionary tracks suffer from age-reddening degeneracy. As pointed out in Maoz et al. (2001), the use of a UV filter when examining the colors of star clusters does help to avoid the issue of "backtracking," whereby the reddening shifts the models in a direction nearly parallel to the aging direction. However, a cluster that appears red in the FUV- $B$, $B-I$ color space can still be either very old, or young and heavily obscured by dust. In particular, young star clusters are assumed to be embedded in dust that is present in the starforming region. Despite the fact that a fraction of the dust can be cleared away from young star-forming regions in as little as a few Myr (Larsen 2010), $0.5 \leqslant A_{V} \leqslant 2.5$ mag extinction has been reported for $4 \mathrm{Myr}$ old clusters in nearby, lower luminosity galaxies (Whitmore \& Zhang 2002; Reines et al. 2008). Since our analysis involves the use of three filters, we cannot break this degeneracy with our photometry alone. Thus, the ages of clusters in our LIRG sample are solved for by creating a suite of SSPs within the FUV- $B, B-I$ color space, incrementing by 0.1 in $A_{V}$ as input to the extinction law, then solving for the age reddening of each cluster based on the best $\chi^{2}$ fit to an individual model within the suite. Further, it is important to note that because FUV light can accurately trace the ages of star clusters over two orders of magnitude (Meurer et al. 1995), our analysis of cluster ages is not biased by the requirement to detect a cluster in the F140LP SBC filter.

In order to better refine the age-reddening estimates for each cluster, two additional constraints were applied: first, we required that the extinction of any given cluster could not exceed estimates for the $A_{V}$ of its host galaxy taken from the literature. Considering the fact that our F140LP cluster detections often span the entire SBC field of view, the average galaxy $A_{V}$ is a good proxy for the amount of reddening one would expect each cluster could have before we are unable to detect it. It is important to note that only $5 \%$ of clusters in the final sample have extinctions that are equal to the maximum allowed for their host galaxy based on our fits, meaning that our choice of $A_{V}$ is not systematically biasing our final derived values. This constraint additionally prevents our model from obtaining cluster properties with arbitrarily high extinctions and therefore cluster masses, which exceed what is possible for bound stellar clusters so far observed in extragalactic systems (Maraston et al. 2004).

Second, we constructed $B-I$ color images in order get a visual clue of where the projected dust lanes are in each galaxy. The reasoning is that by making a manual assessment of each image, we can distinguish globular clusters, which have much redder colors and are often found in uncrowded regions away from sites of recent star formation (e.g., see Whitmore et al. 2014). One complicating factor is that a YMC that forms behind a projected dust lane can appear to have color similar to these old GCs. By overlaying the cluster centroids, we identified which clusters had no obvious dust lanes in a surrounding annulus of 4-9 pixels. These clusters are therefore more likely to be young and extincted as opposed to relatively old and dust-free clusters. The results can be seen in the falsecolor images shown in the Appendix. In total, only $10 \%$ of the clusters modeled had ages that differ by 0.6 dex (roughly twice the expected uncertainty) when including or excluding the additional dust-lane constraints. Whitmore et al. (2014) used this additional constraint when looking at the cluster populations of 20 star-forming galaxies in the local universe, and found it to be effective regardless of the detailed galaxy morphologies seen in the color images.

We consider here how these constraints can be understood based on the $F 435 W-F 814 W$ value of each cluster: clusters designated with $(F 435 W-F 814 W)<0.51$ mag can be reliably age-dated as being younger than $7 \mathrm{Myr}$, because the old-age track of the model never reaches that part of the parameter space. Clusters with $(F 435 W-F 814 W)=0.51-1.0$ mag have a wide range of possible ages (7-500 Myr), but if the cluster resides in a dustier region of the galaxy, then it is either an unreddened to moderately reddened old cluster or a young, heavily reddened cluster. This color bin covers the widest range of cluster ages and therefore contains the largest number of star clusters. Finally, star clusters with $(F 435 W-F 814 W)=1.0-1.5 \mathrm{mag}$ that do not reside in a more heavily extincted region of the galaxy are old, with ages between $500 \mathrm{Myr}$ and $1 \mathrm{Gyr}$. The ages of clusters in these last two regions that lie in and around dust lanes are the ones most affected by our above criteria for solving the age-reddening degeneracy. Clusters with $(F 435 W-F 814 W)>1.5$ have ages older than $1 \mathrm{Gyr}$ assuming reasonable values for the internal extinction within the galaxy.

By examining the distributions of internal visual extinction and age for each cluster derived from the model, we see that nearly one-third of all young clusters in the sample have a relatively small dust correction $\left(A_{V} \leqslant 1\right)$, and nearly $80 \%$ of all young clusters have an $A_{V} \leqslant 2$ correction. Thus, the majority of all clusters in the sample need only a relatively modest dust correction, compared to the global average of galaxies, to properly derive young ages.

\subsection{Consistency Checks}

\subsubsection{Comparison with Direct SED Fitting}

In order to account for the effect our chosen filter set has on the derived cluster properties as described above, we compare the results of anchoring each color to the F435W measurement, with the results from fitting the three broadband photometric measurements (F140LP, F435W, and F814W) simultaneously, as was similarly done in Maoz et al. (2001), and shown to be an effective way to further improve our ability to separate the effects of age and extinction. To perform this full "SED-based" fitting, we use the same galaxy evolution code, extinction model (minus the additional dust-lane constraints in both cases), IMF, and metallicity. From our sample of 665 clusters, we further remove from the final analysis any clusters for which the method described in Section 4.1 and this SED-fitting 
method do not produce ages that agree within 0.6 dex of each other. These clusters are almost always ones for which there is nearly equal probability of the cluster being young and highly extincted, or old and less heavily extincted. These highly degenerate cases are therefore removed due to their uncertain contribution to the overall shape of the age and mass distributions to be derived. This leaves us with a final sample of 484 ( $\sim 83 \%$ of verified clusters) clusters that have age and mass estimates independent of the fitting method chosen for deriving cluster properties. We also note that of the original 67 clusters that provide inconsistent age results in our own dustlane versus no-dust-lane analysis, $48(\sim 83 \%)$ are kept when comparing to the results of the full SED fit. This again shows that our additional dust-lane constraints did not systematically bias the estimation of cluster ages.

\subsubsection{Comparisons with Spectroscopic-derived Ages}

Chien (2010) measured Balmer line-derived cluster ages for a sample of GOALS LIRGs. Three of the systems in their sample overlap with our present study (NGC 2623, Arp 256, and Arp 299). Figure 1 is a comparison of our photometrically derived ages and the Balmer line-derived ages. Approximately $77 \%$ (17 of 22) of the clusters have ages that agree to within \pm 0.3 dex, and $91 \%$ (20 of 22 ) have ages that agree to within \pm 0.6 dex. This means that the majority of our three-band cluster ages agree with the spectroscopic ages within the uncertainty of the $\mathrm{BC} 03$ models. Further, it is important to note that we derive young ages for all seven of the star clusters in our sample with identified Wolf-Rayet spectral features from Chien (2010). Wolf-Rayet features are very sensitive probes of young cluster ages since they only exist for clusters with ages of 3-7 Myr (Leitherer et al. 1999; Chien 2010).

It is potentially not surprising that the older clusters in the sample have more uncertain spectroscopic age measurements. In particular, as a cluster ages, the strength of the Balmer lines is significantly decreased (González Delgado et al. 2005). Finally, the most discrepant age estimates come from NGC 2623. This could be due to the fact that the galaxy has a complicated morphology (Evans et al. 2008). All of the young clusters identified come from a single "pie-wedge" structure to the right of the nucleus (see the Appendix), while all of the older clusters come from the nuclear regions. This makes using a simple prescription for an $A_{V}$ correction over the entire FOV more uncertain.

\subsubsection{Comparisons with Paschen- $\beta$ Equivalent Widths Derived from WFC3 Imaging}

K. L. Larson et al. (2017, in preparation) obtained Paschen- $\alpha$ and Paschen- $\beta(\mathrm{Pa} \beta)$ imaging for a subset of the GOALS sample, with six LIRGs (nine galaxies) overlapping our present $H S T$ sample. For any $B$-band cluster centroid that is spatially coincident with a high density clump in the $\mathrm{Pa} \beta$ images, we can directly compare our cluster ages to ages derived via the equivalent width (in angstroms) of the $\mathrm{Pa} \beta$ emission line. For an instantaneous burst SSP and a Salpeter IMF, the presence of $\mathrm{Pa} \beta$ emission constrains the burst age to less than $20 \mathrm{Myr}$ because stars with masses greater than $10 M_{\odot}$ are required for significant production of ionizing photons. We utilize Starburst99 models of $\mathrm{Pa} \beta$ equivalent width as a function of clump age to independently derive ages for 27 clusters in the sample (Leitherer et al. 1999).
From Figure 2, we find that approximately 78\% (21 of 27) of the clusters have ages that agree to within $\pm 0.3 \mathrm{dex}$, and $96 \%$ (26 of 27) have ages that agree to within \pm 0.6 dex. This shows us that the majority of all clusters we identify as having bright $\mathrm{Pa} \beta$ counterparts are indeed young. Additionally, $89 \%$ of the clusters ( $=24$ out of 27 ) which are photometrically identified as having ages less than $20 \mathrm{Myr}$ have a mean $\mathrm{Pa} \beta$ equivalent width of $\log (W(\mathrm{~Pa} \beta)[\AA]) \sim 1.7$ or $\log \left(\operatorname{Age}_{S B 99}(\right.$ years $\left.)\right) \sim 6.8$. It is important to note that of the 142 young $\left(t \leqslant 10^{7}\right.$ years) star clusters photometrically identified in these six LIRGs, we only associated a strong $\mathrm{Pa} \beta$ clump in the continuum-subtracted image with $19 \%$ (27 of 142) of them. This fraction is likely low for two reasons. (1) Our clusters are located primarily in the central regions of the galaxies, where the continuum subtraction is much more uncertain due to the larger contribution of diffuse large-scale NIR emission. As a result, the minimum equivalent width of a marginal $3 \sigma \mathrm{Pa} \beta$ detection can vary by a factor of a few within a galaxy and by almost an order of magnitude on a galaxy-by-galaxy basis. This variation corresponds to a $\sim 0.3 \mathrm{dex}$ change in the maximum derivable age using the SB99 model, which if we assume a 1:1 correlation, changes the age of the oldest cluster for which we would expect a counterpart in FUV emission by the same amount. (2) The resolution of the NIR $\mathrm{Pa} \beta$ images is 0 ". 12 /pixel, which is a factor of two lower than what we achieve in the FUV and optical imaging. This makes detecting bright compact sources of $\mathrm{Pa} \beta$ line emission embedded in a larger diffuse GMC cloud difficult at the distance of the galaxies in our sample.

Ultimately, both the local background subtraction and resolution contribute to the lack of overlap we observe in the $\mathrm{Pa} \beta$ and FUV emission. Regardless, this is an independent verification of our ability to derive accurate young ages for clusters in the sample, and shows us that our $A_{V}$ corrections can do a reasonable job at photometrically separating young and old clusters.

\subsection{Mass-Age Diagram and Completeness}

Figure 3 shows the derived age and corresponding mass of each cluster identified in the sample. An immediate observation one can make is the lack of low-mass, old clusters. This is due to the fact that clusters dim as they age and eventually become fainter than our UV detection limits. We also note the large number of clusters seen with ages below $10 \mathrm{Myr}$ over the full range of masses.

Although the cluster-fitting method can create some observed structure in the mass-age diagram, it is unlikely to do so over all masses at young ages. In particular, the lack of clusters with ages of $\sim 10^{7} \mathrm{Myr}$ is a common feature of modelderived mass-age diagrams of star clusters in galaxies (Gieles et al. 2005; Goddard et al. 2010). This is due to the limited age resolution and overall degeneracy of the $\mathrm{UV}-B, B-I$ color track at these ages (see the color-color diagrams in the Appendix). From the histograms in Figure 3, we conclude that there is a genuine overdensity of clusters with ages below $10 \mathrm{Myr}$ compared to above $10 \mathrm{Myr}$.

In order to determine the completeness limit of the cluster sample, we used a similar prescription to Whitmore et al. (1999), and set the limit for each galaxy as the magnitude at which $50 \%$ of the clusters are detected at $B$ and $I$, but are missed at FUV. The magnitude distributions for each band are corrected for foreground galactic extinction and spatially matched to the FOV of the SBC. Of the 22 LIRGs in the 
sample, 19 have magnitude distributions that span the full range of observed cluster values $\left(M_{B}=-10 \sim-15 \mathrm{mag}\right)$ and have a mean completeness of $M_{B} \sim-11.2 \mathrm{mag}$. The three remaining sources have completeness limits that are shifted to higher magnitudes $M_{B} \sim-13 \mathrm{mag}$, likely due to the fact that they are all farther away than the mean distance of the galaxies in the sample $(115 \mathrm{Mpc})$. It is important to note however, that there are several other galaxies for which a larger distance did not result in a shifted magnitude distribution, meaning that the actual $50 \%$ limit for the sample is not a strong function of the mean distance to any galaxy. Additionally, these outliers represent only $7 \%$ of the total cluster population. Therefore, to minimize their contributions to the final adopted limit for the entire sample, we calculated a cluster-weighted mean completeness limit and found that the mean shifted only slightly to $M_{B}=-11.26$.

By applying this completeness limit to the $\mathrm{BC} 03$ model, we can define regions of this parameter space (both as a function of cluster ages over a mass range and masses over an age range) where we are observationally complete and thus working with a mass-limited sample of clusters. Mass-limited cluster samples have the advantage over luminosity-limited samples because they recover the underlying shape of the age distribution, and are thus not affected by the distance to each galaxy. However, the total number of clusters can be highly uncertain simply because the lower-mass clusters are not included. We will discuss the implications of this fact in Section 5.

The four cuts were selected to sample distinct regions of the mass and age distribution for which we could maintain completeness. We define Region 1 to be

$$
\begin{gathered}
6<\log \left(M / M_{\odot}\right)<8, \\
6.5<\log (\tau)<8.7,
\end{gathered}
$$

Region 2 to be

$$
\begin{gathered}
5.3<\log \left(M / M_{\odot}\right)<6, \\
6.6<\log (\tau)<8,
\end{gathered}
$$

Region 3 to be

$$
\begin{gathered}
\log (\tau)<7, \\
5.3<\log \left(M / M_{\odot}\right)<8,
\end{gathered}
$$

and Region 4 to be

$$
\begin{gathered}
7.5<\log (\tau)<8.7, \\
6<\log \left(M / M_{\odot}\right)<8 .
\end{gathered}
$$

The two mass cuts are marked Regions 1 and 2 in the left panel of Figure 3. Since older clusters are intrinsically fainter, a higher mass limit will result in a cluster population that is mass limited to a wider range of ages. Note that the chosen mass regimes do not contain the youngest, least massive clusters that are only observed in a subset of our galaxies, and thus would bias any estimate for the global mass and age distributions of all the galaxies combined. Region 2 is chosen to match the age and mass limits from Fall et al. (2005), allowing us to make accurate comparisons to the cluster population of the most well-studied nearby major merger, the Antennae Galaxy. Regions 3 and 4 are chosen to sample the young ( $\leqslant 10 \mathrm{Myr}$ ) and old $\left(\tau \geqslant 10^{7.5}\right)$ clusters, respectively, within the completeness limit. When analyzing Regions 1,3 , and 4, we will exclude the largest mass bin of $\log \left(M / M_{\odot}\right)=8.0$. These very high masses are most likely the result of either an imperfect extinction correction or multiple star clusters in close proximity appearing as a single star cluster at the resolution of these images, resulting in a large derived total mass (See Section 5.2). Although clusters of these masses have rarely been observed in abundance, we note that Bastian et al. (2013) studied several young star clusters in NGC 7252 with masses greater than $10^{7} M_{\odot}$, including one cluster with a total mass of $\sim 10^{8} M_{\odot}$.

\section{Discussion}

After determining the ages, masses, and extinctions for the entire cluster sample, we directly compare these distributions with those of nearby normal and interacting galaxies. We focus on the interpretation of the derived cluster age distribution and mass function, and briefly discuss the implications for cluster formation efficiency. Ultimately, we discuss to what degree the differences observed in our cluster population can be attributed to the extreme star-forming environment unique to LIRGs in the local universe. Individual cluster age and mass functions for the most "cluster-rich" (i.e., greater than 25 detected clusters) galaxies are computed in Table 2.

\subsection{Age Distribution}

We consider the age distribution of clusters in our complete LIRG sample over the two mass ranges (i.e., Regions 1 and 2) described in Section 4.3. Specifically, we are interested in measuring the power-law index $\gamma$, where $d N / d \tau=\tau^{\gamma}$. Figure 4 is a plot of the logarithm of the number of clusters per time interval, $\log (d N / d \tau)$, versus the logarithm of the cluster age, $\log (\tau)$. The plotted data are binned by 0.4 in $\log (\tau)$ so as to fully encapsulate the model errors of 0.3 in $\log (\tau)$ discussed in Section 4.1. We see that a large fraction $(\sim 30 \%)$ of the clusters have ages less than 7.5 Myr. For the youngest, most massive clusters in the sample (contained in Region 1), a weighted linear least-squares fit to the cluster age distribution gives a power-law index of $\gamma=-0.9 \pm 0.3$, consistent with the derived power-law index for the Antennae Galaxies within $1 \sigma$ (Fall et al. 2005; Figure 2).

The distribution of the lower-mass clusters (Region 2) can be fit with a power-law index of $\gamma=-0.87 \pm 0.1$, also consistent with the derived power-law index for the Antennae Galaxies within $1 \sigma$. The change in $\gamma(\sim 0.04)$ for the solar and subsolar models was less than the uncertainty in the fit to the data in Figure 4. The similarity in the slope of the power-law index between the two mass cuts is also further confirmation that we are working in a mass-limited regime, where the slope of the age distribution does not get systematically flatter with increasing cluster mass or distance to the host galaxy (Bastian 2016).

Also plotted in Figure 4 are the age distributions for M83 and the LMC, normalized to the fitted number of clusters in the youngest age bin. As can be seen, $\gamma$ for the LIRG sample is steeper than what is measured for these lower-mass, normal star-forming systems. In addition, Adamo \& Bastian (2015) provide a table summary of $\gamma$ for several local galaxies; in all cases, $\gamma$ is flatter than -1 .

There are two possible interpretations of this plot:

(1) If a continuous (or near-continuous) cluster formation rate is assumed during the merging process for each LIRG, then the index of $\gamma=-1$ is an indication that $90 \%$ of the clusters 
formed are disappearing every age dex. In the case of the Antennae Galaxies, Fall et al. (2005) concluded that the majority of the clusters are rapidly disrupted within the merger via "infant mortality." This scenario not only seems to fit into the nature of the violent environments of galaxy mergers, but may also explain the negative value of $\gamma$ (albeit not as negative as measured for mergers) observed in lower-mass, less starforming, quiescent nearby spirals.

We note that when discussing "infant mortality," it is important to mention that the rapid decrease in the number of clusters as a function of age could be due to the inclusion of young, low-density, unbound OB associations in cluster catalogs (e.g., Bastian et al. 2012; Silva-Villa et al. 2014). When these associations are removed, the age distributions for local star-forming galaxies appear to flatten. Kruijssen \& Bastian (2016) point out that these effects can be minimized by selecting slightly older clusters $(10-50 \mathrm{Myr})$, so that associations will have already been dispersed into the field. If this were a dominant effect in our sample, we would expect the age distribution of Region 2 to be much flatter and inconsistent with the Antennae value. Further, although we cannot verify the amount of contamination from $\mathrm{OB}$ associations for the youngest clusters $(t<10 \mathrm{Myr})$ in our sample, the high mass cutoff for Region 1 ensures that this effect is minimized.

(2) The star formation rate has increased such that the bulk of the star formation, and cluster formation, has happened fairly recently as a result of the interaction of the two galaxies. This seems unlikely due to the fact that many of the galaxies within the sample have been interacting for a few hundred million years, whereas the median age of clusters for the whole sample is only $\sim 10^{7}$ years. Hopkins et al. (2013) finds that when simulations use realistic prescriptions for galaxy feedback, the star formation in a galaxy merger can in fact be time variable and drops between each passage. Therefore, the average SF enhancement is only ever a factor of a few during the course of a merger, which is not enough to explain the $90 \%$ decrease in the number of clusters at each age dex (Karl et al. 2011). We could assume that all of the galaxies across the various merger stages are being viewed at these bursty peaks in the star formation rate, but we also consider this an unlikely scenario.

Under this framework, we would also be forced to accept that the star formation rates in nearby normal galaxies (which have negative $\gamma$ values - though note the above discussion of possible OB association contamination) are also increasing. In well-studied star-forming galaxies like the Milky Way and the Magellanic Clouds, the SFR is observed to have been nearly constant over the last Gyr, which argues strongly for the fact that the decline in $d N / d \tau$ is primarily a consequence of disruption in the Milky Way and Magellanic Clouds (Harris \& Zaritsky 2009; Chandar et al. 2010).

Given the above, the most plausible explanation is that clusters are being rapidly destroyed in luminous galaxy mergers at a rate that exceeds the cluster destruction process occurring in nearby normal galaxies.

\subsection{Mass Function}

The cluster mass function (CMF) has the form $d N / d M \sim M^{\beta}$. For star clusters in our sample, this was derived by stacking the mass distributions of each galaxy, keeping the binning constant $(0.4$ in $\log (M))$, and then performing a cluster-weighted linear least-squares fit as a function of derived mass. For clusters with ages $t \leqslant 10^{7}$ years and $t \sim 10^{8}$ years, we derive a mass function with $\beta=-1.95 \pm 0.11$ and $-1.67 \pm 0.33$, respectively (see Figure 5). In comparison, $\beta$ is commonly measured to be -2 for the majority of lower luminosity star-forming galaxies, as well as the Antennae Galaxies (Larsen 2010). The change in $\beta$ for the solar and subsolar models was less than the uncertainty in the fit (i.e., $<0.1)$ to the data in Figure 5.

An alternative approach to modeling the ICMF is with a twocomponent Schechter function of the form $d N / d M=$ $\left(M / M_{c}\right)^{\alpha} e^{\left(M / M_{c}\right)}$. For reference, the $M_{c}$, or characteristic mass, measured for the Milky Way is $\sim 10^{5} M_{\odot}$ (Bastian 2008b). If we assumed that a star formation rate of $\sim 100 M_{\odot} \mathrm{yr}^{-1}$ went into forming only clusters, the number of clusters with $M \geqslant 10^{7} M_{\odot}$ would still be negligible for $M_{c}=10^{5} M_{\odot}$, even if these high SFRs could be sustained for $\sim 100$ Myr. Thus, the mere presence of $10^{7} M_{\odot}$ clusters in our sample indicates that the cluster formation environment in more extreme systems is different than that observed in lower luminosity spiral galaxies.

Larsen (2010) shows that a Schechter function with a canonical -2 power-law slope and $M_{c}=10^{6.3} M_{\odot}$ can reproduce the observed distribution in the Antennae Galaxies equally well. In Figure 5, it is clear that we cannot simply adopt these parameters to fit our observations. Instead, we require both a slightly shallower power-law slope and a slightly larger cutoff mass due primarily to the fact that we are observing clusters with masses greater than $10^{6.5} M_{\odot}$, which simply are not observed in the Antennae. It is important to note that our data (Region $3+4$ ) is consistent to within $1 \sigma$ of a -2 power law in $d N / d M$ over the same mass range as the Antennae, but can also be fit at the highmass end using a modified Schechter function with a cutoff mass of $10^{7} M_{\odot}$. This is clearly larger than what has been recently observed in $\mathrm{M} 31$, where the observed cutoff mass for the cluster sample is $M_{c} \sim 8 \times 10^{3} M_{\odot}$ (Johnson et al. 2017). Interestingly, in that work, the authors define a relationship for the expected $M_{c}$ as a function of $\Sigma_{\mathrm{SFR}}$ as $\log M_{c}=(1.07 \pm 0.10) \times \log \Sigma_{\mathrm{SFR}}+(6.82 \pm 0.20)$. If the typical value of $\Sigma_{\mathrm{SFR}}$ for the LIRGs in the GOALS sample is used ( $\mathrm{U}$ et al. 2013), we expect $M_{c} \sim 10^{7} M_{\odot}$, which is consistent with our derived fit and indicates that high-mass clusters can indeed form more efficiently in higher starforming environments.

When interpreting these results, it is important to consider several possible factors that could affect our derived mass functions:

(1) If lower-mass star clusters are preferentially disrupted, the mass distribution of the surviving star clusters in a merger remnant will be shallower than what is observed in a quiescent spiral galaxy (Kruijssen et al. 2012; Li et al. 2017). We might also expect this to correspond to a steeper age distribution for the lower-mass cluster sample (Figure 4; Region 2), but given that our "low-mass" clusters are still rather massive, the lack of a clear difference in $d N / d \tau$ is not surprising. Therefore, the cluster disruption in these galaxies appears to be mostly mass independent (i.e., we find that $\gamma \sim-1$ over the mass range of $M_{\odot}=10^{5}-10^{6}$ ), a finding that Whitmore et al. (2010) confirmed for the Antennae over the same range of cluster masses (Figure 5; yellow track).

When we increase the lower limit cluster mass for Region 1 to $10^{6.5} M_{\odot}$ in Figure 4, we observe a disruption rate of $d N / d \tau \sim \tau^{-0.75 \pm 0.4}$. This leads us to conclude that cluster disruption in LIRGs appears largely consistent with what is seen in the Antennae up to $10^{6.5} M_{\odot}$. We note that the 
uncertainty on the measured slope is much larger than for Region 2, so in principle, gamma could be shallower than the Antennae Galaxies in this mass regime. However, if this were a strong effect in our data, we would expect our observed CMF in Region 3 to be shallower than the -2 power law used to represent the underlying ICMF.

(2) The choice of bin size for our data could systematically flatten the measured $\beta$ (Maíz Apellániz \& Úbeda 2005). We use bin sizes in mass and age of $0.4 \operatorname{dex}$ in $\log (M)$ and $\log (t)$, chosen to fully encapsulate the typical uncertainty associated with our age and subsequent mass estimations. To test the effect this choice has on the measured slope, we explored two other bin sizes, 0.2 and $0.6 \mathrm{dex}$. We found that the slopes derived for $d N / d \tau$ and $d N / d M$ change on average by $0.1-0.2$ dex. As this is comparable with the $1 \sigma$ uncertainties on each slope measurement, we conclude that our choice of bin size is not significantly affecting our determination of the shape of the cluster mass distribution.

(3) At the resolution of our observations, multiple lowermass clusters may appear as one massive cluster, and thus systematically flatten the CMF. To test this possibility, we ran Source Extractor on $B$-band and $I$-band WFC images of NGC 4038/9 from the Hubble Legacy Archive (HLA) to identify star clusters. The distance used for NGC $4038 / 9$ is $\sim 24 \mathrm{Mpc}$, but the median distance of our sample is four times farther away. Since the pixel scale of the Drizzlepac output images is the same, we simply smoothed the HLA images with a boxcar function of 4 pixels. Source Extractor was then run on this smoothed image with the Source Extractor results from the original, pre-smoothed images as a reference. For this step, Source Extractor only outputs sources that are both identified in the smooth image and also match a source in the original list (within a search radius of 4 pixels, i.e., the same size as the smoothing). The ratio $\left(N_{\text {orig }}-N_{\text {smoothed }}\right) / N_{\text {orig }}$ should give an upper limit for the fraction of dual sources identified as 1 in the smoothed image.

For the $B$-band and $I$-band image comparisons, this ratio is 0.3 and 0.26 , respectively. Thus, roughly $30 \%$ of the "blended clusters" identified in our LIRGs with $D \geqslant 100 \mathrm{Mpc}$ would actually be identified as a complex of single clusters at the resolution of the Antennae. By redistributing to the lower-mass end this percentage of clusters, with masses greater than $5 \times 10^{6} M_{\odot}$, we observe a steepening of the mass function of $\sim 0.1$ dex. Despite this fact, it is clear that the existence of young high-mass $\left.\geqslant 10^{7} M_{\odot}\right)$ clusters in our sample cannot be solely attributed to a resolution limit. Finally, it is worth noting that cluster blending can affect the estimated cluster ages. The effect most likely pushes clusters toward the median cluster age, and thus if deblending randomly populates the young and old cluster parts of the age distribution, there will not be a dramatic effect on $\gamma$.

Given the above, it appears that the differences in the slope observed in the LIRG sample relative to the Antennae mass function are not caused by mass-dependent cluster disruption from $10^{5}-10^{6.5} M_{\odot}$. When we consider the effect of a resolution limit on the high-mass end of the distribution, we can reconcile the small discrepancies in the observed slopes. Therefore, cluster formation in these galaxies can be explained with a universal -2 power-law fit to the mass distribution up to at least $10^{6.5} M_{\odot}$. However, we emphasize that the prevalence of the most massive clusters observed in the sample is compelling evidence that these clusters exist more predominantly in the more extreme star-forming environments of LIRGs.

This idea is further supported by the fact that a Schechter function, with $M_{c} \sim 10^{7} M_{\odot}$, can also fit our data over the full range of observed cluster masses relative to a simple power-law formalism. This implies that GMCs in LIRGs can have higher ISM pressures and densities than what has been seen in other galactic environments. Recently, Maji et al. (2016) used hydrodynamic simulations of two equal-mass Milky Way-like merging galaxies to show that such ISM conditions are actually capable of producing clusters in the range of $10^{5.5-7.5} M_{\odot}$ (Figure 4), consistent with the mass scales we observe in our LIRG sample.

\subsection{Merger Stage Dependence}

Since our LIRG sample spans the full range of merger stages, we can test if our explanation of cluster formation and destruction depends on the dynamical state of the galaxy. Haan et al. (2013), Kim et al. (2013), and Stierwalt et al. (2013) have classified the merger stage of each U/LIRG in the GOALS sample based on their morphological appearance at multiple wavelengths. These merger classification schemes run from pre-first passage to single coalesced nuclei. We separated the sample into early (classes 0-2), middle (classes 3-4), and latestage (classes 5-6) mergers. In order to quantify any differences in each age distribution, we ran a KS-test comparing the normalized distributions of the early, middle, and late-stage mergers to the total sample. We find that within our subsample of GOALS LIRGs, these individual merger stage distributions are drawn from the same parent distribution of ages with a $92 \%$ probability or higher.

For galaxies classified across all merger stage bins, we find that the most massive clusters in the sample (Region 1) are always consistent with a -1 power law in $d N / d \tau$, which is further justification for combining the cluster populations for each galaxy into a single sample, and indicates that disruption does not vary much, within the uncertainty, throughout the merger. It also provides credence to the idea that the SFR of a merging galaxy is bursty, which, given the large size of our age bins, is an effect on the age distribution we can safely ignore. This allows us to characterize each galaxy as having an elevated but roughly continuous SFR.

When breaking the sample down to early- and mid-stage mergers in Figure 6, we find that star clusters in both early- and mid-stage mergers show a power-law distribution of $d N / d M \sim$ $M^{-1.8}$ across both age regimes. Additionally, each mass function is normalized by the total duration within their respective age bins in order to remove any artifacts of the bins having different time ranges. This helps to emphasize that the number of clusters that survive decreases in absolute number and independent of mass from the pre- to the ongoing-merger systems.

Under the assumption of a constant SFR, the youngest clusters in each galaxy merger class will show the same slope in $d N / d M$. Our results are consistent with the idea that the star formation history is not changing significantly between merger stages, and thus cannot be a dominant effect in driving the observed age distributions we see for our sample, when combing all galaxies together.

Additionally, when analyzing the cluster mass distribution, we assumed that the formation conditions (i.e., characteristic mass $M_{c}$ and slope $\alpha$ ) do not change significantly throughout 
the merging process. The similarity of the slopes between each merger class is consistent with simulations of merging disk galaxies, which find that the characteristic mass $M_{c}$ evolves at a rate of only $\sim 0.3-0.4$ dex $\mathrm{Gyr}^{-1}$ (Kruijssen et al. 2012).

\subsection{Cluster Formation Efficiency}

Finally, we consider the efficiency of cluster formation (CFE) within the high star formation rate environments of LIRGs. CFE, or $\Gamma$, is defined the ratio of the rate of stellar mass formation in bound clusters, $\dot{M}_{\mathrm{SC}}$, to the global star formation rate, $\dot{M}_{\mathrm{SF}}$, over the same time interval, i.e.,

$$
\Gamma=\frac{\dot{M}_{\mathrm{SC}}}{\dot{M}_{\mathrm{SF}}} \times 100 \% .
$$

For our sample, the fact that we do not detect clusters well below $10^{5} M_{\odot}$, and that we have significant cluster disruption over all masses, makes the estimation of the cluster formation efficiency (CFE) highly uncertain.

This is compounded by the fact that our UV-bright cluster population is not sampling the full SFR as traced by the total UV+IR-based SFR measurements from Howell et al. (2010). Additionally, we cannot match our UV-based CFR to the total GALEX UV SFR estimation because the field of view of the $\mathrm{SBC}$ is $\sim 1 / 140$ that of $G A L E X$, and thus a correction for the clusters we miss is uncertain. The large amount of obscuring dust also makes a completeness correction to derive a total mass and CFR based on our mass distributions difficult for our LIRG sample. Johnson et al. (2016) notes that CFE calculations are best done in dust-free environments that show little sign of significant cluster disruption, a scenario we are simply not presented with in our sample. Therefore, we leave a discussion about CFE in LIRGs to future studies involving deep IR-based observations that have both a larger FOV and the ability to detect more dustenshrouded low-mass clusters.

\section{Summary}

Hubble Space Telescope ACS/HRC FUV (F140LP) and ACS/WFC optical (F435W and F814W) observations of a sample of 22 star cluster-rich LIRGs in the GOALS sample were obtained. These observations have been utilized to derive the ages and masses of the star clusters contained within these systems in order to examine the cluster properties in extreme starburst environments relative to those in nearby, lower luminosity star-forming galaxies. The following conclusions are reached.

(1) We have detected 665 clusters within the inner $30^{\prime \prime} \times 30^{\prime \prime}$ of these 22 LIRGs (27 nuclei). These clusters have $S / N \geqslant 5$ in all three filters and deconvolved FWHMs of $\leqslant 2$ pixels as measured by ISHAPE.

(2) Cluster ages have been derived by assuming an instantaneous SSP, Salpeter IMF, and either a solar or subsolar metallicity. By requiring the derived cluster ages to be consistent when using both a color-color and SED-based fitting technique, we obtain a final sample of 484 clusters whose properties are reliably constrained within the $1 \sigma$ uncertainties of the SSP models. The derived cluster ages imply a disruption rate of $d N / d \tau=\tau^{-0.9+/-0.3}$ for cluster masses $\geqslant 10^{6} M_{\odot}$ and $d N / d \tau=\tau^{-0.87+/-0.08}$ for cluster masses $10^{5.3}<M<10^{6} M_{\odot}$. This is consistent with what is seen in the Antennae, and indicates the general influence mergers have on the creation and destruction of star clusters. The measured $\gamma$ is steeper than that measured for lower-mass, less star-forming systems in the local universe, implying that the merging process produces a fundamentally different cluster disruption law.

(3) We have identified a large number of $M \geqslant 10^{6} M_{\odot}$ clusters in the sample, which indicates that the more extreme star-forming environments of LIRGs are capable of producing more high-mass clusters than what is observed in galaxies like the Milky Way or even the Antennae (Larsen 2009; Whitmore et al. 2010; Bastian et al. 2012). The derived cluster masses also imply a CMF for the sample of $d N / d M=M^{-1.95+/-0.11}$, which is consistent with a -2 power law in $d N / d M$. Together with the fact that we do not see a significant change in the age distribution slope as a function of mass, we interpret our mass function slope as evidence against massdependent cluster disruption at $M \geqslant 10^{5.3} M_{\odot}$, which would flatten the observed $\mathrm{CMF}$ relative to a canonical -2 power law in this regime.

The authors thank B. Whitmore, R. Chandar, A. Mulia, and G. Soutchkova for useful discussions and assistance. The authors also thank the referee for detailed comments and suggestions, which have improved the manuscript. S.T.L. was supported by the NASA VSGC Graduate Fellowship. A.S.E., D.C.K., S. M., A. A., A. B., A. Y., and T. L. were supported by NSF grant AST 1109475 and by NASA through grants HSTGO10592.01-A, HST-GO11196.01-A, and HST-GO13364 from the Space Telescope Science Institute, which is operated by the Association of Universities for Research in Astronomy, Inc., under NASA contract NAS5-26555. G.C.P. was supported by a FONDECYT Postdoctoral Fellowship (No. 3150361). A.S.E. was also supported by the Taiwan, R.O.C. Ministry of Science and Technology grant MoST 102-2119-M001-MY3. T.D.-S. acknowledges support from ALMA-CONICYT project 31130005 and FONDECYT regular project 1151239.

Portions of this work were performed at the Aspen Center for Physics, which is supported by National Science Foundation grant PHY-1066293. This work was partially supported by a grant from the Simons Foundation. Finally, This research has made use of the NASA/IPAC Extragalactic Database (NED), which is operated by the Jet Propulsion Laboratory, California Institute of Technology, as well as the National Radio Astronomy Observatory, which is a facility of the National Science Foundation operated under cooperative agreement by Associated Universities Inc.

\section{Appendix Galaxy Descriptions}

In the following sections, we give a brief description of the basic morphology and star cluster spatial distributions within each galaxy, as well as the adopted values for the maximum amount of visual extinction we use in our model. See A. S. Evans et al. (2017, in preparation) for a detailed description of all 88 LIRGs in the GOALS sample that have been observed with $H S T$. 
Table 3

Observed Properties of Star Clusters in NGC 0017

\begin{tabular}{|c|c|c|c|c|c|c|c|c|}
\hline ID & R.A. & Decl. & $M_{B}$ & $\sigma_{B}$ & $M_{I}$ & $\sigma_{I}$ & $M_{\mathrm{FUV}}$ & $\sigma_{\mathrm{FUV}}$ \\
\hline 1 & 2.776819641 & -12.10616498 & -13.81 & 0.01 & -14.87 & 0.01 & -12.67 & 0.03 \\
\hline 2 & 2.777320196 & -12.10713204 & -13.89 & 0.01 & -14.53 & 0.01 & -14.20 & 0.01 \\
\hline 3 & 2.777273773 & -12.10658526 & -11.05 & 0.06 & -11.91 & 0.05 & -10.84 & 0.18 \\
\hline 4 & 2.777432692 & -12.10695518 & -13.56 & 0.01 & -14.43 & 0.01 & -12.32 & 0.05 \\
\hline 5 & 2.777660645 & -12.10636335 & -12.56 & 0.01 & -13.44 & 0.01 & -12.68 & 0.03 \\
\hline 6 & 2.778745035 & -12.10880075 & -14.29 & 0.01 & -15.27 & 0.01 & -12.96 & 0.02 \\
\hline 7 & 2.777920896 & -12.10509066 & -12.03 & 0.01 & -12.76 & 0.01 & -11.67 & 0.08 \\
\hline 8 & 2.778717571 & -12.10546794 & -10.72 & 0.02 & -11.38 & 0.02 & -11.27 & 0.12 \\
\hline 9 & 2.779140091 & -12.10608216 & -15.05 & 0.01 & -15.86 & 0.01 & -15.36 & 0.01 \\
\hline 10 & 2.779887259 & -12.10772035 & -11.65 & 0.01 & -12.39 & 0.01 & -11.62 & 0.16 \\
\hline 11 & 2.780845501 & -12.10807908 & -10.35 & 0.03 & -11.11 & 0.03 & -11.06 & 0.02 \\
\hline 12 & 2.777023706 & -12.10749699 & -13.10 & 0.04 & -14.80 & 0.16 & -11.10 & 0.14 \\
\hline 13 & 2.776766427 & -12.10731248 & -13.23 & 0.02 & -14.20 & 0.02 & -13.08 & 0.02 \\
\hline 14 & 2.776532232 & -12.10983287 & -11.54 & 0.01 & -12.36 & 0.01 & -11.13 & 0.08 \\
\hline 15 & 2.776287946 & -12.10888848 & -13.35 & 0.01 & -14.04 & 0.01 & -13.32 & 0.02 \\
\hline
\end{tabular}

Table 4

Derived Properties of Star Clusters in NGC 0017

\begin{tabular}{|c|c|c|c|c|c|c|}
\hline ID & $\log ($ Age $)$ & $\sigma_{\text {Age }}$ & $\log \left(M / M_{\odot}\right)$ & $\sigma_{M}$ & $A_{V}$ & $\sigma_{A_{V}}$ \\
\hline 1 & 8.61 & 0.02 & 7.34 & 0.16 & 0.20 & $\overline{0.05}$ \\
\hline 2 & 8.36 & 0.02 & 7.11 & 0.17 & 0.10 & 0.06 \\
\hline 3 & 8.46 & 0.58 & 6.04 & 0.61 & 0.10 & 0.63 \\
\hline 4 & 8.66 & 0.02 & 7.18 & 0.16 & 0.01 & 0.04 \\
\hline 5 & 6.66 & 0.81 & 6.18 & 0.70 & 1.90 & 0.77 \\
\hline 6 & 8.66 & 0.69 & 7.47 & 0.16 & 0.01 & 5.27 \\
\hline 7 & 6.66 & 0.03 & 6.08 & 0.16 & 2.10 & 0.04 \\
\hline 8 & 6.66 & 0.79 & 5.29 & 0.69 & 1.60 & 0.74 \\
\hline 9 & 6.64 & 0.10 & 7.10 & 0.16 & 1.80 & 5.62 \\
\hline 10 & 6.66 & 2.37 & 5.87 & 0.16 & 2.00 & 4.73 \\
\hline 11 & 6.34 & 0.86 & 5.65 & 0.67 & 2.10 & 0.71 \\
\hline 12 & 8.46 & 0.18 & 7.55 & 0.52 & 1.40 & 0.51 \\
\hline 13 & 8.36 & 0.29 & 7.00 & 0.37 & 0.40 & 0.33 \\
\hline 14 & 8.51 & 1.93 & 6.23 & 0.16 & 0.01 & 0.03 \\
\hline 15 & 8.46 & 5.32 & 6.91 & 0.16 & 0.01 & 0.78 \\
\hline
\end{tabular}

Table 5

Observed Properties of Star Clusters in Arp 256S

\begin{tabular}{|c|c|c|c|c|c|c|c|c|}
\hline ID & R.A. & Decl. & $M_{B}$ & $\sigma_{B}$ & $M_{I}$ & $\sigma_{I}$ & $M_{\mathrm{FUV}}$ & $\sigma_{\mathrm{FUV}}$ \\
\hline 1 & 4.713679548 & -10.37685826 & -10.41 & 0.05 & -11.03 & 0.07 & -12.97 & 0.05 \\
\hline 2 & 4.710809481 & -10.37888736 & -12.54 & 0.01 & -12.61 & 0.02 & -14.38 & 0.01 \\
\hline 3 & 4.710599656 & -10.377146 & -10.45 & 0.13 & -10.34 & 0.22 & -13.10 & 0.04 \\
\hline 4 & 4.711341331 & -10.37839176 & -11.99 & 0.02 & -12.79 & 0.02 & -13.31 & 0.03 \\
\hline 5 & 4.71074686 & -10.37664793 & -12.51 & 0.03 & -13.46 & 0.04 & -14.17 & 0.03 \\
\hline 6 & 4.711535325 & -10.37750998 & -13.53 & 0.02 & -13.80 & 0.02 & -15.23 & 0.02 \\
\hline 7 & 4.712724227 & -10.37952183 & -10.43 & 0.15 & -11.84 & 0.08 & -12.65 & 0.13 \\
\hline 8 & 4.712065091 & -10.37821862 & -12.93 & 0.03 & -13.21 & 0.04 & -14.27 & 0.03 \\
\hline 9 & 4.712819126 & -10.37928414 & -11.79 & 0.05 & -12.05 & 0.09 & -13.16 & 0.08 \\
\hline 10 & 4.713033719 & -10.37882884 & -12.69 & 0.02 & -12.63 & 0.05 & -15.17 & 0.02 \\
\hline 11 & 4.712218761 & -10.37674074 & -14.31 & 0.02 & -14.61 & 0.02 & -15.75 & 0.01 \\
\hline 12 & 4.712398738 & -10.3769871 & -12.19 & 0.03 & -12.13 & 0.21 & -13.01 & 0.05 \\
\hline 13 & 4.712719547 & -10.37756498 & -11.80 & 0.06 & -11.67 & 0.12 & -12.94 & 0.10 \\
\hline 14 & 4.71300344 & -10.37787256 & -12.42 & 0.03 & -13.03 & 0.03 & -13.11 & 0.04 \\
\hline 15 & 4.71191981 & -10.37588942 & -12.99 & 0.03 & -12.56 & 0.06 & -15.11 & 0.01 \\
\hline 16 & 4.713366435 & -10.37755202 & -13.26 & 0.05 & -13.56 & 0.07 & -14.79 & 0.03 \\
\hline 17 & 4.713006219 & -10.37670878 & -11.65 & 0.10 & -11.37 & 0.15 & -13.00 & 0.05 \\
\hline 18 & 4.712859587 & -10.37631389 & -11.93 & 0.03 & -12.60 & 0.05 & -13.26 & 0.04 \\
\hline 19 & 4.713776161 & -10.37757731 & -12.27 & 0.04 & -12.18 & 0.04 & -13.85 & 0.06 \\
\hline 20 & 4.713381893 & -10.37643331 & -10.68 & 0.07 & -10.71 & 0.14 & -13.35 & 0.03 \\
\hline
\end{tabular}


Table 6

Derived Properties of Star Clusters in Arp 256S

\begin{tabular}{|c|c|c|c|c|c|c|}
\hline ID & Log(Age) & $\sigma_{\text {Age }}$ & $\log \left(M / M_{\odot}\right)$ & $\sigma_{M}$ & $A_{V}$ & $\sigma_{A_{V}}$ \\
\hline 1 & 6.88 & 0.17 & 4.60 & 0.20 & 0.10 & 0.07 \\
\hline 2 & 6.64 & 0.02 & 5.51 & 0.18 & 0.70 & 0.05 \\
\hline 3 & 5.10 & 0.43 & 5.10 & 0.33 & 0.80 & 0.23 \\
\hline 5 & 7.54 & 0.28 & 6.24 & 0.26 & 0.40 & 0.18 \\
\hline 6 & 6.46 & 0.27 & 6.49 & 0.33 & 1.30 & 0.27 \\
\hline 7 & 5.10 & 0.09 & 5.57 & 0.29 & 1.70 & 0.16 \\
\hline 10 & 6.40 & 0.64 & 5.78 & 0.35 & 0.80 & 0.30 \\
\hline 11 & 6.60 & 0.06 & 6.34 & 0.23 & 1.00 & 0.15 \\
\hline 12 & 6.66 & 0.25 & 5.66 & 0.28 & 1.20 & 0.21 \\
\hline 13 & 6.66 & 0.72 & 5.40 & 0.21 & 1.00 & 0.10 \\
\hline 14 & 6.66 & 0.81 & 5.92 & 0.68 & 1.50 & 0.73 \\
\hline 15 & 6.66 & 0.76 & 5.56 & 0.18 & 0.40 & 0.04 \\
\hline 20 & 6.74 & 0.38 & 4.45 & 0.31 & 0.01 & 0.24 \\
\hline
\end{tabular}

Table 7

Observed Properties of Star Clusters in Arp 256N

\begin{tabular}{|c|c|c|c|c|c|c|c|c|}
\hline ID & R.A. & Decl. & $M_{B}$ & $\sigma_{B}$ & $M_{I}$ & $\sigma_{I}$ & $M_{\mathrm{FUV}}$ & $\sigma_{\mathrm{FUV}}$ \\
\hline 1 & 4.705283874 & -10.36593186 & -10.52 & 0.03 & -10.40 & 0.07 & -12.96 & 0.03 \\
\hline 2 & 4.706681456 & -10.36309635 & -11.05 & 0.02 & -11.05 & 0.08 & -13.47 & 0.03 \\
\hline 3 & 4.706997794 & -10.36259164 & -12.33 & 0.02 & -12.88 & 0.02 & -14.58 & 0.04 \\
\hline 4 & 4.708060796 & -10.36212902 & -11.79 & 0.03 & -12.41 & 0.04 & -13.77 & 0.02 \\
\hline 5 & 4.707757416 & -10.3616235 & -11.16 & 0.06 & -10.74 & 0.22 & -12.72 & 0.06 \\
\hline 6 & 4.708684462 & -10.36263369 & -12.69 & 0.01 & -13.05 & 0.02 & -14.56 & 0.01 \\
\hline 7 & 4.708763384 & -10.36246337 & -11.99 & 0.02 & -12.38 & 0.03 & -14.05 & 0.03 \\
\hline 8 & 4.710151015 & -10.36378048 & -11.10 & 0.03 & -11.14 & 0.06 & -13.44 & 0.03 \\
\hline 9 & 4.708704027 & -10.36100592 & -12.08 & 0.05 & -12.17 & 0.03 & -14.37 & 0.04 \\
\hline 10 & 4.709365918 & -10.361838 & -12.00 & 0.04 & -12.08 & 0.13 & -14.18 & 0.03 \\
\hline 11 & 4.708851609 & -10.3608711 & -13.78 & 0.01 & -13.85 & 0.02 & -16.17 & 0.01 \\
\hline 12 & 4.710324609 & -10.36344198 & -11.80 & 0.02 & -12.23 & 0.02 & -13.13 & 0.06 \\
\hline 13 & 4.707903866 & -10.35832277 & -13.74 & 0.01 & -14.07 & 0.02 & -15.62 & 0.01 \\
\hline 14 & 4.708214025 & -10.3587839 & -11.98 & 0.02 & -12.23 & 0.04 & -13.66 & 0.02 \\
\hline 15 & 4.708155895 & -10.358066 & -13.40 & 0.01 & -13.49 & 0.02 & -15.67 & 0.01 \\
\hline 16 & 4.709517938 & -10.35699562 & -11.91 & 0.02 & -12.51 & 0.03 & -13.73 & 0.02 \\
\hline
\end{tabular}

\section{NGC 0017}

NGC 0017 is a late-stage merger that contains a single resolved nucleus surrounded by dust lanes associated with spiral arms in the inner few kiloparsecs. Several bright star clusters are visible within this nuclear spiral region (Figure 7). The maximum $A_{V}$ adopted for this galaxy is $3.0 \mathrm{mag}$ of visual extinction. (see Figure 8; Dametto et al. 2014). The observed and derived properties for the star clusters identified in this galaxy are given in Tables 3 and 4, respectively.

\section{Arp 2565}

Arp 256 is a mid-stage merger containing a southern (MCG02-01-051) and northern (MCG-02-01-052) galaxy. Arp 256S has an elongated $\sim 1^{\prime \prime}(400 \mathrm{pc})$ nucleus, and the north and southwest tails contain the majority of the star clusters in the galaxy (Figure 9). The maximum $A_{V}$ adopted for this galaxy is 1.7 mag of visual extinction (see Figure 10; Smith et al. 2014). The observed and derived properties for the star clusters identified in this galaxy are given in Tables 5 and 6, respectively.

\section{Arp 256N}

Arp $256 \mathrm{~N}$ has a central, point-like nucleus. The majority of the star clusters are seen along the tidal tails in this galaxy (Figure 11). The maximum $A_{V}$ adopted for this galaxy is 1.7 mag of visual extinction (see Figure 12; Smith et al. 2014). The observed and derived properties for the star clusters identified in this galaxy are given in Tables 7 and 8, respectively. 
Table 8

Derived Properties of Star Clusters in Arp 256N

\begin{tabular}{|c|c|c|c|c|c|c|}
\hline ID & Log(Age) & $\sigma_{\text {Age }}$ & $\log \left(M / M_{\odot}\right)$ & $\sigma_{M}$ & $A_{V}$ & $\sigma_{A_{V}}$ \\
\hline 1 & 6.54 & 0.08 & 4.77 & 0.22 & 0.70 & 0.13 \\
\hline 2 & 6.66 & 0.19 & 5.00 & 0.30 & 0.60 & 0.23 \\
\hline 3 & 7.42 & 0.21 & 5.84 & 0.21 & 0.10 & 0.11 \\
\hline 4 & 7.32 & 0.25 & 5.73 & 0.25 & 0.40 & 0.17 \\
\hline 5 & 6.52 & 0.01 & 5.29 & 0.21 & 1.20 & 0.10 \\
\hline 6 & 7.76 & 0.53 & 5.68 & 0.47 & 0.01 & 0.44 \\
\hline 7 & 7.63 & 0.45 & 5.34 & 0.36 & 0.01 & 0.31 \\
\hline 8 & 6.64 & 0.11 & 5.07 & 0.26 & 0.70 & 0.19 \\
\hline 9 & 6.66 & 0.27 & 5.47 & 0.29 & 0.70 & 0.22 \\
\hline 10 & 6.44 & 0.16 & 5.70 & 0.28 & 1.10 & 0.21 \\
\hline 11 & 6.68 & 0.26 & 6.09 & 0.24 & 0.60 & 0.15 \\
\hline 12 & 6.44 & 0.41 & 5.94 & 0.45 & 1.70 & 0.42 \\
\hline 13 & 6.72 & 0.49 & 6.16 & 0.42 & 0.80 & 0.39 \\
\hline 14 & 6.48 & 0.07 & 5.79 & 0.22 & 1.40 & 0.12 \\
\hline 15 & 5.70 & 0.44 & 6.52 & 0.29 & 1.10 & 0.23 \\
\hline 16 & 6.76 & 0.33 & 5.36 & 0.30 & 0.70 & 0.24 \\
\hline
\end{tabular}

Table 9

Observed Properties of Star Clusters in NGC 0695

\begin{tabular}{|c|c|c|c|c|c|c|c|c|}
\hline ID & R.A. & Decl. & $M_{B}$ & $\sigma_{B}$ & $M_{I}$ & $\sigma_{I}$ & $M_{\mathrm{FUV}}$ & $\sigma_{\mathrm{FUV}}$ \\
\hline 1 & 27.80620232 & 22.58288641 & -11.35 & 0.06 & -12.24 & 0.06 & -11.68 & 0.08 \\
\hline 2 & 27.8065178 & 22.57996324 & -11.21 & 0.02 & -11.65 & 0.03 & -11.93 & 0.06 \\
\hline 3 & 27.80625132 & 22.58196385 & -10.37 & 0.04 & -10.99 & 0.04 & -11.29 & 0.11 \\
\hline 4 & 27.80849949 & 22.58172092 & -10.96 & 0.10 & -11.58 & 0.10 & -11.85 & 0.06 \\
\hline 5 & 27.80866289 & 22.58234757 & -13.19 & 0.02 & -13.45 & 0.04 & -13.72 & 0.02 \\
\hline 6 & 27.808703 & 22.58307422 & -12.36 & 0.05 & -12.82 & 0.06 & -13.22 & 0.02 \\
\hline 7 & 27.80909168 & 22.58243561 & -12.16 & 0.02 & -12.89 & 0.06 & -11.48 & 0.10 \\
\hline 8 & 27.80945496 & 22.58181348 & -12.71 & 0.03 & -13.26 & 0.05 & -12.86 & 0.03 \\
\hline 9 & 27.80898694 & 22.58283593 & -12.19 & 0.03 & -13.14 & 0.04 & -11.33 & 0.03 \\
\hline 10 & 27.8097772 & 22.58144334 & -13.05 & 0.01 & -13.70 & 0.01 & -12.90 & 0.02 \\
\hline 11 & 27.80843983 & 22.58463146 & -10.70 & 0.07 & -11.21 & 0.08 & -11.08 & 0.14 \\
\hline 12 & 27.80929961 & 22.58314413 & -12.17 & 0.03 & -12.87 & 0.06 & -12.26 & 0.04 \\
\hline 13 & 27.8105601 & 22.5808227 & -10.89 & 0.02 & -11.31 & 0.03 & -11.60 & 0.05 \\
\hline 14 & 27.80927543 & 22.58330795 & -10.97 & 0.06 & -11.34 & 0.09 & -12.06 & 0.05 \\
\hline 15 & 27.80952221 & 22.58331847 & -11.14 & 0.05 & -11.85 & 0.13 & -12.28 & 0.04 \\
\hline 16 & 27.81139357 & 22.58329741 & -11.56 & 0.02 & -11.93 & 0.04 & -12.55 & 0.03 \\
\hline 17 & 27.80755568 & 22.58055984 & -12.43 & 0.02 & -13.46 & 0.04 & -11.70 & 0.07 \\
\hline
\end{tabular}

Table 10

Derived Properties of Star Clusters in NGC 0695

\begin{tabular}{|c|c|c|c|c|c|c|}
\hline ID & Log(Age) & $\sigma_{\mathrm{Age}}$ & $\log \left(M / M_{\odot}\right)$ & $\sigma_{M}$ & $A_{V}$ & $\sigma_{A_{V}}$ \\
\hline 1 & 6.70 & 0.92 & 6.22 & 0.76 & 1.70 & 0.84 \\
\hline 2 & 6.66 & 0.82 & 5.99 & 0.66 & 1.40 & 0.70 \\
\hline 3 & 6.40 & 0.74 & 6.05 & 0.71 & 1.90 & 0.76 \\
\hline 4 & 6.70 & 0.86 & 5.85 & 0.63 & 1.30 & 0.65 \\
\hline 5 & 6.66 & 0.40 & 6.84 & 0.17 & 1.50 & 0.42 \\
\hline 6 & 6.68 & 0.24 & 6.40 & 0.31 & 1.30 & 0.25 \\
\hline 7 & 8.56 & 0.86 & 7.14 & 0.17 & 0.01 & 0.02 \\
\hline 8 & 8.41 & 0.01 & 7.23 & 0.18 & 0.01 & 0.03 \\
\hline 9 & 8.56 & 0.03 & 7.26 & 0.21 & 0.20 & 0.10 \\
\hline 10 & 8.46 & 0.48 & 7.40 & 0.17 & 0.01 & 0.02 \\
\hline 11 & 8.36 & 0.52 & 6.39 & 0.50 & 0.01 & 0.48 \\
\hline 12 & 8.41 & 0.04 & 7.07 & 0.20 & 0.10 & 0.10 \\
\hline 13 & 6.66 & 0.76 & 5.86 & 0.64 & 1.40 & 0.67 \\
\hline 14 & 6.66 & 0.40 & 5.79 & 0.28 & 1.20 & 0.21 \\
\hline 15 & 6.74 & 0.90 & 5.83 & 0.59 & 1.10 & 0.61 \\
\hline 16 & 6.66 & 0.49 & 6.03 & 0.18 & 1.20 & 0.05 \\
\hline 17 & 8.51 & 0.04 & 7.36 & 0.22 & 0.30 & 0.13 \\
\hline
\end{tabular}


Table 11

Observed Properties of Star Clusters in UGC 02369

\begin{tabular}{|c|c|c|c|c|c|c|c|c|}
\hline ID & R.A. & Decl. & $M_{B}$ & $\sigma_{B}$ & $M_{I}$ & $\sigma_{I}$ & $M_{\mathrm{FUV}}$ & $\sigma_{\mathrm{FUV}}$ \\
\hline 1 & 43.50963156 & 14.97660266 & -10.85 & 0.06 & -11.31 & 0.13 & -12.39 & 0.02 \\
\hline 2 & 43.50342276 & 14.96966218 & -9.69 & 0.10 & -10.01 & 0.15 & -11.38 & 0.05 \\
\hline 3 & 43.5048427 & 14.97058419 & -9.36 & 0.12 & -10.28 & 0.14 & -11.80 & 0.04 \\
\hline 4 & 43.50519206 & 14.96870793 & -9.54 & 0.10 & -10.56 & 0.09 & -11.40 & 0.05 \\
\hline 5 & 43.50404048 & 14.97111855 & -12.20 & 0.01 & -12.98 & 0.01 & -11.69 & 0.04 \\
\hline 6 & 43.50533035 & 14.97014987 & -9.73 & 0.17 & -11.26 & 0.10 & -11.04 & 0.05 \\
\hline 7 & 43.50518643 & 14.9734774 & -9.84 & 0.10 & -10.55 & 0.13 & -10.86 & 0.11 \\
\hline 8 & 43.50567241 & 14.97086958 & -9.81 & 0.11 & -10.84 & 0.10 & -10.58 & 0.11 \\
\hline 9 & 43.50995014 & 14.97180788 & -10.08 & 0.07 & -11.16 & 0.06 & -11.94 & 0.03 \\
\hline
\end{tabular}

Table 12

Derived Properties of Star Clusters in UGC 02369

\begin{tabular}{|c|c|c|c|c|c|c|}
\hline ID & $\log ($ Age $)$ & $\sigma_{\mathrm{Age}}$ & $\log \left(M / M_{\odot}\right)$ & $\sigma_{M}$ & $A_{V}$ & $\sigma_{A V}$ \\
\hline 1 & 5.10 & 0.77 & 5.68 & 0.52 & 1.60 & 0.50 \\
\hline 2 & 6.36 & 0.58 & 4.99 & 0.45 & 1.40 & 0.40 \\
\hline 3 & 7.04 & 0.20 & 4.47 & 0.26 & 0.20 & 0.15 \\
\hline 4 & 7.63 & 0.26 & 4.93 & 0.26 & 0.10 & 0.17 \\
\hline 5 & 6.66 & 0.03 & 6.20 & 0.17 & 2.20 & 0.05 \\
\hline 6 & 6.92 & 0.21 & 4.98 & 0.31 & 1.10 & 0.19 \\
\hline 7 & 6.72 & 0.74 & 4.74 & 0.59 & 1.20 & 0.59 \\
\hline 8 & 7.81 & 0.55 & 5.46 & 0.51 & 0.70 & 0.49 \\
\hline 9 & 7.34 & 0.18 & 5.22 & 0.23 & 0.50 & 0.12 \\
\hline
\end{tabular}

Table 13

Observed Properties of Star Clusters in NGC 1614

\begin{tabular}{|c|c|c|c|c|c|c|c|c|}
\hline ID & R.A. & Decl. & $M_{B}$ & $\sigma_{B}$ & $M_{I}$ & $\sigma_{I}$ & $M_{\mathrm{FUV}}$ & $\sigma_{\mathrm{FUV}}$ \\
\hline 1 & 68.49833746 & -8.5836658 & -11.21 & 0.01 & -11.83 & 0.01 & -12.24 & 0.01 \\
\hline 2 & 68.49787214 & -8.579994493 & -11.99 & 0.01 & -12.46 & 0.02 & -13.06 & 0.04 \\
\hline 3 & 68.49775125 & -8.5795586 & -12.48 & 0.02 & -13.13 & 0.02 & -13.52 & 0.02 \\
\hline 4 & 68.49797702 & -8.579081625 & -13.73 & 0.01 & -14.18 & 0.01 & -15.09 & 0.02 \\
\hline 5 & 68.49796061 & -8.578745709 & -12.92 & 0.01 & -13.53 & 0.02 & -14.03 & 0.03 \\
\hline 6 & 68.49813488 & -8.578589371 & -11.62 & 0.05 & -11.90 & 0.10 & -12.76 & 0.09 \\
\hline 7 & 68.49855752 & -8.57973638 & -11.62 & 0.02 & -12.37 & 0.02 & -12.41 & 0.06 \\
\hline 8 & 68.49957784 & -8.583032955 & -10.67 & 0.02 & -10.81 & 0.03 & -12.98 & 0.04 \\
\hline 9 & 68.49815445 & -8.578312212 & -13.05 & 0.02 & -13.48 & 0.03 & -14.05 & 0.03 \\
\hline 10 & 68.49829417 & -8.578581 & -11.86 & 0.09 & -11.86 & 0.17 & -13.44 & 0.13 \\
\hline 11 & 68.49823898 & -8.578206022 & -12.72 & 0.03 & -12.49 & 0.06 & -13.38 & 0.08 \\
\hline 12 & 68.49868598 & -8.578484509 & -15.33 & 0.01 & -16.06 & 0.01 & -16.13 & 0.01 \\
\hline 13 & 68.49872601 & -8.578589212 & -12.07 & 0.10 & -11.77 & 0.11 & -12.69 & 0.16 \\
\hline 14 & 68.49882795 & -8.578206944 & -13.22 & 0.02 & -13.47 & 0.03 & -15.43 & 0.02 \\
\hline 15 & 68.4986906 & -8.577771465 & -12.76 & 0.01 & -13.38 & 0.01 & -13.75 & 0.02 \\
\hline 16 & 68.49912105 & -8.578165885 & -12.53 & 0.05 & -12.37 & 0.08 & -14.35 & 0.03 \\
\hline 17 & 68.49965474 & -8.579516099 & -14.21 & 0.01 & -15.25 & 0.01 & -13.93 & 0.02 \\
\hline 18 & 68.49930324 & -8.57824455 & -12.07 & 0.06 & -13.76 & 0.03 & -12.88 & 0.08 \\
\hline 19 & 68.49929236 & -8.578158424 & -10.73 & 0.21 & -12.65 & 0.10 & -12.70 & 0.15 \\
\hline 20 & 68.49973941 & -8.579291182 & -12.81 & 0.04 & -13.80 & 0.03 & -13.22 & 0.03 \\
\hline 21 & 68.49982001 & -8.579497527 & -11.54 & 0.11 & -12.38 & 0.07 & -12.83 & 0.05 \\
\hline 22 & 68.49952214 & -8.578509292 & -13.29 & 0.01 & -14.60 & 0.02 & -12.72 & 0.05 \\
\hline 23 & 68.49939493 & -8.578038288 & -12.50 & 0.02 & -13.18 & 0.02 & -12.83 & 0.09 \\
\hline 24 & 68.49998928 & -8.579944805 & -12.74 & 0.02 & -13.82 & 0.03 & -13.26 & 0.03 \\
\hline 25 & 68.50060521 & -8.578602704 & -13.65 & 0.01 & -14.40 & 0.02 & -13.43 & 0.03 \\
\hline
\end{tabular}


Table 14

Derived Properties of Star Clusters in NGC 1614

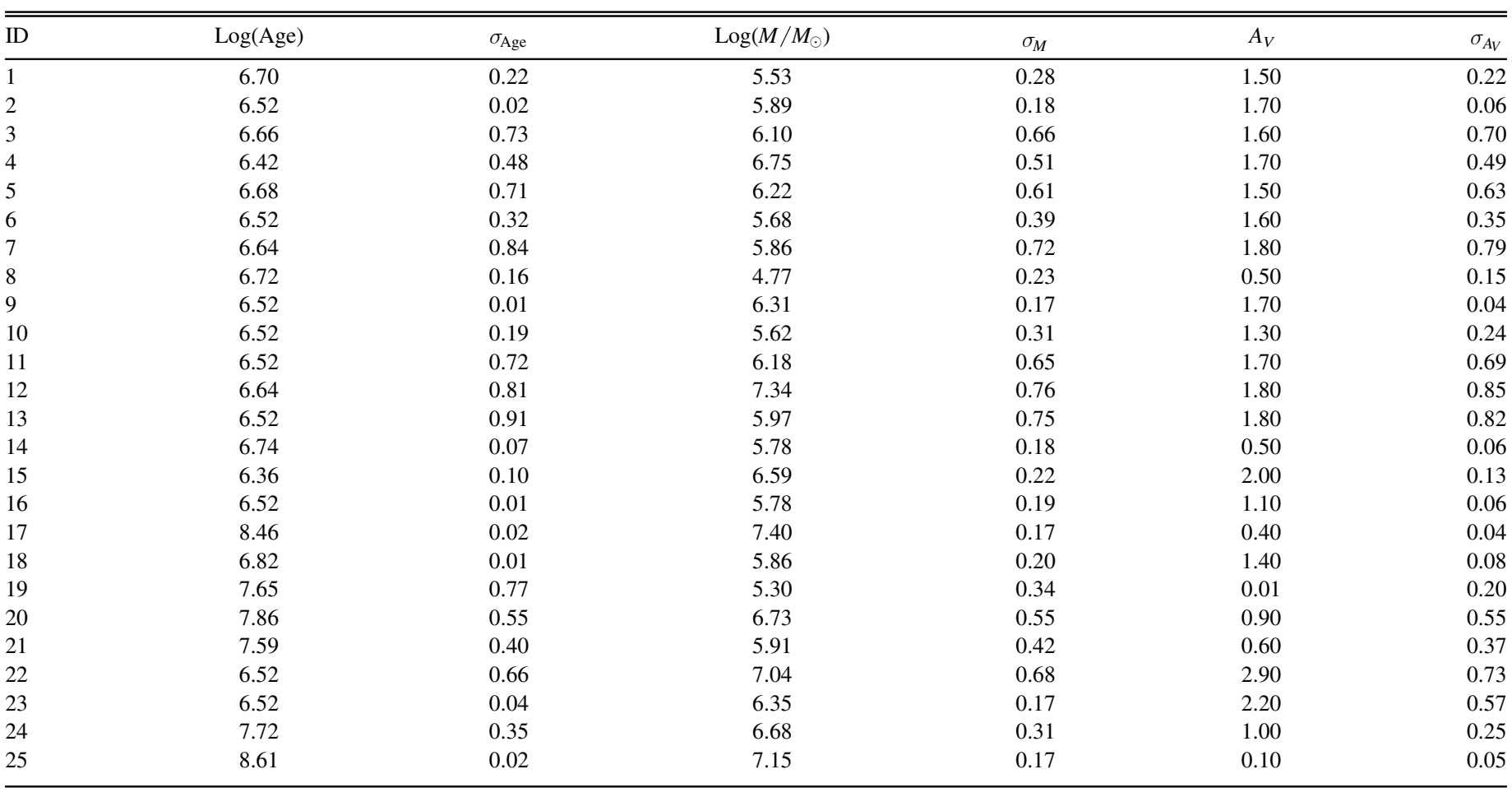

Table 15

Observed Properties of Star Clusters in 2MASX J06094582-2140234

\begin{tabular}{|c|c|c|c|c|c|c|c|c|}
\hline ID & R.A. & Decl. & $M_{B}$ & $\sigma_{B}$ & $M_{I}$ & $\sigma_{I}$ & $M_{\mathrm{FUV}}$ & $\sigma_{\mathrm{FUV}}$ \\
\hline 1 & 92.438548 & -21.67128502 & -10.07 & 0.09 & -10.29 & 0.14 & -11.05 & 0.04 \\
\hline 2 & 92.43987859 & -21.67130163 & -10.68 & 0.06 & -10.77 & 0.10 & -13.05 & 0.01 \\
\hline 3 & 92.43715675 & -21.67545341 & -11.44 & 0.02 & -11.68 & 0.04 & -13.62 & 0.02 \\
\hline 4 & 92.4409611 & -21.6715528 & -11.60 & 0.03 & -12.20 & 0.05 & -14.04 & 0.05 \\
\hline 5 & 92.4416782 & -21.67398257 & -12.05 & 0.03 & -11.92 & 0.12 & -13.73 & 0.02 \\
\hline 6 & 92.44229618 & -21.67366993 & -10.76 & 0.07 & -11.07 & 0.15 & -12.90 & 0.01 \\
\hline 7 & 92.44270943 & -21.67433539 & -10.88 & 0.06 & -10.84 & 0.18 & -10.91 & 0.03 \\
\hline 8 & 92.44164311 & -21.67543932 & -14.49 & 0.03 & -14.79 & 0.09 & -14.68 & 0.05 \\
\hline 9 & 92.44054901 & -21.67659996 & -10.76 & 0.05 & -11.01 & 0.10 & -11.59 & 0.04 \\
\hline 10 & 92.44310457 & -21.67562528 & -11.02 & 0.07 & -11.81 & 0.07 & -12.66 & 0.01 \\
\hline
\end{tabular}

Table 16

Derived Properties of Star Clusters in 2MASX J06094582-2140234

\begin{tabular}{|c|c|c|c|c|c|c|}
\hline ID & $\log ($ Age $)$ & $\sigma_{\mathrm{Age}}$ & $\log \left(M / M_{\odot}\right)$ & $\sigma_{M}$ & $A_{V}$ & $\sigma_{A_{V}}$ \\
\hline 1 & 6.74 & 0.09 & 4.79 & 0.20 & 1.00 & 0.08 \\
\hline 2 & 6.72 & 0.29 & 4.78 & 0.30 & 0.50 & 0.24 \\
\hline 3 & 6.74 & 0.03 & 5.07 & 0.19 & 0.50 & 0.09 \\
\hline 4 & 6.92 & 0.05 & 5.14 & 0.17 & 0.20 & 0.04 \\
\hline 5 & 6.70 & 0.08 & 5.55 & 0.17 & 0.90 & 0.05 \\
\hline 6 & 6.74 & 0.36 & 4.85 & 0.35 & 0.60 & 0.30 \\
\hline 7 & 7.91 & 0.03 & 6.04 & 0.17 & 1.00 & 0.29 \\
\hline 8 & 7.91 & 0.03 & 7.48 & 0.16 & 1.00 & 0.20 \\
\hline 9 & 7.16 & 0.09 & 5.52 & 0.18 & 1.00 & 0.07 \\
\hline 10 & 7.36 & 0.31 & 5.55 & 0.28 & 0.60 & 0.21 \\
\hline
\end{tabular}


Table 17

Observed Properties of Star Clusters in 2MASX J08370182-4954302

\begin{tabular}{lccccccc}
\hline \hline ID & R.A. & Decl. & $M_{B}$ & $\sigma_{B}$ & $M_{I}$ & $\sigma_{I}$ & $M_{\text {FUV }}$ \\
\hline 1 & 129.2557186 & -49.90846967 & -14.83 & 0.04 & -15.31 & 0.03 & -17.28 \\
2 & 129.2561197 & -49.90840169 & -15.24 & 0.05 & -15.31 & 0.03 & -17.63 \\
3 & 129.2561534 & -49.90828131 & -15.85 & 0.02 & -15.68 & 0.02 & -18.76 \\
4 & 129.2574869 & -49.90904604 & -15.42 & 0.01 & -15.36 & 0.02 & -17.47 \\
5 & 129.2571648 & -49.90869922 & -18.38 & 0.01 & -18.23 & 0.01 & -21.39 \\
6 & 129.2571314 & -49.90851337 & -19.79 & 0.01 & -19.10 & 0.01 & 0.01 \\
\hline
\end{tabular}

Table 18

Derived Properties of Star Clusters in 2MASX J08370182-4954302

\begin{tabular}{lcccccc}
\hline \hline ID & $\log ($ Age $)$ & $\sigma_{\text {Age }}$ & $\log \left(M / M_{\odot}\right)$ & $\sigma_{M}$ & $A_{V}$ \\
\hline 1 & 6.86 & 0.02 & 6.38 & 0.18 & 0.20 \\
2 & 5.10 & 0.64 & 7.12 & 0.43 & 0.00 \\
3 & 5.10 & 0.46 & 7.15 & 0.17 & 0.05 \\
4 & 6.66 & 0.03 & 6.59 & 0.16 & 0.17 \\
5 & 5.10 & 0.41 & 8.17 & 0.17 & 0.02 \\
6 & 6.66 & 0.61 & 8.07 & 0.16 & 0.00 \\
\end{tabular}

Table 19

Observed Properties of Star Clusters in NGC 2623

\begin{tabular}{llllllrr}
\hline \hline ID & \multicolumn{1}{c}{ R.A. } & \multicolumn{1}{c}{ Decl. } & $M_{B}$ & $\sigma_{B}$ & $M_{I}$ & $\sigma_{I}$ & $M_{\mathrm{FUV}}$ \\
\hline 1 & 129.5980304 & 25.75459514 & -14.04 & 0.01 & -14.98 & 0.01 & -13.25 \\
2 & 129.5990031 & 25.7538954 & -11.45 & 0.02 & -11.94 & 0.05 & -12.53 \\
3 & 129.5994079 & 25.75382853 & -11.39 & 0.02 & -11.90 & 0.05 & -12.41 \\
4 & 129.5990874 & 25.751723 & -11.53 & 0.01 & -12.08 & 0.01 & -11.79 \\
5 & 129.5995056 & 25.75209619 & -10.61 & 0.02 & -10.88 & 0.03 & -11.91 \\
6 & 129.599766 & 25.75096143 & -11.27 & 0.01 & -11.85 & 0.01 & -11.47 \\
7 & 129.5999493 & 25.75179613 & -13.15 & 0.01 & -13.82 & 0.01 & -12.75 \\
8 & 129.6003718 & 25.75134324 & -11.08 & 0.02 & -11.09 & 0.05 & -12.93 \\
9 & 129.6010618 & 25.75100371 & -11.58 & 0.01 & -12.06 & 0.02 & 0.03 \\
10 & 129.6013548 & 25.75053983 & -10.88 & 0.03 & -11.40 & 0.03 & -12.89 \\
11 & 129.601522 & 25.75112441 & -11.24 & 0.02 & -11.68 & 0.03 & -11.30 \\
& & & & & & 0.03 & -11.90 \\
\hline
\end{tabular}

Table 20

Derived Properties of Star Clusters in NGC 2623

\begin{tabular}{|c|c|c|c|c|c|c|}
\hline ID & $\log ($ Age $)$ & $\sigma_{\mathrm{Age}}$ & $\log \left(M / M_{\odot}\right)$ & $\sigma_{M}$ & $A_{V}$ & $\sigma_{A_{V}}$ \\
\hline 1 & 8.56 & 0.18 & 7.33 & 0.16 & 0.10 & 0.02 \\
\hline 2 & 6.54 & 0.56 & 5.48 & 0.50 & 1.50 & 0.49 \\
\hline 3 & 6.62 & 0.47 & 5.34 & 0.43 & 1.30 & 0.40 \\
\hline 4 & 8.36 & 0.64 & 6.16 & 0.16 & 0.10 & 0.03 \\
\hline 5 & 6.66 & 0.03 & 4.93 & 0.19 & 1.00 & 0.09 \\
\hline 6 & 6.68 & 0.72 & 5.46 & 0.16 & 1.50 & 0.27 \\
\hline 7 & 7.86 & 0.67 & 7.25 & 0.16 & 1.50 & 0.29 \\
\hline 8 & 6.66 & 0.05 & 4.90 & 0.21 & 0.60 & 0.12 \\
\hline 9 & 6.70 & 0.57 & 5.32 & 0.47 & 1.00 & 0.45 \\
\hline 10 & 6.68 & 0.02 & 5.30 & 0.17 & 1.50 & 0.03 \\
\hline 11 & 6.66 & 0.72 & 5.44 & 0.60 & 1.50 & 0.62 \\
\hline
\end{tabular}


Table 21

Observed Properties of Star Clusters in UGC 04881

\begin{tabular}{lccccccrc}
\hline \hline ID & R.A. & Decl. & $M_{B}$ & $\sigma_{B}$ & $M_{I}$ & $\sigma_{I}$ & $M_{\mathrm{FUV}}$ \\
\hline 1 & 138.9806254 & 44.33224681 & -13.41 & 0.02 & -14.51 & 0.05 & -11.65 \\
2 & 138.9802968 & 44.33445408 & -12.38 & 0.02 & -13.38 & 0.01 & -11.12 \\
3 & 138.9841104 & 44.33189026 & -13.48 & 0.01 & -14.72 & 0.01 & -11.29 \\
4 & 138.9857806 & 44.32941604 & -11.38 & 0.08 & -12.28 & 0.11 & -11.33 \\
5 & 138.9861709 & 44.32809268 & -10.97 & 0.13 & -11.15 & 0.20 & -12.59 \\
\hline
\end{tabular}

Table 22

Derived Properties of Star Clusters in UGC 04881

\begin{tabular}{lcccccc}
\hline \hline ID & $\log ($ Age $)$ & $\sigma_{\text {Age }}$ & $\log \left(M / M_{\odot}\right)$ & $\sigma_{M}$ & $A_{V}$ \\
\hline 1 & 8.71 & 0.03 & 7.17 & 0.23 & 0.01 & 1.90 \\
2 & 8.06 & 0.02 & 7.27 & 0.19 & 0.16 \\
3 & 8.71 & 0.10 & 7.36 & 0.53 & 0.09 \\
4 & 0.44 & 5.71 & 0.42 & 1.90 \\
5 & 6.68 & 0.36 & 4.97 & 0.82 & 0.52 \\
\hline
\end{tabular}

Table 23

Observed Properties of Star Clusters in IC 2545

\begin{tabular}{|c|c|c|c|c|c|c|c|c|}
\hline$\overline{\mathrm{ID}}$ & R.A. & Decl. & $M_{B}$ & $\sigma_{B}$ & $M_{I}$ & $\sigma_{I}$ & $M_{\mathrm{FUV}}$ & $\sigma_{\mathrm{FUV}}$ \\
\hline 1 & 151.5136356 & -33.88528106 & -10.23 & 0.08 & -11.06 & 0.08 & -11.36 & $\overline{0.11}$ \\
\hline 2 & 151.5136616 & -33.88695096 & -10.55 & 0.07 & -10.99 & 0.08 & -11.95 & 0.11 \\
\hline 3 & 151.5140689 & -33.88785616 & -10.73 & 0.07 & -11.43 & 0.06 & -11.72 & 0.13 \\
\hline 4 & 151.5146406 & -33.88747231 & -9.72 & 0.13 & -10.15 & 0.15 & -11.22 & 0.26 \\
\hline 5 & 151.5148205 & -33.8873956 & -10.48 & 0.09 & -10.74 & 0.13 & -11.64 & 0.17 \\
\hline 6 & 151.515442 & -33.88795458 & -11.40 & 0.07 & -11.61 & 0.08 & -13.21 & 0.17 \\
\hline 7 & 151.5153282 & -33.88941777 & -11.77 & 0.03 & -12.68 & 0.03 & -12.06 & 0.36 \\
\hline 8 & 151.5152205 & -33.89096417 & -11.67 & 0.03 & -11.46 & 0.05 & -13.28 & 0.05 \\
\hline 9 & 151.5155505 & -33.88790446 & -10.54 & 0.11 & -10.86 & 0.19 & -12.31 & 0.07 \\
\hline 10 & 151.5161603 & -33.88389939 & -10.85 & 0.04 & -11.52 & 0.04 & -11.83 & 0.22 \\
\hline 11 & 151.5172199 & -33.88393251 & -11.68 & 0.02 & -12.21 & 0.03 & -12.54 & 0.21 \\
\hline 12 & 151.5168118 & -33.8905397 & -10.84 & 0.04 & -10.95 & 0.07 & -12.44 & 0.29 \\
\hline 13 & 151.5177074 & -33.88509063 & -14.32 & 0.01 & -15.84 & 0.01 & -14.21 & 0.04 \\
\hline 14 & 151.517576 & -33.88570119 & -10.70 & 0.20 & -11.73 & 0.19 & -11.88 & 0.36 \\
\hline 15 & 151.5177403 & -33.8853364 & -14.65 & 0.02 & -15.38 & 0.02 & -14.71 & 0.03 \\
\hline 16 & 151.5199867 & -33.88613476 & -9.91 & 0.19 & -11.22 & 0.07 & -12.03 & 0.01 \\
\hline 17 & 151.5202629 & -33.88460375 & -12.84 & 0.03 & -13.94 & 0.02 & -12.17 & 0.06 \\
\hline 18 & 151.5205798 & -33.88546044 & -11.59 & 0.04 & -12.21 & 0.08 & -12.04 & 0.27 \\
\hline 19 & 151.5210485 & -33.88549176 & -11.29 & 0.04 & -11.50 & 0.08 & -11.79 & 0.24 \\
\hline
\end{tabular}

\section{NGC 0695}

NGC 0695 is a face-on spiral galaxy with a companion at a projected nuclear separation of $\sim 26^{\prime \prime}(16 \mathrm{kpc})$ to the northwest. There are multiple spiral arms on the northwestern half of the galaxy, and star clusters are distributed throughout disk (Figure 13). The maximum $A_{V}$ adopted for this galaxy is 2.8 mag of visual extinction (see Figure 14; Kennicutt et al. 2009). The observed and derived properties for the star clusters identified in this galaxy are given in Tables 9 and 10, respectively.

\section{UGC 02369}

UGC 02369 is a mid-stage merger consisting of a southern face-on galaxy (MCG +02-08-029) and an inclined northern galaxy (MCG +02-08-030). The nuclei of the two galaxies are separated by $\sim 21^{\prime \prime}(13 \mathrm{kpc})$. A spiral arm containing multiple star clusters extends from the nucleus of the southern galaxy toward the northern galaxy (Figure 15). The maximum $A_{V}$ adopted for this galaxy is $2.3 \mathrm{mag}$ of visual extinction (see Figure 16; van Driel et al. 2001). The observed and derived properties for the star clusters identified in this galaxy are given in Tables 11 and 12, respectively.

\section{NGC 1614}

NGC 1614 is a late-stage merger with two resolved components in the nucleus separated by $\sim 0$ ". 8 (300 pc). Beyond the nucleus are two well-defined spiral arms, with a significant number of bright clusters scattered throughout this region Figure 17. The maximum $A_{V}$ adopted for this galaxy is $4.0 \mathrm{mag}$ of visual extinction (see Figure 18; Alonso-Herrero et al. 2001). The observed and derived 
Table 24

Derived Properties of Star Clusters in IC 2545

\begin{tabular}{|c|c|c|c|c|c|c|}
\hline ID & Log(Age) & $\sigma_{\text {Age }}$ & $\log \left(M / M_{\odot}\right)$ & $\sigma_{M}$ & $A_{V}$ & $\sigma_{A_{V}}$ \\
\hline 1 & 6.78 & 0.03 & 4.88 & 0.18 & 1.10 & 0.29 \\
\hline 2 & 6.34 & 0.40 & 5.47 & 0.18 & 1.60 & 0.46 \\
\hline 3 & 8.06 & 0.54 & 5.71 & 0.18 & 0.20 & 0.19 \\
\hline 4 & 5.10 & 0.35 & 5.23 & 0.21 & 1.60 & 0.26 \\
\hline 5 & 6.66 & 0.21 & 4.93 & 0.19 & 1.10 & 0.10 \\
\hline 6 & 6.46 & 0.33 & 5.58 & 0.18 & 1.20 & 0.35 \\
\hline 7 & 6.46 & 0.17 & 6.31 & 0.17 & 2.30 & 0.51 \\
\hline 8 & 6.66 & 0.10 & 5.19 & 0.17 & 0.70 & 0.07 \\
\hline 9 & 6.00 & 0.31 & 5.35 & 0.20 & 1.40 & 0.56 \\
\hline 10 & 8.11 & 0.31 & 5.73 & 0.17 & 0.10 & 0.55 \\
\hline 11 & 6.64 & 0.57 & 5.54 & 0.17 & 1.40 & 0.41 \\
\hline 12 & 6.66 & 0.11 & 4.91 & 0.17 & 0.80 & 0.58 \\
\hline 13 & 7.74 & 0.13 & 7.60 & 0.17 & 1.40 & 0.41 \\
\hline 14 & 7.63 & 0.07 & 5.66 & 0.27 & 0.60 & 0.08 \\
\hline 15 & 6.66 & 0.59 & 7.02 & 0.17 & 1.90 & 0.10 \\
\hline 16 & 5.10 & 0.36 & 6.58 & 0.26 & 4.00 & 0.06 \\
\hline 17 & 8.41 & 0.38 & 6.99 & 0.17 & 0.60 & 0.28 \\
\hline 18 & 8.31 & 0.26 & 6.16 & 0.17 & 0.10 & 0.54 \\
\hline 19 & 6.66 & 0.50 & 5.46 & 0.17 & 1.50 & 0.22 \\
\hline
\end{tabular}

Table 25

Observed Properties of Star Clusters in NGC 3256

\begin{tabular}{|c|c|c|c|c|c|c|c|c|}
\hline ID & R.A. & Decl. & $M_{B}$ & $\sigma_{B}$ & $M_{I}$ & $\sigma_{I}$ & $M_{\mathrm{FUV}}$ & $\sigma_{\mathrm{FUV}}$ \\
\hline 1 & 156.9608449 & -43.9024964 & -12.20 & 0.02 & -12.93 & 0.03 & -12.65 & 0.01 \\
\hline 2 & 156.9612737 & -43.90178128 & -12.75 & 0.01 & -12.90 & 0.01 & -14.09 & 0.01 \\
\hline 3 & 156.9630369 & -43.90314637 & -12.96 & 0.01 & -13.87 & 0.01 & -12.18 & 0.02 \\
\hline 4 & 156.9636042 & -43.9045422 & -14.02 & 0.01 & -14.82 & 0.01 & -12.97 & 0.02 \\
\hline 5 & 156.9634535 & -43.90288363 & -12.88 & 0.02 & -14.04 & 0.02 & -12.02 & 0.05 \\
\hline 6 & 156.9635947 & -43.90369683 & -13.70 & 0.02 & -15.00 & 0.01 & -12.74 & 0.01 \\
\hline 7 & 156.9633212 & -43.89955977 & -12.29 & 0.02 & -13.32 & 0.02 & -12.79 & 0.02 \\
\hline 8 & 156.9634618 & -43.89962047 & -11.36 & 0.01 & -12.37 & 0.02 & -12.10 & 0.04 \\
\hline 9 & 156.9640714 & -43.90271444 & -13.34 & 0.02 & -14.31 & 0.01 & -13.43 & 0.04 \\
\hline 10 & 156.9646123 & -43.90392525 & -13.49 & 0.03 & -13.98 & 0.03 & -14.25 & 0.01 \\
\hline 11 & 156.9652121 & -43.90415987 & -13.56 & 0.02 & -13.33 & 0.03 & -14.86 & 0.01 \\
\hline 12 & 156.9653068 & -43.90414225 & -13.36 & 0.02 & -13.27 & 0.04 & -13.90 & 0.02 \\
\hline 13 & 156.9655228 & -43.9046008 & -12.70 & 0.01 & -13.66 & 0.01 & -12.20 & 0.02 \\
\hline 14 & 156.9654982 & -43.90351402 & -13.21 & 0.01 & -14.42 & 0.01 & -11.32 & 0.05 \\
\hline 15 & 156.966498 & -43.90415946 & -13.52 & 0.01 & -14.61 & 0.01 & -12.95 & 0.01 \\
\hline 16 & 156.9666609 & -43.90272713 & -13.74 & 0.01 & -13.96 & 0.01 & -15.46 & 0.01 \\
\hline 17 & 156.9665347 & -43.90083852 & -10.75 & 0.01 & -11.16 & 0.01 & -11.76 & 0.03 \\
\hline 18 & 156.9667894 & -43.90232476 & -10.09 & 0.05 & -10.93 & 0.05 & -12.02 & 0.02 \\
\hline 19 & 156.9675522 & -43.9029773 & -12.70 & 0.01 & -13.55 & 0.01 & -13.15 & 0.01 \\
\hline
\end{tabular}

properties for the star clusters identified in this galaxy are given in Tables 13 and 14, respectively.

\section{MASX J06094582-2140234}

2MASX J06094582-2140234 is a mid-stage merger consisting of two face-on galaxies which appear to overlap and have a projected separation of $\sim 8$ ". $4(6.3 \mathrm{kpc})$. Prominent rings/arms in each galaxy contain the bulk of the visible star clusters (Figure 19). The maximum $A_{V}$ adopted for this galaxy is 1.0 mag of visual extinction (see Figure 20; Miralles-Caballero et al. 2012). The observed and derived properties for the star clusters identified in this galaxy are given in Tables 15 and 16, respectively.

\section{MASX J08370182-4954302}

2MASX J08370182-4954302 is a mid-stage merger containing two nuclei separated by $\sim 0$ ". $66(0.36 \mathrm{kpc})$. Surrounding the nuclei are multiple bright star clusters in a spiral ridge just northwest and west of the nuclei (Figure 21). The maximum $A_{V}$ adopted for this galaxy is $3.7 \mathrm{mag}$ of visual extinction (see Figure 22; Rich et al. 2012). The observed and derived 
Table 26

Derived Properties of Star Clusters in NGC 3256

\begin{tabular}{|c|c|c|c|c|c|c|}
\hline ID & $\log ($ Age $)$ & $\sigma_{\mathrm{Age}}$ & $\log \left(M / M_{\odot}\right)$ & $\sigma_{M}$ & $A_{V}$ & $\sigma_{A_{V}}$ \\
\hline 1 & 6.68 & 0.03 & 5.88 & 0.18 & 1.60 & 0.06 \\
\hline 2 & 6.66 & 0.12 & 5.78 & 0.17 & 1.00 & 0.27 \\
\hline 3 & 8.36 & 0.19 & 7.10 & 0.17 & 0.80 & 0.07 \\
\hline 4 & 8.41 & 0.10 & 7.56 & 0.17 & 0.80 & 0.30 \\
\hline 5 & 8.36 & 0.03 & 7.13 & 0.18 & 0.90 & 0.06 \\
\hline 6 & 8.36 & 0.03 & 7.51 & 0.20 & 1.00 & 0.11 \\
\hline 7 & 7.86 & 0.54 & 6.54 & 0.42 & 0.80 & 0.38 \\
\hline 8 & 7.74 & 0.02 & 6.10 & 0.18 & 0.80 & 0.06 \\
\hline 9 & 6.54 & 0.13 & 6.61 & 0.27 & 2.20 & 0.20 \\
\hline 10 & 6.66 & 0.01 & 6.29 & 0.17 & 1.40 & 0.03 \\
\hline 11 & 6.66 & 0.47 & 6.06 & 0.17 & 0.90 & 0.02 \\
\hline 12 & 6.66 & 0.22 & 6.24 & 0.17 & 1.40 & 0.03 \\
\hline 13 & 8.31 & 0.02 & 6.97 & 0.17 & 0.80 & 0.03 \\
\hline 14 & 8.56 & 0.03 & 7.37 & 0.17 & 0.80 & 0.02 \\
\hline 15 & 8.31 & 0.10 & 7.30 & 0.17 & 0.80 & 0.08 \\
\hline 16 & 6.52 & 0.07 & 6.19 & 0.26 & 1.10 & 0.19 \\
\hline 17 & 6.68 & 0.01 & 5.09 & 0.18 & 1.20 & 0.05 \\
\hline 18 & 6.86 & 0.03 & 4.80 & 0.17 & 0.80 & 0.37 \\
\hline 19 & 6.44 & 0.14 & 6.52 & 0.25 & 2.20 & 0.17 \\
\hline
\end{tabular}

Table 27

Observed Properties of Star Clusters in Arp 148

\begin{tabular}{|c|c|c|c|c|c|c|c|c|}
\hline ID & R.A. & Decl. & $M_{B}$ & $\sigma_{B}$ & $M_{I}$ & $\sigma_{I}$ & $M_{\mathrm{FUV}}$ & $\sigma_{\mathrm{FUV}}$ \\
\hline 1 & 165.968436 & 40.8519488 & -12.21 & 0.01 & -12.34 & 0.02 & -14.41 & 0.02 \\
\hline 2 & 165.9678663 & 40.8505602 & -11.09 & 0.05 & -11.12 & 0.10 & -13.18 & 0.07 \\
\hline 3 & 165.9683844 & 40.85145549 & -11.10 & 0.03 & -11.36 & 0.05 & -12.86 & 0.09 \\
\hline 4 & 165.9691046 & 40.85164919 & -11.30 & 0.03 & -11.90 & 0.04 & -12.20 & 0.10 \\
\hline 5 & 165.9690976 & 40.85147869 & -11.19 & 0.03 & -12.17 & 0.03 & -13.09 & 0.09 \\
\hline 6 & 165.967872 & 40.84958083 & -10.44 & 0.07 & -10.45 & 0.13 & -12.84 & 0.09 \\
\hline 7 & 165.9678992 & 40.84893317 & -9.78 & 0.14 & -11.31 & 0.08 & -12.44 & 0.14 \\
\hline 8 & 165.9680004 & 40.84887677 & -11.27 & 0.05 & -11.84 & 0.07 & -13.06 & 0.08 \\
\hline 9 & 165.9682628 & 40.84916472 & -10.83 & 0.07 & -11.39 & 0.08 & -12.25 & 0.16 \\
\hline 10 & 165.9677623 & 40.84910158 & -11.64 & 0.03 & -11.53 & 0.07 & -14.10 & 0.03 \\
\hline 11 & 165.9704139 & 40.85187208 & -10.71 & 0.07 & -10.55 & 0.18 & -12.74 & 0.10 \\
\hline 12 & 165.968608 & 40.84903015 & -11.16 & 0.06 & -11.64 & 0.09 & -13.54 & 0.05 \\
\hline 13 & 165.967709 & 40.84993148 & -11.37 & 0.03 & -11.55 & 0.05 & -13.09 & 0.08 \\
\hline 14 & 165.9682107 & 40.84793431 & -10.78 & 0.11 & -11.78 & 0.09 & -12.22 & 0.16 \\
\hline 15 & 165.9684927 & 40.8490672 & -11.59 & 0.05 & -11.93 & 0.08 & -13.89 & 0.04 \\
\hline 16 & 165.9687209 & 40.84766184 & -12.23 & 0.03 & -12.82 & 0.07 & -13.60 & 0.05 \\
\hline 17 & 165.9720438 & 40.85223589 & -10.78 & 0.04 & -11.57 & 0.04 & -12.81 & 0.10 \\
\hline 18 & 165.969655 & 40.85013869 & -9.45 & 0.12 & -10.67 & 0.10 & -11.04 & 0.13 \\
\hline 19 & 165.9692811 & 40.84792405 & -13.17 & 0.02 & -13.56 & 0.04 & -14.63 & 0.02 \\
\hline 20 & 165.9724453 & 40.852421 & -9.47 & 0.14 & -10.32 & 0.14 & -11.32 & 0.01 \\
\hline 21 & 165.9724057 & 40.85141491 & -11.51 & 0.02 & -11.70 & 0.04 & -13.66 & 0.07 \\
\hline 22 & 165.9716475 & 40.84859071 & -11.98 & 0.02 & -12.56 & 0.03 & -12.98 & 0.09 \\
\hline 23 & 165.9715595 & 40.84827508 & -11.99 & 0.03 & -12.91 & 0.04 & -12.72 & 0.11 \\
\hline 24 & 165.9706662 & 40.84783769 & -10.84 & 0.04 & -10.45 & 0.16 & -13.07 & 0.08 \\
\hline 25 & 165.9729915 & 40.85207186 & -10.18 & 0.12 & -11.13 & 0.11 & -12.37 & 0.14 \\
\hline 26 & 165.9748241 & 40.85035121 & -12.83 & 0.04 & -14.33 & 0.09 & -14.00 & 0.03 \\
\hline
\end{tabular}

properties for the star clusters identified in this galaxy are given in Tables 17 and 18, respectively.

\section{NGC 2623}

Evans et al. (2008) discuss the detailed morphology of this galaxy at length. NGC 2623 is a late-stage merger with dust lanes running along its tidal tails into the nucleus. Several bright clusters are distributed throughout the bulge and in a "pie-wedge" concentration south of the nucleus (Figure 23). The maximum $A_{V}$ adopted for this galaxy is 1.9 mag of visual extinction (see Figure 24; Privon et al. 2013). The observed and derived properties for the star clusters identified in this galaxy are given in Tables 19 and 20, respectively. 
Table 28

Derived Properties of Star Clusters in Arp 148

\begin{tabular}{|c|c|c|c|c|c|c|}
\hline ID & $\log ($ Age $)$ & $\sigma_{\text {Age }}$ & $\log \left(M / M_{\odot}\right)$ & $\sigma_{M}$ & $A_{V}$ & $\sigma_{A_{V}}$ \\
\hline 1 & 5.10 & 0.67 & 5.96 & 0.39 & 1.10 & 0.35 \\
\hline 2 & 6.56 & 0.42 & 4.91 & 0.36 & 0.70 & 0.32 \\
\hline 3 & 6.70 & 0.51 & 4.97 & 0.34 & 0.70 & 0.29 \\
\hline 4 & 6.60 & 0.56 & 5.35 & 0.40 & 1.40 & 0.36 \\
\hline 5 & 7.12 & 0.18 & 5.44 & 0.19 & 0.50 & 0.10 \\
\hline 6 & 6.36 & 0.36 & 5.03 & 0.34 & 0.90 & 0.29 \\
\hline 7 & 5.10 & 0.01 & 5.52 & 0.27 & 2.10 & 0.15 \\
\hline 8 & 6.76 & 0.49 & 5.02 & 0.35 & 0.60 & 0.30 \\
\hline 9 & 6.74 & 0.79 & 4.99 & 0.55 & 0.90 & 0.55 \\
\hline 10 & 6.60 & 0.57 & 4.90 & 0.36 & 0.30 & 0.32 \\
\hline 11 & 6.66 & 0.11 & 4.70 & 0.28 & 0.50 & 0.20 \\
\hline 12 & 6.86 & 0.23 & 4.91 & 0.20 & 0.20 & 0.10 \\
\hline 13 & 6.52 & 0.18 & 5.25 & 0.31 & 1.10 & 0.26 \\
\hline 14 & 7.63 & 0.31 & 5.58 & 0.33 & 0.40 & 0.26 \\
\hline 15 & 6.76 & 0.05 & 4.98 & 0.18 & 0.30 & 0.07 \\
\hline 16 & 6.72 & 0.70 & 5.59 & 0.58 & 1.00 & 0.60 \\
\hline 17 & 7.49 & 0.20 & 5.42 & 0.21 & 0.20 & 0.13 \\
\hline 18 & 7.34 & 0.24 & 5.07 & 0.27 & 0.70 & 0.18 \\
\hline 19 & 6.42 & 0.22 & 6.39 & 0.33 & 1.50 & 0.28 \\
\hline 20 & 7.52 & 0.34 & 4.97 & 0.31 & 0.30 & 0.22 \\
\hline 21 & 6.72 & 0.32 & 4.99 & 0.35 & 0.40 & 0.30 \\
\hline 22 & 6.46 & 0.46 & 6.13 & 0.50 & 1.80 & 0.49 \\
\hline 23 & 6.74 & 0.45 & 5.72 & 0.38 & 1.40 & 0.34 \\
\hline 24 & 6.66 & 0.03 & 4.65 & 0.21 & 0.30 & 0.12 \\
\hline 25 & 7.30 & 0.23 & 5.13 & 0.27 & 0.30 & 0.17 \\
\hline 26 & 7.06 & 0.17 & 6.35 & 0.20 & 1.10 & 0.11 \\
\hline
\end{tabular}

Table 29

Observed Properties of Star Clusters in NGC 3690E

\begin{tabular}{|c|c|c|c|c|c|c|c|c|}
\hline ID & R.A. & Decl. & $M_{B}$ & $\sigma_{B}$ & $M_{I}$ & $\sigma_{I}$ & $M_{\mathrm{FUV}}$ & $\sigma_{\mathrm{FUV}}$ \\
\hline 1 & 172.1336515 & 58.56572969 & -11.56 & 0.01 & -12.23 & 0.01 & -11.95 & 0.02 \\
\hline 2 & 172.1424578 & 58.56116578 & -11.76 & 0.01 & -12.83 & 0.02 & -13.14 & 0.01 \\
\hline 3 & 172.1388005 & 58.56124188 & -10.20 & 0.05 & -11.19 & 0.04 & -11.52 & 0.05 \\
\hline 4 & 172.1400284 & 58.56149757 & -10.81 & 0.04 & -10.97 & 0.14 & -12.79 & 0.02 \\
\hline 5 & 172.1345803 & 58.56118873 & -8.96 & 0.04 & -9.81 & 0.05 & -11.46 & 0.03 \\
\hline 6 & 172.1392395 & 58.56156972 & -12.52 & 0.02 & -14.15 & 0.01 & -13.50 & 0.02 \\
\hline 7 & 172.1399479 & 58.56161791 & -11.73 & 0.02 & -12.28 & 0.02 & -13.31 & 0.02 \\
\hline 8 & 172.1386985 & 58.56153798 & -10.07 & 0.08 & -10.97 & 0.10 & -11.61 & 0.10 \\
\hline 9 & 172.1378106 & 58.56146996 & -10.01 & 0.04 & -11.00 & 0.04 & -11.91 & 0.04 \\
\hline 10 & 172.1387899 & 58.56177443 & -12.85 & 0.02 & -13.61 & 0.03 & -14.51 & 0.02 \\
\hline 11 & 172.1385677 & 58.56177224 & -12.15 & 0.03 & -12.98 & 0.03 & -13.82 & 0.02 \\
\hline 12 & 172.1376673 & 58.5618607 & -11.11 & 0.03 & -11.81 & 0.03 & -12.38 & 0.04 \\
\hline 13 & 172.138187 & 58.56197966 & -11.39 & 0.05 & -12.33 & 0.07 & -13.14 & 0.05 \\
\hline 14 & 172.1366287 & 58.56197706 & -10.22 & 0.18 & -12.26 & 0.06 & -11.91 & 0.06 \\
\hline 15 & 172.1365065 & 58.56198534 & -10.79 & 0.10 & -12.11 & 0.05 & -11.93 & 0.05 \\
\hline 16 & 172.1367658 & 58.56209285 & -10.89 & 0.04 & -11.16 & 0.06 & -12.39 & 0.04 \\
\hline 17 & 172.1376122 & 58.56226713 & -10.54 & 0.10 & -11.22 & 0.08 & -12.52 & 0.05 \\
\hline 18 & 172.1357033 & 58.56256555 & -9.68 & 0.08 & -9.93 & 0.10 & -11.77 & 0.02 \\
\hline 19 & 172.1349188 & 58.56268913 & -11.06 & 0.01 & -11.80 & 0.02 & -12.68 & 0.01 \\
\hline 20 & 172.1390305 & 58.56385382 & -11.61 & 0.02 & -12.65 & 0.02 & -12.09 & 0.02 \\
\hline 21 & 172.1412556 & 58.56431432 & -11.46 & 0.04 & -12.81 & 0.02 & -11.69 & 0.04 \\
\hline 22 & 172.1412392 & 58.56451117 & -11.52 & 0.02 & -12.28 & 0.03 & -12.36 & 0.01 \\
\hline 23 & 172.1433592 & 58.56475551 & -11.38 & 0.01 & -11.95 & 0.01 & -12.46 & 0.01 \\
\hline
\end{tabular}


Table 30

Derived Properties of Star Clusters in NGC 3690E

\begin{tabular}{|c|c|c|c|c|c|c|}
\hline ID & $\log ($ Age $)$ & $\sigma_{\mathrm{Age}}$ & $\log \left(M / M_{\odot}\right)$ & $\sigma_{M}$ & $A_{V}$ & $\sigma_{A V}$ \\
\hline 1 & 8.46 & 0.01 & 6.13 & 0.17 & 0.01 & 0.15 \\
\hline 2 & 6.94 & 0.05 & 5.66 & 0.17 & 1.00 & 0.35 \\
\hline 3 & 7.00 & 0.23 & 5.22 & 0.24 & 1.10 & 0.15 \\
\hline 4 & 6.56 & 0.43 & 5.06 & 0.42 & 1.00 & 0.38 \\
\hline 5 & 6.82 & 0.01 & 3.99 & 0.19 & 0.20 & 0.06 \\
\hline 6 & 6.82 & 0.83 & 5.99 & 0.18 & 1.30 & 0.04 \\
\hline 7 & 7.76 & 0.31 & 5.88 & 0.31 & 0.20 & 0.25 \\
\hline 8 & 6.98 & 0.30 & 4.99 & 0.27 & 0.90 & 0.19 \\
\hline 9 & 6.84 & 0.04 & 4.64 & 0.19 & 0.60 & 0.06 \\
\hline 10 & 7.24 & 0.15 & 6.25 & 0.22 & 0.70 & 0.13 \\
\hline 11 & 6.98 & 0.20 & 5.77 & 0.20 & 0.80 & 0.10 \\
\hline 12 & 6.74 & 0.46 & 5.31 & 0.41 & 1.20 & 0.37 \\
\hline 13 & 6.92 & 0.12 & 5.32 & 0.19 & 0.70 & 0.06 \\
\hline 14 & 7.65 & 0.24 & 5.21 & 0.30 & 0.20 & 0.15 \\
\hline 15 & 6.84 & 0.11 & 5.26 & 0.22 & 1.20 & 0.09 \\
\hline 16 & 6.52 & 0.30 & 5.29 & 0.37 & 1.40 & 0.32 \\
\hline 17 & 7.42 & 0.30 & 5.23 & 0.30 & 0.30 & 0.21 \\
\hline 18 & 6.72 & 0.45 & 4.49 & 0.42 & 0.70 & 0.38 \\
\hline 19 & 7.12 & 0.20 & 5.49 & 0.25 & 0.80 & 0.17 \\
\hline 20 & 7.76 & 0.27 & 6.26 & 0.29 & 1.00 & 0.22 \\
\hline 21 & 7.57 & 0.27 & 6.30 & 0.29 & 1.40 & 0.22 \\
\hline 22 & 8.16 & 0.84 & 6.01 & 0.69 & 0.20 & 0.74 \\
\hline 23 & 6.42 & 0.42 & 5.91 & 0.47 & 1.90 & 0.44 \\
\hline
\end{tabular}

Table 31

Observed Properties of Star Clusters in NGC 3690W

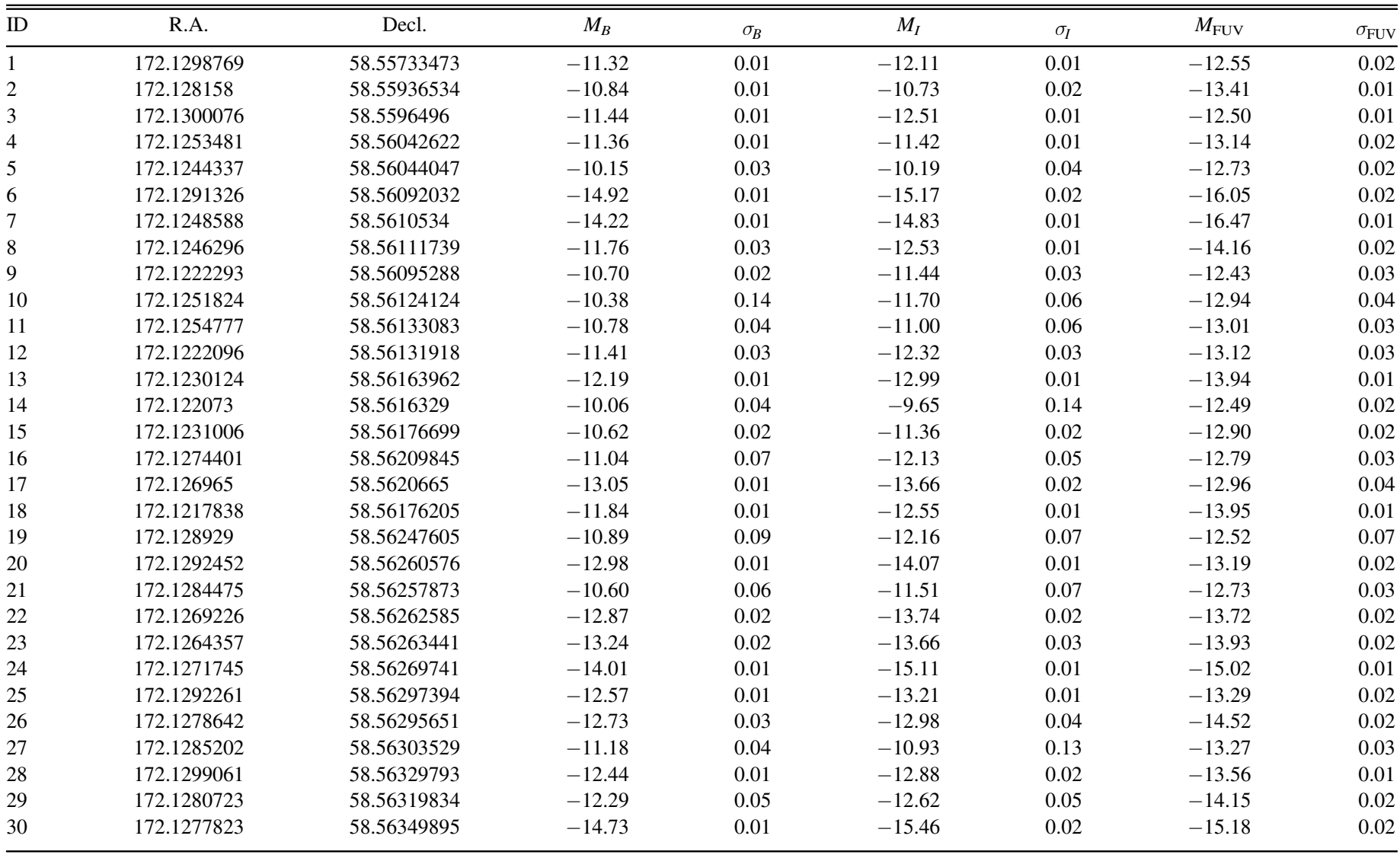


Table 32

Derived Properties of Star Clusters in NGC 3690W

\begin{tabular}{|c|c|c|c|c|c|c|}
\hline ID & $\log ($ Age $)$ & $\sigma_{\mathrm{Age}}$ & $\log \left(M / M_{\odot}\right)$ & $\sigma_{M}$ & $A_{V}$ & $\sigma_{A_{V}}$ \\
\hline 1 & 6.78 & 0.02 & 5.27 & 0.18 & 1.00 & 0.05 \\
\hline 2 & 6.42 & 0.16 & 5.03 & 0.19 & 0.70 & 0.06 \\
\hline 3 & 7.63 & 0.34 & 6.00 & 0.30 & 0.70 & 0.23 \\
\hline 4 & 6.66 & 0.10 & 5.07 & 0.17 & 0.70 & 1.12 \\
\hline 5 & 5.10 & 0.40 & 5.03 & 0.18 & 0.90 & 0.04 \\
\hline 6 & 6.66 & 8.74 & 6.70 & 0.18 & 1.10 & 0.03 \\
\hline 7 & 6.84 & 0.01 & 6.26 & 0.17 & 0.50 & 0.30 \\
\hline 8 & 6.88 & 0.01 & 5.35 & 0.17 & 0.50 & 0.17 \\
\hline 9 & 6.84 & 0.01 & 4.95 & 0.18 & 0.70 & 0.04 \\
\hline 10 & 5.10 & 0.01 & 6.71 & 0.23 & 3.90 & 0.02 \\
\hline 11 & 5.10 & 0.67 & 5.39 & 0.29 & 1.10 & 0.22 \\
\hline 12 & 6.88 & 0.04 & 5.32 & 0.18 & 0.70 & 0.05 \\
\hline 13 & 6.86 & 0.10 & 5.59 & 0.17 & 0.70 & 1.12 \\
\hline 14 & 6.52 & 0.02 & 4.40 & 0.19 & 0.50 & 0.06 \\
\hline 15 & 6.88 & 0.01 & 4.90 & 0.17 & 0.50 & 0.20 \\
\hline 16 & 7.42 & 0.20 & 5.65 & 0.21 & 0.50 & 0.09 \\
\hline 17 & 8.31 & 0.35 & 6.95 & 0.35 & 0.50 & 0.30 \\
\hline 18 & 6.86 & 0.01 & 5.34 & 0.17 & 0.50 & 0.99 \\
\hline 19 & 5.10 & 0.19 & 6.92 & 0.24 & 3.90 & 0.12 \\
\hline 20 & 8.01 & 0.48 & 6.89 & 0.41 & 0.80 & 0.37 \\
\hline 21 & 6.90 & 0.10 & 4.94 & 0.19 & 0.50 & 0.42 \\
\hline 22 & 6.74 & 0.58 & 6.02 & 0.44 & 1.30 & 0.41 \\
\hline 23 & 6.66 & 6.61 & 6.19 & 0.18 & 1.40 & 0.04 \\
\hline 24 & 7.65 & 0.29 & 7.05 & 0.25 & 0.70 & 0.18 \\
\hline 25 & 6.62 & 0.06 & 5.92 & 0.23 & 1.50 & 0.14 \\
\hline 26 & 6.38 & 0.49 & 6.18 & 0.36 & 1.30 & 0.30 \\
\hline 27 & 6.66 & 0.08 & 4.89 & 0.22 & 0.50 & 0.12 \\
\hline 28 & 6.64 & 0.06 & 5.73 & 0.23 & 1.20 & 0.15 \\
\hline 29 & 6.72 & 0.43 & 5.40 & 0.35 & 0.60 & 0.30 \\
\hline 30 & 6.68 & 0.03 & 6.89 & 0.18 & 1.60 & 0.05 \\
\hline
\end{tabular}

Table 33

Observed Properties of Star Clusters in NGC 5257E

\begin{tabular}{|c|c|c|c|c|c|c|c|c|}
\hline ID & R.A. & Decl. & $M_{B}$ & $\sigma_{B}$ & $M_{I}$ & $\sigma_{I}$ & $M_{\mathrm{FUV}}$ & $\sigma_{\mathrm{FUV}}$ \\
\hline 1 & 204.988389 & 0.82941763 & -15.90 & 0.01 & -16.62 & 0.02 & -15.81 & 0.01 \\
\hline 2 & 204.9886135 & 0.828844213 & -13.70 & 0.01 & -14.98 & 0.01 & -12.25 & 0.07 \\
\hline 3 & 204.9893355 & 0.829802618 & -12.13 & 0.01 & -12.41 & 0.04 & -12.86 & 0.04 \\
\hline 4 & 204.99099 & 0.832602833 & -12.33 & 0.01 & -12.80 & 0.02 & -13.84 & 0.02 \\
\hline 5 & 204.9913336 & 0.832908072 & -12.27 & 0.05 & -12.88 & 0.03 & -13.58 & 0.04 \\
\hline 6 & 204.9893535 & 0.828699614 & -12.67 & 0.02 & -13.22 & 0.03 & -13.46 & 0.02 \\
\hline 7 & 204.9910317 & 0.83185232 & -13.36 & 0.01 & -14.25 & 0.02 & -13.32 & 0.03 \\
\hline 8 & 204.9915112 & 0.832786319 & -12.80 & 0.06 & -13.26 & 0.05 & -13.77 & 0.05 \\
\hline 9 & 204.992339 & 0.834417957 & -11.11 & 0.10 & -11.94 & 0.07 & -11.23 & 0.18 \\
\hline 10 & 204.9921984 & 0.834047212 & -11.78 & 0.04 & -12.36 & 0.05 & -12.99 & 0.04 \\
\hline 11 & 204.9928594 & 0.834311205 & -13.27 & 0.01 & -13.81 & 0.01 & -14.38 & 0.01 \\
\hline 12 & 204.9927539 & 0.833729231 & -12.57 & 0.02 & -13.18 & 0.02 & -13.46 & 0.02 \\
\hline 13 & 204.9917487 & 0.831103146 & -14.21 & 0.02 & -15.08 & 0.02 & -13.94 & 0.01 \\
\hline 14 & 204.9941781 & 0.833654938 & -13.58 & 0.01 & -15.28 & 0.01 & -12.41 & 0.06 \\
\hline 15 & 204.9935202 & 0.831994291 & -15.04 & 0.01 & -15.07 & 0.01 & -17.12 & 0.01 \\
\hline 16 & 204.993914 & 0.832897563 & -12.26 & 0.04 & -12.65 & 0.05 & -14.00 & 0.01 \\
\hline
\end{tabular}

\section{UGC 04881}

UGC 04881 is an early-stage merger containing two nuclei separated by $\sim 11^{\prime \prime}(9 \mathrm{kpc})$. Spiral dust lanes and strings of star clusters surround the NE nucleus. In the SW nucleus, a linear distribution of star clusters and a prominent dust lane are seen (Figure 25). The maximum $A_{V}$ adopted for this galaxy is $1.9 \mathrm{mag}$ of visual extinction (see Figure 26; González-Martín et al. 2009).
The observed and derived properties for the star clusters identified in this galaxy are given in Tables 21 and 22, respectively.

\section{IC 2545}

IC 2545 is a late-stage merger being viewed face on. Dust lanes and strings of star clusters extend from two unresolved 
Table 34

Derived Properties of Star Clusters in NGC 5257E

\begin{tabular}{|c|c|c|c|c|c|c|}
\hline ID & $\log ($ Age $)$ & $\sigma_{\mathrm{Age}}$ & $\log \left(M / M_{\odot}\right)$ & $\sigma_{M}$ & $A_{V}$ & $\sigma_{A V}$ \\
\hline 1 & 8.31 & 0.01 & 8.04 & 0.17 & 0.40 & 0.23 \\
\hline 2 & 8.56 & 0.03 & 7.46 & 0.18 & 0.60 & 0.07 \\
\hline 3 & 6.66 & 0.39 & 5.75 & 0.17 & 1.40 & 0.04 \\
\hline 4 & 6.72 & 0.67 & 5.58 & 0.39 & 0.90 & 0.34 \\
\hline 5 & 6.72 & 0.58 & 5.61 & 0.36 & 1.00 & 0.30 \\
\hline 6 & 6.66 & 0.03 & 5.96 & 0.19 & 1.40 & 0.07 \\
\hline 7 & 8.31 & 0.49 & 7.02 & 0.48 & 0.40 & 0.46 \\
\hline 8 & 6.66 & 0.40 & 5.96 & 0.29 & 1.30 & 0.22 \\
\hline 9 & 6.66 & 0.81 & 5.60 & 0.66 & 1.90 & 0.70 \\
\hline 10 & 6.00 & 0.44 & 6.06 & 0.37 & 1.80 & 0.32 \\
\hline 11 & 6.30 & 0.09 & 6.68 & 0.22 & 1.80 & 0.13 \\
\hline 12 & 6.52 & 0.39 & 6.04 & 0.31 & 1.70 & 0.24 \\
\hline 13 & 8.36 & 0.02 & 7.45 & 0.18 & 0.50 & 0.06 \\
\hline 14 & 8.01 & 0.05 & 7.66 & 0.18 & 1.80 & 0.04 \\
\hline 15 & 6.56 & 0.05 & 6.49 & 0.21 & 0.70 & 0.11 \\
\hline 16 & 6.72 & 0.43 & 5.45 & 0.36 & 0.70 & 0.31 \\
\hline
\end{tabular}

Table 35

Observed Properties of Star Clusters in NGC 5257W

\begin{tabular}{|c|c|c|c|c|c|c|c|c|}
\hline ID & R.A. & Decl. & $M_{B}$ & $\sigma_{B}$ & $M_{I}$ & $\sigma_{I}$ & $M_{\mathrm{FUV}}$ & $\sigma_{\mathrm{FUV}}$ \\
\hline 1 & 204.9677325 & 0.839888315 & -12.60 & 0.04 & -13.26 & 0.04 & -13.43 & 0.07 \\
\hline 2 & 204.9676008 & 0.839405141 & -14.32 & 0.02 & -14.40 & 0.02 & -16.43 & 0.01 \\
\hline 3 & 204.9676117 & 0.839232784 & -13.47 & 0.04 & -13.90 & 0.05 & -14.56 & 0.02 \\
\hline 4 & 204.9679115 & 0.838666338 & -12.25 & 0.06 & -13.29 & 0.02 & -14.24 & 0.06 \\
\hline 5 & 204.9680284 & 0.838781875 & -13.85 & 0.02 & -14.10 & 0.03 & -15.44 & 0.01 \\
\hline 6 & 204.9679985 & 0.838669059 & -13.07 & 0.06 & -13.32 & 0.06 & -15.08 & 0.04 \\
\hline 7 & 204.969473 & 0.840889786 & -13.17 & 0.01 & -13.46 & 0.01 & -14.21 & 0.01 \\
\hline 8 & 204.9690871 & 0.839750205 & -12.72 & 0.05 & -13.41 & 0.07 & -13.63 & 0.02 \\
\hline 9 & 204.96912 & 0.837489752 & -13.63 & 0.05 & -14.12 & 0.08 & -14.60 & 0.06 \\
\hline 10 & 204.9692242 & 0.837503427 & -15.05 & 0.02 & -15.74 & 0.02 & -16.38 & 0.01 \\
\hline 11 & 204.970253 & 0.837864699 & -12.01 & 0.02 & -12.17 & 0.04 & -14.13 & 0.01 \\
\hline 12 & 204.9727531 & 0.841813782 & -11.72 & 0.06 & -11.67 & 0.09 & -13.63 & 0.04 \\
\hline 13 & 204.9703117 & 0.836690288 & -13.97 & 0.02 & -14.12 & 0.03 & -15.22 & 0.03 \\
\hline 14 & 204.9728795 & 0.841450503 & -13.65 & 0.02 & -13.86 & 0.03 & -16.06 & 0.01 \\
\hline 15 & 204.9730222 & 0.841315721 & -12.87 & 0.04 & -13.54 & 0.03 & -14.71 & 0.02 \\
\hline 16 & 204.9731456 & 0.841116419 & -14.47 & 0.01 & -14.61 & 0.02 & -16.80 & 0.01 \\
\hline 17 & 204.9735238 & 0.840747 & -12.24 & 0.06 & -12.70 & 0.07 & -13.80 & 0.08 \\
\hline 18 & 204.9737106 & 0.840991159 & -12.21 & 0.04 & -12.65 & 0.07 & -14.41 & 0.02 \\
\hline 19 & 204.9738917 & 0.840626292 & -12.65 & 0.04 & -13.15 & 0.03 & -14.44 & 0.02 \\
\hline 20 & 204.9736297 & 0.838529841 & -13.22 & 0.03 & -14.02 & 0.02 & -14.44 & 0.02 \\
\hline 21 & 204.974667 & 0.839754239 & -12.11 & 0.03 & -11.73 & 0.09 & -14.16 & 0.02 \\
\hline 22 & 204.9746698 & 0.838945129 & -12.42 & 0.02 & -12.28 & 0.05 & -14.85 & 0.01 \\
\hline
\end{tabular}

nuclei separated by $\sim 0$." $8(0.54 \mathrm{kpc})$ in the center of the galaxy. Multiple star clusters are also visible throughout the tidal tails (Figure 27). The maximum $A_{V}$ adopted for this galaxy is $4.0 \mathrm{mag}$ of visual extinction (see Figure 28; van den Broek et al. 1991). The observed and derived properties for the star clusters identified in this galaxy are given in Tables 23 and 24, respectively.

\section{NGC 3256}

NGC 3256 is a late-stage merger containing a large number of star clusters along the inner $\left(\sim 20^{\prime \prime}\right.$, or $\left.4 \mathrm{kpc}\right)$ spiral structure of the nuclear region. The spiral dust lanes extending from the nucleus give this galaxy pockets of high and low extinction (Figure 29). The maximum $A_{V}$ adopted for this galaxy is $3.3 \mathrm{mag}$ of visual extinction (see Figure 30; Rich et al. 2012). The observed and derived properties for the star clusters identified in this galaxy are given in Tables 25 and 26 , respectively.

\section{Arp 148}

Arp 148 is an early-stage merger and the only example of a ring galaxy in the sample. This $\sim 23^{\prime \prime}(16 \mathrm{kpc})$ diameter galaxy is comprised of clumps of star clusters along its perimeter and throughout much of its interior (Figure 31). The maximum $A_{V}$ adopted for this galaxy is $2.1 \mathrm{mag}$ of visual extinction (see 
Table 36

Derived Properties of Star Clusters in NGC 5257W

\begin{tabular}{|c|c|c|c|c|c|c|}
\hline ID & $\log ($ Age $)$ & $\sigma_{\text {Age }}$ & $\log \left(M / M_{\odot}\right)$ & $\sigma_{M}$ & $A_{V}$ & $\sigma_{A_{V}}$ \\
\hline 1 & 6.56 & 0.64 & 6.19 & 0.59 & 1.80 & 0.60 \\
\hline 2 & 6.48 & 0.09 & 6.57 & 0.22 & 1.10 & 0.13 \\
\hline 3 & 6.48 & 0.04 & 6.60 & 0.20 & 1.80 & 0.09 \\
\hline 4 & 6.24 & 0.02 & 6.35 & 0.20 & 1.80 & 0.07 \\
\hline 5 & 6.54 & 0.03 & 6.42 & 0.19 & 1.30 & 0.07 \\
\hline 6 & 6.72 & 0.34 & 5.84 & 0.32 & 0.70 & 0.26 \\
\hline 7 & 6.54 & 0.01 & 6.30 & 0.18 & 1.60 & 0.05 \\
\hline 8 & 6.64 & 0.59 & 6.25 & 0.54 & 1.70 & 0.53 \\
\hline 9 & 6.54 & 0.29 & 6.54 & 0.35 & 1.70 & 0.29 \\
\hline 10 & 6.74 & 0.32 & 6.83 & 0.29 & 1.10 & 0.23 \\
\hline 11 & 6.58 & 0.30 & 5.50 & 0.29 & 0.90 & 0.22 \\
\hline 12 & 6.54 & 0.03 & 5.40 & 0.21 & 1.00 & 0.09 \\
\hline 13 & 6.52 & 0.01 & 6.57 & 0.18 & 1.50 & 0.03 \\
\hline 14 & 6.74 & 0.01 & 5.90 & 0.17 & 0.40 & 0.02 \\
\hline 15 & 7.30 & 0.19 & 6.19 & 0.21 & 0.50 & 0.10 \\
\hline 16 & 6.72 & 0.01 & 6.29 & 0.18 & 0.50 & 0.05 \\
\hline 17 & 6.58 & 0.24 & 5.81 & 0.31 & 1.30 & 0.24 \\
\hline 18 & 6.76 & 0.16 & 5.37 & 0.19 & 0.50 & 0.07 \\
\hline 19 & 6.74 & 0.17 & 5.71 & 0.19 & 0.80 & 0.07 \\
\hline 20 & 7.63 & 0.27 & 6.60 & 0.26 & 0.60 & 0.19 \\
\hline 21 & 6.52 & 0.01 & 5.51 & 0.18 & 0.90 & 0.05 \\
\hline 22 & 6.52 & 0.05 & 5.53 & 0.20 & 0.70 & 0.10 \\
\hline
\end{tabular}

Table 37

Observed Properties of Star Clusters in NGC 5331S

\begin{tabular}{cccccccc}
\hline \hline ID & R.A. & Decl. & $M_{B}$ & $\sigma_{B}$ & $M_{I}$ & $\sigma_{I}$ & $M_{\text {FUV }}$ \\
\hline 1 & 208.0703109 & 2.098268967 & -9.51 & 0.10 & -10.42 & 0.10 & -9.83 \\
2 & 208.0667504 & 2.102795958 & -12.63 & 0.03 & -13.47 & 0.03 & -8.96 \\
3 & 208.0676478 & 2.100879121 & -15.03 & 0.01 & -15.70 & 0.01 & -10.76 \\
4 & 208.0681184 & 2.100981506 & -12.59 & 0.02 & -13.40 & 0.03 & -9.66 \\
5 & 208.0672543 & 2.099466214 & -9.90 & 0.07 & -12.06 & 0.03 & -9.13 \\
6 & 208.0680338 & 2.099718103 & -9.64 & 0.10 & -11.49 & 0.10 & -9.19 \\
7 & 208.068045 & 2.100690283 & -13.34 & 0.03 & -14.10 & 0.02 & -9.69 \\
\hline
\end{tabular}

Table 38

Derived Properties of Star Clusters in NGC 5331S

\begin{tabular}{|c|c|c|c|c|c|c|}
\hline ID & Log(Age) & $\sigma_{\text {Age }}$ & $\log \left(M / M_{\odot}\right)$ & $\sigma_{M}$ & $A_{V}$ & $\sigma_{A_{V}}$ \\
\hline 1 & 6.50 & 0.38 & 5.53 & 0.20 & 2.30 & 0.37 \\
\hline 2 & 8.76 & 0.58 & 8.08 & 0.17 & 1.80 & 0.55 \\
\hline 3 & 8.86 & 0.44 & 9.13 & 0.17 & 1.80 & 0.31 \\
\hline 4 & 8.61 & 0.43 & 7.93 & 0.17 & 1.80 & 0.28 \\
\hline 5 & 6.80 & 0.35 & 5.85 & 0.19 & 2.50 & 0.92 \\
\hline 6 & 6.82 & 0.51 & 5.46 & 0.20 & 1.90 & 0.70 \\
\hline 7 & 7.91 & 0.13 & 8.70 & 0.18 & 3.60 & 0.05 \\
\hline
\end{tabular}

Figure 32; Joy \& Harvey 1987). The observed and derived properties for the star clusters identified in this galaxy are given in Tables 27 and 28, respectively.

\section{NGC $3690 E$}

NGC 3690 is a mid-stage merger. NGC 3690E contains a multitude of star clusters and dust lanes from the southeast tip of the galaxy to the northwest (Figure 33). The maximum $A_{V}$ adopted for this galaxy is 3.4 mag of visual extinction (see Figure 34; García-Marín et al. 2006). The observed and derived properties for the star clusters identified in this galaxy are given in Tables 29 and 30, respectively.

\section{NGC 3690W}

NGC $3690 \mathrm{~W}$ has the brightest cluster complexes in the merging system located $\sim 6$ !"8 $(1.6 \mathrm{kpc})$ from the resolved 
Table 39

Observed Properties of Star Clusters in NGC 5331N

\begin{tabular}{|c|c|c|c|c|c|c|c|c|}
\hline ID & R.A. & Decl. & $M_{B}$ & $\sigma_{B}$ & $M_{I}$ & $\sigma_{I}$ & $M_{\mathrm{FUV}}$ & $\sigma_{\mathrm{FUV}}$ \\
\hline 1 & 208.0660418 & 2.10796331 & -9.62618 & 0.07 & -10.20 & 0.10 & -11.87 & 0.10 \\
\hline 2 & 208.0660027 & 2.107205416 & -9.75618 & 0.07 & -10.89 & 0.07 & -10.83 & 0.07 \\
\hline 3 & 208.0667669 & 2.107753228 & -9.37618 & 0.09 & -10.89 & 0.08 & -10.12 & 0.11 \\
\hline 5 & 208.0682536 & 2.109496656 & -11.5962 & 0.02 & -11.98 & 0.05 & -12.80 & 0.04 \\
\hline 6 & 208.0667871 & 2.107126587 & -11.0962 & 0.03 & -11.62 & 0.05 & -11.60 & 0.14 \\
\hline 7 & 208.0674622 & 2.107317376 & -12.2362 & 0.03 & -13.18 & 0.04 & -13.14 & 0.03 \\
\hline 10 & 208.0683038 & 2.10672568 & -11.4162 & 0.03 & -11.50 & 0.08 & -12.26 & 0.07 \\
\hline 11 & 208.0686796 & 2.107921587 & -13.8362 & 0.01 & -14.44 & 0.01 & -13.80 & 0.02 \\
\hline 12 & 208.0666683 & 2.102976709 & -12.5562 & 0.02 & -13.41 & 0.02 & -11.52 & 0.01 \\
\hline 13 & 208.0698361 & 2.108671969 & -10.3762 & 0.09 & -10.51 & 0.14 & -11.62 & 0.12 \\
\hline 14 & 208.0685396 & 2.107683102 & -11.6562 & 0.07 & -13.34 & 0.02 & -11.38 & 0.16 \\
\hline 15 & 208.0699658 & 2.111094004 & -10.4662 & 0.03 & -11.17 & 0.03 & -9.81 & 0.13 \\
\hline
\end{tabular}

Table 40

Derived Properties of Star Clusters in NGC 5331N

\begin{tabular}{|c|c|c|c|c|c|c|}
\hline ID & $\log ($ Age $)$ & $\sigma_{\text {Age }}$ & $\log \left(M / M_{\odot}\right)$ & $\sigma_{M}$ & $A_{V}$ & $\sigma_{A_{V}}$ \\
\hline 1 & 6.86 & 0.33 & 4.64 & 0.25 & 0.30 & 0.15 \\
\hline 2 & 7.54 & 0.34 & 5.65 & 0.30 & 0.80 & 0.23 \\
\hline 3 & 7.42 & 0.24 & 5.65 & 0.27 & 1.20 & 0.17 \\
\hline 5 & 6.66 & 0.11 & 5.67 & 0.27 & 1.10 & 0.20 \\
\hline 6 & 8.31 & 0.01 & 6.20 & 0.18 & 0.01 & 0.04 \\
\hline 7 & 6.78 & 0.44 & 6.09 & 0.36 & 1.30 & 0.30 \\
\hline 10 & 6.66 & 0.04 & 5.70 & 0.19 & 1.30 & 0.07 \\
\hline 11 & 6.68 & 0.30 & 6.94 & 0.17 & 1.80 & 0.08 \\
\hline 12 & 8.61 & 0.15 & 7.08 & 0.18 & 0.10 & 0.05 \\
\hline 13 & 6.66 & 0.35 & 5.13 & 0.28 & 1.00 & 0.19 \\
\hline 14 & 7.65 & 0.11 & 6.88 & 0.22 & 1.60 & 0.11 \\
\hline 15 & 8.56 & 0.02 & 6.14 & 0.17 & 0.01 & 0.13 \\
\hline
\end{tabular}

nucleus. Cluster-rich spiral arms extend north and westward from the nuclear region out to a maximum projected distance of $\sim 58^{\prime \prime}$ (14 kpc; Figure 33). The maximum $A_{V}$ adopted for this galaxy is $3.9 \mathrm{mag}$ of visual extinction (see Figure 35; García-Marín et al. 2006). The observed and derived properties for the star clusters identified in this galaxy are given in Tables 31 and 32, respectively.

\section{NGC 5257E}

NGC $5257 / 8$ is an early-stage merger system with the E and $\mathrm{W}$ nuclei separated by $\sim 80^{\prime \prime}(40 \mathrm{kpc})$. Star clusters and dust lanes make up the prominent spiral arms seen in the eastern galaxy (Figure 36). The maximum $A_{V}$ adopted for this galaxy is $2.6 \mathrm{mag}$ of visual extinction (see Figure 37; Smith et al. 2014). The observed and derived properties for the star clusters identified in this galaxy are given in Tables 33 and 34 , respectively.

\section{NGC $5257 \mathrm{~W}$}

NGC $5257 \mathrm{~W}$ contains a prominent group of bright clusters located $\sim 10^{\prime \prime}(5 \mathrm{kpc})$ south from the nucleus in a $\sim 17^{\prime \prime}$ $(4.2 \mathrm{kpc})$ long spiral arm (Figure 38$)$. The maximum $A_{V}$ adopted for this galaxy is 1.8 mag of visual extinction (see Figure 39; Smith et al. 2014). The observed and derived properties for the star clusters identified in this galaxy are given in Tables 35 and 36, respectively.

\section{$N G C 5331 S$}

NGC 5331 is a mid-stage merging system. NGC 5331S and $\mathrm{N}$ have a projected nuclear separation of $\sim 27^{\prime \prime}(19 \mathrm{kpc})$. Large dust lanes are visible along the near edge of the galaxy, and only a small number of star clusters are visible (Figure 40). The maximum $A_{V}$ adopted for this galaxy is $3.6 \mathrm{mag}$ of visual extinction (see Figure 41; Lutz 1992). The observed and derived properties for the star clusters 
Table 41

Observed Properties of Star Clusters in UGC 09618NED02

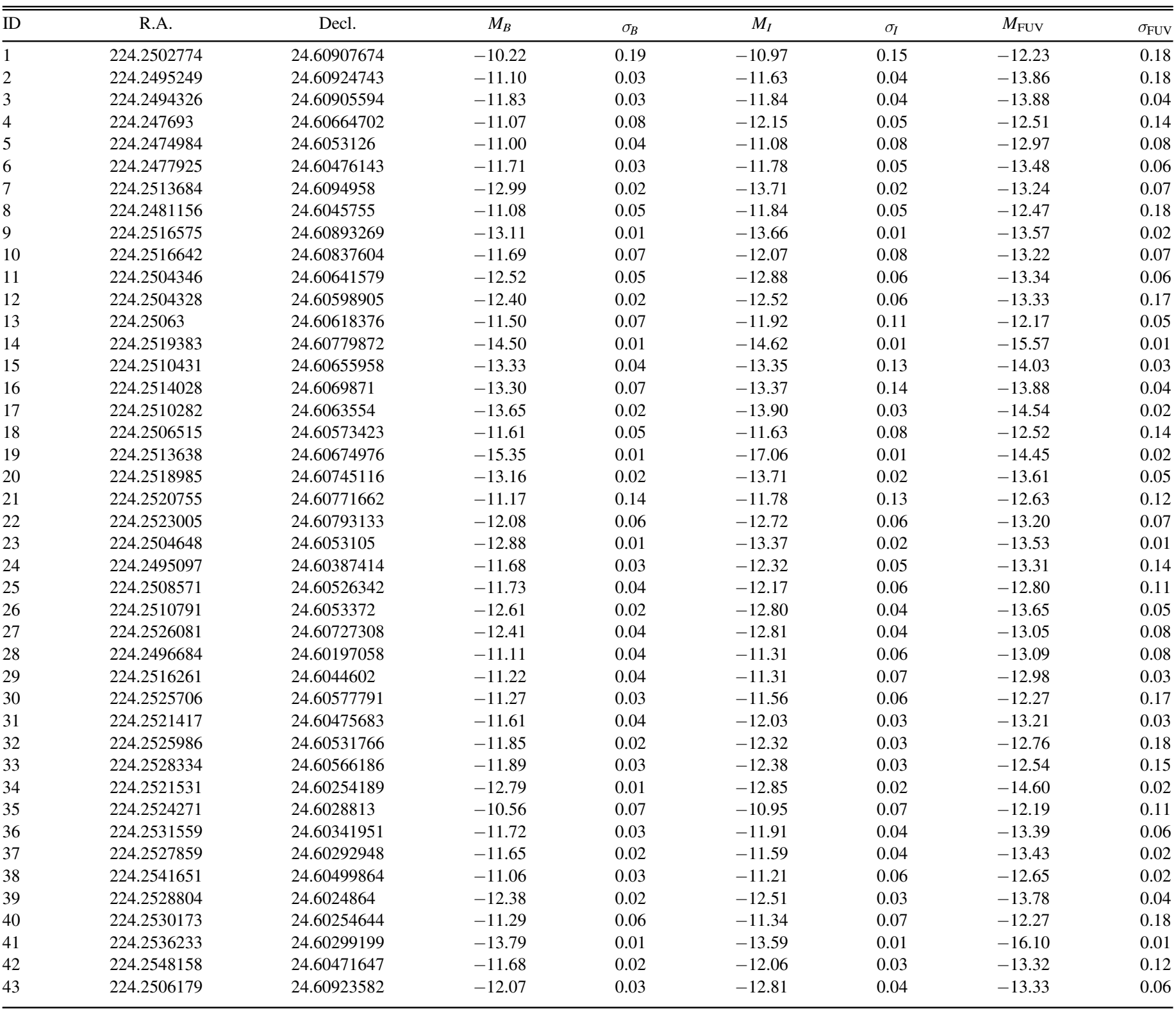

identified in this galaxy are given in Tables 37 and 38, respectively.

\section{NGC $5331 N$}

NGC 5331N has a nucleus and two distinct spiral arms, with a small number of star clusters visible throughout the galaxy (Figure 40). The maximum $A_{V}$ adopted for this galaxy is $1.8 \mathrm{mag}$ of visual extinction (see Figure 42; Lutz 1992). The observed and derived properties for the star clusters identified in this galaxy are given in Tables 39 and 40, respectively.

\section{UGC 09618NED02}

Armus et al. (2009) discusses the detailed morphology of this galaxy at length. UGC 09618NED02 is an early-stage merger with the two nuclei separated by $\sim 40^{\prime \prime}(30 \mathrm{kpc})$. Multiple star clusters are visible along the spiral arms in the face-on galaxy (VV430A; Figure 43). The maximum $A_{V}$ adopted for this galaxy is $2.4 \mathrm{mag}$ of visual extinction (see Figure 44; Leech et al. 1989). The observed and derived properties for the star clusters identified in this galaxy are given in Tables 41 and 42, respectively.

\section{IC $4687 N$}

IC 4687 is an early-stage merging system. The two primary galaxies (IC 4686 and IC 4687) have a nuclear separation of $\sim 84^{\prime \prime}$ (31 kpc). The northern galaxy contains several bright clusters in a nuclear arm stretching north and westward (Figure 45). The maximum $A_{V}$ adopted for this galaxy is 2.8 mag of visual extinction (see Figure 46; Rich et al. 2012). 
Table 42

Derived Properties of Star Clusters in UGC 09618NED02

\begin{tabular}{|c|c|c|c|c|c|c|}
\hline ID & $\log ($ Age $)$ & $\sigma_{\mathrm{Age}}$ & $\log \left(M / M_{\odot}\right)$ & $\sigma_{M}$ & $A_{V}$ & $\sigma_{A_{V}}$ \\
\hline 1 & 7.57 & 0.39 & 5.17 & 0.41 & 0.10 & 0.32 \\
\hline 2 & 6.88 & 0.05 & 4.82 & 0.16 & 0.01 & 0.45 \\
\hline 3 & 6.66 & 0.09 & 5.15 & 0.25 & 0.50 & 0.18 \\
\hline 4 & 7.63 & 0.28 & 5.70 & 0.28 & 0.40 & 0.21 \\
\hline 5 & 6.64 & 0.20 & 4.84 & 0.34 & 0.60 & 0.29 \\
\hline 6 & 6.66 & 0.03 & 5.21 & 0.19 & 0.70 & 0.09 \\
\hline 7 & 8.41 & 0.03 & 6.73 & 0.17 & 0.01 & 0.05 \\
\hline 8 & 6.76 & 0.52 & 5.10 & 0.38 & 0.90 & 0.34 \\
\hline 9 & 6.66 & 0.02 & 6.25 & 0.16 & 1.60 & 0.04 \\
\hline 10 & 6.38 & 0.65 & 5.87 & 0.53 & 1.50 & 0.52 \\
\hline 11 & 6.66 & 0.57 & 5.85 & 0.49 & 1.30 & 0.47 \\
\hline 12 & 6.66 & 0.53 & 5.75 & 0.16 & 1.20 & 0.03 \\
\hline 13 & 6.66 & 0.75 & 5.55 & 0.63 & 1.50 & 0.66 \\
\hline 14 & 6.66 & 0.95 & 6.54 & 0.16 & 1.10 & 0.03 \\
\hline 15 & 6.66 & 0.57 & 6.18 & 0.48 & 1.30 & 0.46 \\
\hline 16 & 6.66 & 0.77 & 6.22 & 0.61 & 1.40 & 0.63 \\
\hline 17 & 6.66 & 0.67 & 6.30 & 0.16 & 1.30 & 0.04 \\
\hline 18 & 6.66 & 0.16 & 5.43 & 0.18 & 1.20 & 0.05 \\
\hline 19 & 7.86 & 0.04 & 8.29 & 0.17 & 1.80 & 0.05 \\
\hline 20 & 8.36 & 0.03 & 6.76 & 0.16 & 0.01 & 0.03 \\
\hline 21 & 6.74 & 0.75 & 5.13 & 0.53 & 0.90 & 0.51 \\
\hline 22 & 5.10 & 0.97 & 6.34 & 0.66 & 1.90 & 0.70 \\
\hline 23 & 6.66 & 0.03 & 6.10 & 0.16 & 1.50 & 0.02 \\
\hline 24 & 6.76 & 0.47 & 5.29 & 0.37 & 0.80 & 0.33 \\
\hline 25 & 6.66 & 0.26 & 5.48 & 0.35 & 1.20 & 0.31 \\
\hline 26 & 6.66 & 0.51 & 5.83 & 0.17 & 1.20 & 0.05 \\
\hline 27 & 6.66 & 0.75 & 5.91 & 0.61 & 1.50 & 0.63 \\
\hline 28 & 6.36 & 0.46 & 5.45 & 0.36 & 1.20 & 0.31 \\
\hline 29 & 6.66 & 0.09 & 5.01 & 0.24 & 0.70 & 0.17 \\
\hline 30 & 6.66 & 0.43 & 5.30 & 0.17 & 1.20 & 0.04 \\
\hline 31 & 6.72 & 0.64 & 5.24 & 0.47 & 0.80 & 0.45 \\
\hline 32 & 6.66 & 0.01 & 5.58 & 0.16 & 1.30 & 0.02 \\
\hline 33 & 6.66 & 0.03 & 5.71 & 0.16 & 1.50 & 0.04 \\
\hline 34 & 6.66 & 0.01 & 5.64 & 0.16 & 0.70 & 0.03 \\
\hline 35 & 6.00 & 0.65 & 5.42 & 0.50 & 1.50 & 0.48 \\
\hline 36 & 6.66 & 0.25 & 5.27 & 0.25 & 0.80 & 0.18 \\
\hline 37 & 6.66 & 0.86 & 5.19 & 0.16 & 0.70 & 0.04 \\
\hline 38 & 6.66 & 0.04 & 5.00 & 0.20 & 0.80 & 0.11 \\
\hline 39 & 6.66 & 0.07 & 5.58 & 0.17 & 0.90 & 0.05 \\
\hline 40 & 6.66 & 0.35 & 5.31 & 0.34 & 1.20 & 0.28 \\
\hline 41 & 6.66 & 0.36 & 5.83 & 0.16 & 0.30 & 0.06 \\
\hline 42 & 5.10 & 0.67 & 5.96 & 0.38 & 1.50 & 0.34 \\
\hline 43 & 6.78 & 0.55 & 5.57 & 0.43 & 1.00 & 0.40 \\
\hline
\end{tabular}

The observed and derived properties for the star clusters identified in this galaxy are given in Tables 43 and 44, respectively.

\section{IC $4687 S$}

The southern galaxy contains several bright clusters in the nuclear region as well as a series of dust lanes. Their is little evidence of extended tidal structures which contain star clusters (Figure 47). The maximum $A_{V}$ adopted for this galaxy is 3.7 mag of visual extinction (see Figure 48; Rich et al. 2012). The observed and derived properties for the star clusters identified in this galaxy are given in Tables 45 and 46, respectively.

\section{NGC 6786}

NGC 6786 is an early-stage merger consisting of a pair of face-on galaxies with a nuclear separation of $\sim 72^{\prime \prime}$ ( $37 \mathrm{kpc}$ ). Faint star clusters are seen along the inner spiral structure. The brightest clusters sit at roughly $5^{\prime \prime}(3 \mathrm{kpc})$ from the southwest nucleus in the large arm/tail extending between the two galaxies (Figure 49). The maximum $A_{V}$ adopted for this galaxy is $2.0 \mathrm{mag}$ of visual extinction (see Figure 50; Martin et al. 1991). The observed and derived properties for the star clusters 
Table 43

Observed Properties of Star Clusters in IC 4687N

\begin{tabular}{|c|c|c|c|c|c|c|c|c|}
\hline ID & R.A. & Decl. & $M_{B}$ & $\sigma_{B}$ & $M_{I}$ & $\sigma_{I}$ & $M_{\mathrm{FUV}}$ & $\sigma_{\mathrm{FUV}}$ \\
\hline 1 & 273.4245591 & -57.72132281 & -8.50 & 0.12 & -9.60 & 0.08 & -11.68 & 0.07 \\
\hline 2 & 273.4139193 & -57.72470603 & -12.61 & 0.02 & -14.37 & 0.02 & -11.88 & 0.10 \\
\hline 3 & 273.4144883 & -57.72459737 & -14.21 & 0.03 & -14.55 & 0.03 & -15.57 & 0.02 \\
\hline 4 & 273.4149949 & -57.72446495 & -13.59 & 0.03 & -14.94 & 0.03 & -14.02 & 0.07 \\
\hline 5 & 273.4167849 & -57.72402211 & -11.55 & 0.05 & -12.45 & 0.04 & -12.21 & 0.07 \\
\hline 6 & 273.4229748 & -57.72139585 & -10.56 & 0.03 & -10.94 & 0.04 & -12.11 & 0.08 \\
\hline 7 & 273.4230317 & -57.72231699 & -9.93 & 0.04 & -10.18 & 0.08 & -12.00 & 0.02 \\
\hline 8 & 273.4240561 & -57.72152951 & -8.93 & 0.09 & -10.29 & 0.05 & -12.24 & 0.09 \\
\hline 9 & 273.4126981 & -57.72621106 & -12.88 & 0.05 & -13.10 & 0.03 & -13.35 & 0.03 \\
\hline 10 & 273.4127251 & -57.72525032 & -11.08 & 0.09 & -11.83 & 0.12 & -11.87 & 0.10 \\
\hline 11 & 273.4124913 & -57.72636619 & -11.88 & 0.02 & -12.08 & 0.04 & -12.71 & 0.05 \\
\hline 12 & 273.4126885 & -57.72499269 & -12.73 & 0.05 & -13.52 & 0.03 & -13.37 & 0.02 \\
\hline 13 & 273.4126222 & -57.72421981 & -10.61 & 0.05 & -11.47 & 0.03 & -11.90 & 0.10 \\
\hline 14 & 273.4126044 & -57.72447105 & -10.59 & 0.06 & -12.28 & 0.02 & -12.28 & 0.07 \\
\hline 15 & 273.4122004 & -57.72543793 & -11.09 & 0.13 & -11.61 & 0.11 & -11.99 & 0.09 \\
\hline 16 & 273.4120619 & -57.72591473 & -12.36 & 0.02 & -13.28 & 0.02 & -12.76 & 0.04 \\
\hline 17 & 273.4118376 & -57.72547113 & -11.04 & 0.15 & -11.88 & 0.09 & -12.41 & 0.06 \\
\hline 18 & 273.4107751 & -57.72651001 & -11.29 & 0.05 & -11.13 & 0.09 & -13.45 & 0.05 \\
\hline
\end{tabular}

Table 44

Derived Properties of Star Clusters in IC 4687N

\begin{tabular}{|c|c|c|c|c|c|c|}
\hline ID & $\log ($ Age $)$ & $\sigma_{\text {Age }}$ & $\log \left(M / M_{\odot}\right)$ & $\sigma_{M}$ & $A_{V}$ & $\sigma_{A_{V}}$ \\
\hline 1 & 6.94 & 0.01 & 3.98 & 0.22 & 0.01 & 0.03 \\
\hline 2 & 7.76 & 0.43 & 7.13 & 0.35 & 1.80 & 0.30 \\
\hline 3 & 6.66 & 0.09 & 6.37 & 0.27 & 1.00 & 0.19 \\
\hline 4 & 7.65 & 0.37 & 7.09 & 0.31 & 1.10 & 0.25 \\
\hline 5 & 8.01 & 0.75 & 6.16 & 0.58 & 0.50 & 0.59 \\
\hline 6 & 6.36 & 0.35 & 5.39 & 0.37 & 1.50 & 0.32 \\
\hline 7 & 6.72 & 0.45 & 4.41 & 0.37 & 0.50 & 0.32 \\
\hline 8 & 6.94 & 0.01 & 4.15 & 0.20 & 0.01 & 0.01 \\
\hline 9 & 6.66 & 0.01 & 6.10 & 0.18 & 1.50 & 0.53 \\
\hline 10 & 6.00 & 0.85 & 5.94 & 0.66 & 2.10 & 0.69 \\
\hline 11 & 6.66 & 0.31 & 5.60 & 0.18 & 1.30 & 0.05 \\
\hline 12 & 6.70 & 0.97 & 6.05 & 0.70 & 1.50 & 0.75 \\
\hline 13 & 7.74 & 0.38 & 5.58 & 0.33 & 0.40 & 0.27 \\
\hline 14 & 7.65 & 0.01 & 5.36 & 0.20 & 0.10 & 0.08 \\
\hline 15 & 6.68 & 0.66 & 5.28 & 0.62 & 1.30 & 0.63 \\
\hline 16 & 8.06 & 0.86 & 6.57 & 0.70 & 0.60 & 0.76 \\
\hline 17 & 6.80 & 0.48 & 5.20 & 0.45 & 1.00 & 0.39 \\
\hline 18 & 6.66 & 0.09 & 4.88 & 0.24 & 0.40 & 0.15 \\
\hline
\end{tabular}

Table 45

Observed Properties of Star Clusters in IC 4687S

\begin{tabular}{lccccccc}
\hline \hline ID & R.A. & Decl. & $M_{B}$ & $\sigma_{B}$ & $M_{I}$ & $\sigma_{I}$ & $M_{\mathrm{FUV}}$ \\
\hline 1 & 273.4156651 & -57.7476378 & -9.47 & 0.16 & -11.63 & 0.09 & -10.47 \\
2 & 273.4174919 & -57.74709814 & -12.71 & 0.02 & -13.50 & 0.01 & -13.60 \\
3 & 273.4181417 & -57.74844252 & -13.11 & 0.02 & -14.08 & 0.04 & -12.93 \\
4 & 273.4184439 & -57.74809628 & -12.50 & 0.05 & -13.27 & 0.07 & 0.02 \\
5 & 273.4192425 & -57.74811065 & -11.58 & 0.05 & -12.54 & 0.03 & -11.14 \\
\hline
\end{tabular}


Table 46

Derived Properties of Star Clusters in IC 4687S

\begin{tabular}{lcccccc}
\hline \hline ID & Log(Age) & $\sigma_{\text {Age }}$ & $\log \left(M / M_{\odot}\right)$ & $\sigma_{M}$ & $A_{V}$ \\
\hline 1 & 6.94 & 0.11 & 5.16 & 0.37 & 0.32 & 0.28 \\
2 & 6.72 & 0.34 & 5.94 & 0.66 & 0.30 \\
3 & 8.41 & 0.66 & 6.94 & 0.19 & 0.30 \\
4 & 8.66 & 0.03 & 6.75 & 0.73 & 0.01 \\
5 & 6.40 & 0.76 & 6.19 & 0.70 & 0.05 \\
& & & & 0.79 \\
\hline
\end{tabular}

Table 47

Observed Properties of Star Clusters in NGC 6786

\begin{tabular}{|c|c|c|c|c|c|c|c|c|}
\hline ID & R.A. & Decl. & $M_{B}$ & $\sigma_{B}$ & $M_{I}$ & $\sigma_{I}$ & $M_{\mathrm{FUV}}$ & $\sigma_{\mathrm{FUV}}$ \\
\hline 1 & 287.7197193 & 73.41100197 & -10.99 & 0.02 & -11.66 & 0.03 & -12.39 & 0.05 \\
\hline 2 & 287.719232 & 73.40994657 & -12.43 & 0.02 & -12.93 & 0.04 & -13.98 & 0.03 \\
\hline 3 & 287.7238745 & 73.41165138 & -10.79 & 0.04 & -11.30 & 0.04 & -12.09 & 0.07 \\
\hline 4 & 287.7178111 & 73.40815357 & -9.46 & 0.06 & -10.03 & 0.06 & -10.95 & 0.22 \\
\hline 5 & 287.7238495 & 73.41044623 & -11.77 & 0.07 & -12.37 & 0.14 & -11.97 & 0.08 \\
\hline 6 & 287.7234966 & 73.41051976 & -13.43 & 0.02 & -13.41 & 0.06 & -14.47 & 0.02 \\
\hline 7 & 287.7229265 & 73.41018561 & -15.39 & 0.01 & -15.31 & 0.01 & -17.41 & 0.01 \\
\hline 8 & 287.7227232 & 73.41040649 & -15.05 & 0.01 & -15.31 & 0.01 & -16.32 & 0.01 \\
\hline 9 & 287.7229402 & 73.41032417 & -14.37 & 0.02 & -14.51 & 0.03 & -16.37 & 0.01 \\
\hline 10 & 287.7267694 & 73.41270096 & -9.77 & 0.18 & -11.79 & 0.04 & -10.19 & 0.11 \\
\hline 11 & 287.7250088 & 73.41164334 & -10.13 & 0.06 & -10.10 & 0.11 & -11.64 & 0.11 \\
\hline 12 & 287.7242707 & 73.4107057 & -12.76 & 0.03 & -13.56 & 0.04 & -13.56 & 0.02 \\
\hline 13 & 287.7250077 & 73.41029358 & -14.21 & 0.03 & -13.58 & 0.10 & -15.98 & 0.01 \\
\hline 14 & 287.7251949 & 73.41061029 & -12.67 & 0.06 & -13.27 & 0.10 & -12.80 & 0.04 \\
\hline 15 & 287.7254972 & 73.41090859 & -10.45 & 0.06 & -10.27 & 0.20 & -12.33 & 0.02 \\
\hline 16 & 287.7241289 & 73.40990629 & -12.92 & 0.02 & -14.11 & 0.05 & -11.81 & 0.09 \\
\hline 17 & 287.7290544 & 73.4121962 & -11.94 & 0.03 & -12.30 & 0.03 & -13.00 & 0.03 \\
\hline 18 & 287.7242387 & 73.40883366 & -10.24 & 0.04 & -10.27 & 0.08 & -11.97 & 0.08 \\
\hline 19 & 287.7279844 & 73.41092482 & -11.98 & 0.02 & -12.74 & 0.03 & -13.44 & 0.02 \\
\hline 20 & 287.7277016 & 73.41036998 & -12.38 & 0.02 & -12.91 & 0.02 & -14.04 & 0.01 \\
\hline 21 & 287.7287715 & 73.41090241 & -13.47 & 0.01 & -13.63 & 0.01 & -15.31 & 0.01 \\
\hline 22 & 287.7283128 & 73.41059111 & -11.42 & 0.03 & -11.78 & 0.04 & -13.07 & 0.03 \\
\hline 23 & 287.7235043 & 73.40730799 & -11.63 & 0.05 & -12.67 & 0.04 & -12.96 & 0.03 \\
\hline 24 & 287.7270499 & 73.40763824 & -11.77 & 0.01 & -12.55 & 0.02 & -12.66 & 0.04 \\
\hline 25 & 287.7264275 & 73.40713593 & -10.01 & 0.07 & -10.54 & 0.10 & -12.16 & 0.07 \\
\hline 26 & 287.7276317 & 73.40724684 & -11.46 & 0.04 & -11.93 & 0.03 & -12.38 & 0.06 \\
\hline 27 & 287.727598 & 73.40748488 & -12.61 & 0.01 & -12.56 & 0.02 & -14.39 & 0.01 \\
\hline 28 & 287.7297973 & 73.40754253 & -10.67 & 0.03 & -11.23 & 0.03 & -12.25 & 0.06 \\
\hline 29 & 287.7327209 & 73.40798896 & -13.58 & 0.01 & -13.70 & 0.01 & -15.19 & 0.01 \\
\hline 30 & 287.7322458 & 73.40744712 & -9.67 & 0.04 & -9.97 & 0.07 & -11.70 & 0.20 \\
\hline 31 & 287.7343067 & 73.40807688 & -11.58 & 0.02 & -11.66 & 0.04 & -13.14 & 0.03 \\
\hline 32 & 287.7349114 & 73.40817718 & -11.63 & 0.04 & -11.99 & 0.05 & -13.11 & 0.06 \\
\hline
\end{tabular}

identified in this galaxy are given in Tables 47 and 48, respectively.

\section{IRAS $20351+2521$}

IRAS $20351+2521$ is an early-stage merger containing multiple star clusters in the northern region where the spiral arms diffuse into multiple components beyond the inner $\sim 5^{\prime \prime}$ ( $4 \mathrm{kpc}$; Figure 51). The maximum $A_{V}$ adopted for this galaxy is 4.7 mag of visual extinction, which is lower than the cited value of 9.4 mag, but prevents our model from predicting masses unrealistically high for even the most massive young star clusters found in the sample. (see Figure 52; Stierwalt et al. 2013). The observed and derived properties for the star clusters identified in this galaxy are given in Tables 49 and 50, respectively.

\section{Zw 096}

Inami et al. (2010) discusses the detailed morphology of this galaxy at length. II Zw 096 is a mid-stage merging system. The 
Table 48

Derived Properties of Star Clusters in NGC 6786

\begin{tabular}{|c|c|c|c|c|c|c|}
\hline ID & Log(Age) & $\sigma_{\text {Age }}$ & $\log \left(M / M_{\odot}\right)$ & $\sigma_{M}$ & $A_{V}$ & $\sigma_{A V}$ \\
\hline 1 & 6.78 & 0.49 & 5.48 & 0.39 & 0.90 & 0.35 \\
\hline 2 & 6.74 & 0.56 & 5.97 & 0.43 & 0.80 & 0.40 \\
\hline 3 & 6.22 & 0.67 & 5.41 & 0.50 & 1.70 & 0.48 \\
\hline 4 & 6.74 & 0.55 & 4.84 & 0.38 & 0.90 & 0.33 \\
\hline 5 & 8.41 & 0.07 & 6.64 & 0.23 & 0.01 & 0.13 \\
\hline 6 & 6.66 & 0.01 & 6.45 & 0.17 & 1.10 & 0.46 \\
\hline 7 & 6.66 & 0.34 & 6.97 & 0.17 & 0.50 & 0.01 \\
\hline 8 & 6.66 & 0.01 & 7.10 & 0.17 & 1.00 & 0.11 \\
\hline 9 & 6.44 & 0.06 & 6.57 & 0.21 & 1.10 & 0.11 \\
\hline 10 & 7.00 & 0.17 & 5.91 & 0.26 & 1.70 & 0.16 \\
\hline 11 & 6.66 & 0.01 & 5.03 & 0.20 & 0.80 & 0.07 \\
\hline 12 & 8.01 & 0.08 & 6.99 & 0.22 & 0.40 & 0.13 \\
\hline 13 & 6.66 & 0.04 & 6.50 & 0.18 & 0.50 & 0.05 \\
\hline 14 & 8.41 & 0.04 & 7.00 & 0.20 & 0.01 & 0.07 \\
\hline 15 & 6.66 & 0.04 & 5.05 & 0.23 & 0.60 & 0.13 \\
\hline 16 & 8.51 & 0.05 & 7.44 & 0.23 & 0.50 & 0.15 \\
\hline 17 & 6.66 & 0.57 & 5.86 & 0.43 & 1.20 & 0.40 \\
\hline 18 & 6.66 & 0.03 & 5.02 & 0.21 & 0.70 & 0.10 \\
\hline 19 & 7.72 & 0.44 & 6.47 & 0.35 & 0.30 & 0.30 \\
\hline 20 & 7.86 & 0.55 & 6.55 & 0.40 & 0.01 & 0.36 \\
\hline 21 & 6.48 & 0.04 & 6.43 & 0.22 & 1.10 & 0.12 \\
\hline 22 & 5.10 & 0.04 & 5.55 & 0.19 & 1.50 & 0.07 \\
\hline 23 & 7.63 & 0.24 & 6.37 & 0.25 & 0.50 & 0.16 \\
\hline 24 & 6.72 & 0.05 & 5.84 & 0.18 & 1.30 & 0.06 \\
\hline 25 & 6.84 & 0.35 & 4.91 & 0.27 & 0.40 & 0.19 \\
\hline 26 & 6.66 & 0.05 & 5.67 & 0.18 & 1.30 & 0.02 \\
\hline 27 & 6.66 & 0.35 & 5.97 & 0.18 & 0.70 & 0.03 \\
\hline 28 & 6.74 & 0.57 & 5.27 & 0.43 & 0.80 & 0.40 \\
\hline 29 & 6.66 & 0.29 & 6.41 & 0.17 & 0.80 & 0.04 \\
\hline 30 & 6.72 & 0.05 & 4.70 & 0.20 & 0.50 & 0.09 \\
\hline 31 & 6.66 & 0.58 & 5.61 & 0.18 & 0.80 & 0.04 \\
\hline 32 & 6.40 & 0.05 & 5.67 & 0.20 & 1.50 & 0.08 \\
\hline
\end{tabular}

Table 49

Observed Properties of Star Clusters in IRAS 20351+2521

\begin{tabular}{|c|c|c|c|c|c|c|c|c|}
\hline ID & R.A. & Decl. & $M_{B}$ & $\sigma_{B}$ & $M_{I}$ & $\sigma_{I}$ & $M_{\mathrm{FUV}}$ & $\sigma_{\mathrm{FUV}}$ \\
\hline 1 & 309.3189111 & 25.52810092 & -11.77 & 0.04 & -11.99 & 0.06 & -12.96 & 0.25 \\
\hline 2 & 309.3201669 & 25.53148902 & -12.10 & 0.03 & -12.78 & 0.03 & -13.40 & 0.14 \\
\hline 3 & 309.3204655 & 25.53148786 & -14.20 & 0.01 & -14.34 & 0.02 & -15.85 & 0.04 \\
\hline 4 & 309.3207482 & 25.52879944 & -11.57 & 0.05 & -12.24 & 0.05 & -12.86 & 0.05 \\
\hline 5 & 309.3205846 & 25.52701311 & -12.18 & 0.08 & -11.64 & 0.16 & -12.50 & 0.26 \\
\hline 6 & 309.3209895 & 25.52805392 & -12.80 & 0.04 & -14.63 & 0.01 & -12.35 & 0.24 \\
\hline 7 & 309.3213802 & 25.52804452 & -12.92 & 0.03 & -13.44 & 0.03 & -13.04 & 0.17 \\
\hline 8 & 309.3212834 & 25.52633971 & -11.39 & 0.06 & -12.12 & 0.06 & -12.77 & 0.22 \\
\hline 9 & 309.3215513 & 25.53117662 & -11.92 & 0.07 & -11.68 & 0.12 & -12.49 & 0.16 \\
\hline 10 & 309.3227499 & 25.52581676 & -12.05 & 0.06 & -12.70 & 0.07 & -12.76 & 0.16 \\
\hline 11 & 309.3227896 & 25.52569385 & -12.91 & 0.02 & -12.96 & 0.05 & -13.61 & 0.07 \\
\hline 12 & 309.3232605 & 25.52520015 & -12.72 & 0.05 & -13.34 & 0.06 & -13.80 & 0.12 \\
\hline 13 & 309.3237357 & 25.52539922 & -11.60 & 0.05 & -12.42 & 0.03 & -12.26 & 0.22 \\
\hline 14 & 309.3236793 & 25.52933194 & -11.99 & 0.06 & -12.00 & 0.07 & -13.48 & 0.12 \\
\hline 15 & 309.3256351 & 25.52614986 & -11.60 & 0.08 & -12.04 & 0.10 & -12.83 & 0.04 \\
\hline 16 & 309.3255973 & 25.526354 & -12.15 & 0.05 & -12.24 & 0.08 & -13.38 & 0.06 \\
\hline 17 & 309.3256314 & 25.53101137 & -11.60 & 0.05 & -14.92 & 0.01 & -12.71 & 0.01 \\
\hline 18 & 309.3256579 & 25.53150391 & -11.02 & 0.08 & -11.77 & 0.06 & -13.02 & 0.21 \\
\hline 19 & 309.3259664 & 25.52654554 & -11.17 & 0.15 & -12.53 & 0.06 & -12.95 & 0.27 \\
\hline 20 & 309.3261938 & 25.52631453 & -13.23 & 0.02 & -13.84 & 0.02 & -13.45 & 0.16 \\
\hline 21 & 309.3262881 & 25.52747562 & -13.98 & 0.02 & -14.03 & 0.02 & -16.15 & 0.01 \\
\hline 22 & 309.3262506 & 25.53020748 & -11.03 & 0.05 & -11.65 & 0.05 & -13.08 & 0.03 \\
\hline
\end{tabular}


Table 50

Derived Properties of Star Clusters in IRAS 20351+2521

\begin{tabular}{|c|c|c|c|c|c|c|}
\hline ID & Log(Age) & $\sigma_{\mathrm{Age}}$ & $\log \left(M / M_{\odot}\right)$ & $\sigma_{M}$ & $A_{V}$ & $\sigma_{A_{V}}$ \\
\hline 1 & 6.54 & 0.33 & 5.69 & 0.16 & 1.50 & 0.02 \\
\hline 2 & 6.74 & 0.19 & 5.65 & 0.16 & 1.10 & 0.31 \\
\hline 3 & 6.54 & 0.32 & 6.50 & 0.15 & 1.20 & 0.23 \\
\hline 4 & 7.76 & 0.42 & 5.92 & 0.16 & 0.40 & 0.20 \\
\hline 5 & 6.52 & 0.40 & 6.07 & 0.18 & 1.90 & 0.45 \\
\hline 6 & 6.78 & 0.05 & 6.57 & 0.16 & 2.30 & 0.59 \\
\hline 7 & 8.51 & 0.11 & 6.72 & 0.16 & 0.01 & 0.53 \\
\hline 8 & 7.70 & 0.12 & 5.81 & 0.17 & 0.40 & 0.16 \\
\hline 9 & 6.52 & 0.23 & 5.91 & 0.17 & 1.80 & 0.46 \\
\hline 10 & 8.36 & 0.53 & 6.26 & 0.17 & 0.01 & 0.19 \\
\hline 11 & 6.52 & 0.08 & 6.31 & 0.16 & 1.80 & 0.05 \\
\hline 12 & 6.64 & 0.37 & 6.19 & 0.16 & 1.60 & 0.54 \\
\hline 13 & 7.96 & 0.12 & 6.13 & 0.16 & 0.60 & 0.08 \\
\hline 14 & 6.52 & 0.18 & 5.67 & 0.17 & 1.30 & 0.12 \\
\hline 15 & 6.52 & 0.29 & 5.68 & 0.18 & 1.60 & 0.24 \\
\hline 16 & 6.52 & 0.32 & 5.84 & 0.16 & 1.50 & 0.24 \\
\hline 17 & 8.11 & 0.39 & 6.22 & 0.16 & 0.60 & 0.23 \\
\hline 18 & 6.94 & 0.52 & 5.09 & 0.18 & 0.50 & 0.59 \\
\hline 19 & 7.65 & 0.53 & 5.53 & 0.22 & 0.10 & 0.36 \\
\hline 20 & 6.52 & 0.07 & 6.65 & 0.16 & 2.20 & 0.41 \\
\hline 21 & 6.44 & 0.19 & 6.49 & 0.16 & 1.10 & 0.19 \\
\hline 22 & 7.36 & 0.24 & 5.39 & 0.16 & 0.30 & 0.58 \\
\hline
\end{tabular}

Table 51

Observed Properties of Star Clusters in II Zw 096

\begin{tabular}{|c|c|c|c|c|c|c|c|c|}
\hline ID & R.A. & Decl. & $M_{B}$ & $\sigma_{B}$ & $M_{I}$ & $\sigma_{I}$ & $M_{\mathrm{FUV}}$ & $\sigma_{\mathrm{FUV}}$ \\
\hline 1 & 314.347937 & 17.13209914 & -14.40 & 0.02 & -14.52 & 0.01 & -16.48 & 0.01 \\
\hline 2 & 314.3488605 & 17.12855532 & -13.82 & 0.03 & -14.60 & 0.03 & -15.53 & 0.05 \\
\hline 3 & 314.3490196 & 17.12831079 & -13.91 & 0.02 & -14.50 & 0.05 & -15.42 & 0.02 \\
\hline 4 & 314.3489006 & 17.12844657 & -14.02 & 0.05 & -14.14 & 0.07 & -14.94 & 0.12 \\
\hline 5 & 314.3487591 & 17.13097999 & -13.81 & 0.02 & -13.94 & 0.02 & -15.86 & 0.01 \\
\hline 6 & 314.3491325 & 17.12863715 & -13.77 & 0.02 & -14.84 & 0.01 & -14.42 & 0.03 \\
\hline 7 & 314.349413 & 17.12830611 & -13.18 & 0.02 & -14.06 & 0.02 & -14.27 & 0.04 \\
\hline 8 & 314.3494999 & 17.12879568 & -14.29 & 0.01 & -14.38 & 0.02 & -16.21 & 0.01 \\
\hline 9 & 314.3497151 & 17.12841414 & -16.14 & 0.01 & -16.84 & 0.01 & -17.62 & 0.01 \\
\hline 10 & 314.3501466 & 17.12654531 & -17.92 & 0.01 & -18.33 & 0.01 & -19.48 & 0.01 \\
\hline 11 & 314.3501115 & 17.12664894 & -15.51 & 0.05 & -16.26 & 0.05 & -16.12 & 0.03 \\
\hline 12 & 314.3500502 & 17.12680274 & -13.52 & 0.11 & -13.88 & 0.19 & -14.82 & 0.07 \\
\hline 13 & 314.3501617 & 17.12810109 & -14.29 & 0.02 & -14.84 & 0.02 & -15.64 & 0.02 \\
\hline 14 & 314.3504248 & 17.12671799 & -14.41 & 0.10 & -14.91 & 0.09 & -15.82 & 0.05 \\
\hline 15 & 314.3504765 & 17.12652561 & -16.42 & 0.01 & -16.64 & 0.02 & -17.80 & 0.02 \\
\hline 16 & 314.350505 & 17.12642631 & -16.31 & 0.01 & -16.51 & 0.02 & -17.94 & 0.01 \\
\hline 17 & 314.352852 & 17.12679784 & -14.29 & 0.01 & -14.06 & 0.03 & -16.45 & 0.01 \\
\hline 18 & 314.353755 & 17.1273767 & -13.74 & 0.01 & -13.88 & 0.02 & -15.87 & 0.01 \\
\hline 19 & 314.3538332 & 17.12884574 & -13.71 & 0.01 & -14.39 & 0.01 & -15.76 & 0.01 \\
\hline 20 & 314.3539789 & 17.12839369 & -12.26 & 0.02 & -12.92 & 0.02 & -14.44 & 0.03 \\
\hline
\end{tabular}

western component is a roughly face-on spiral galaxy with star clusters along the spiral arms. The southeast end of the spiral, approximately $11 . " 6(8.4 \mathrm{kpc})$ from the nucleus, contains a distinct cluster-rich region (Figure 53). The maximum $A_{V}$ adopted for this galaxy is 3.0 mag of visual extinction (see Figure 54; Inami et al. 2010). The observed and derived properties for the star clusters identified in this galaxy are given in Tables 51 and 52, respectively. 
Table 52

Derived Properties of Star Clusters in II Zw 096

\begin{tabular}{|c|c|c|c|c|c|c|}
\hline ID & $\log ($ Age $)$ & $\sigma_{\text {Age }}$ & $\log \left(M / M_{\odot}\right)$ & $\sigma_{M}$ & $A_{V}$ & $\sigma_{A_{V}}$ \\
\hline 1 & 6.40 & 0.08 & 6.76 & 0.22 & 1.20 & 0.12 \\
\hline 2 & 7.40 & 0.25 & 6.64 & 0.24 & 0.50 & 0.16 \\
\hline 3 & 6.74 & 0.45 & 6.32 & 0.39 & 1.00 & 0.35 \\
\hline 5 & 6.42 & 0.12 & 6.52 & 0.24 & 1.20 & 0.16 \\
\hline 6 & 7.63 & 0.27 & 7.04 & 0.26 & 1.00 & 0.19 \\
\hline 7 & 7.63 & 0.26 & 6.64 & 0.26 & 0.70 & 0.18 \\
\hline 10 & 5.70 & 0.62 & 8.60 & 0.44 & 1.60 & 0.41 \\
\hline 11 & 6.54 & 0.85 & 7.45 & 0.81 & 2.00 & 0.93 \\
\hline 12 & 6.54 & 0.55 & 6.39 & 0.53 & 1.50 & 0.52 \\
\hline 13 & 6.72 & 0.61 & 6.60 & 0.54 & 1.20 & 0.54 \\
\hline 14 & 6.58 & 0.62 & 6.73 & 0.55 & 1.40 & 0.55 \\
\hline 15 & 6.54 & 0.01 & 7.50 & 0.17 & 1.40 & 0.03 \\
\hline 20 & 6.96 & 0.15 & 5.57 & 0.18 & 0.40 & 0.06 \\
\hline
\end{tabular}

Table 53

Observed Properties of Star Clusters in ESO 148-IG002

\begin{tabular}{cccccccc}
\hline \hline ID & R.A. & Decl. & $M_{B}$ & $\sigma_{B}$ & $M_{I}$ & $\sigma_{I}$ & $M_{\mathrm{FUV}}$ \\
\hline 1 & 348.9465405 & -59.05411541 & -14.15 & 0.01 & -14.90 & 0.01 & -14.43 \\
2 & 348.9468576 & -59.0539657 & -13.60 & 0.02 & -14.73 & 0.02 & -13.26 \\
3 & 348.9462256 & -59.05361789 & -12.73 & 0.03 & -13.39 & 0.04 & -12.63 \\
4 & 348.9469037 & -59.05365887 & -11.95 & 0.09 & -13.24 & 0.08 & -12.87 \\
5 & 348.9454074 & -59.05331294 & -13.54 & 0.15 & -14.19 & 0.09 & -14.48 \\
6 & 348.9468404 & -59.05307144 & -14.27 & 0.02 & -15.05 & 0.01 & -14.84 \\
7 & 348.9456965 & -59.05497655 & -14.45 & 0.04 & -15.22 & 0.06 & 0.03 \\
\hline
\end{tabular}

Table 54

Derived Properties of Star Clusters in ESO 148-IG002

\begin{tabular}{lccccc}
\hline \hline ID & $\log ($ Age $)$ & $\sigma_{\text {Age }}$ & $\log \left(M / M_{\odot}\right)$ & $\sigma_{M}$ & $A_{V}$ \\
\hline 1 & 8.46 & 0.03 & 7.17 & 0.16 & 0.01 \\
2 & 8.41 & 0.40 & 7.23 & 0.45 & 0.60 \\
3 & 8.61 & 0.06 & 6.73 & 0.17 & 0.01 \\
4 & 7.65 & 0.25 & 6.21 & 0.24 & 0.43 \\
5 & 8.26 & 0.79 & 6.80 & 0.69 & 0.02 \\
6 & 6.48 & 0.90 & 7.13 & 0.84 & 0.17 \\
7 & 8.56 & 0.04 & 7.38 & 0.17 & 0.14 \\
\hline
\end{tabular}

\section{ESO 148-IG002}

ESO 148-IG002 is a late-stage merger with a projected nuclear separation of $\sim 4$." $7(4.2 \mathrm{kpc})$. The galaxy has a series of bright clusters which lie along a north-south ridge to the east of the bulge (Figure 55). The maximum $A_{V}$ adopted for this galaxy is $2.5 \mathrm{mag}$ of visual extinction (see Figure 56; Johansson \& Bergvall 1988). The observed and derived properties for the star clusters identified in this galaxy are given in Tables 53 and 54, respectively.

\section{NGC 7674}

NGC 7674 an early-stage merger with a face-on spiral galaxy and companions to the northeast and southeast. Star clusters are visible along the prominent spiral arms throughout the galaxy (Figure 57). The maximum $A_{V}$ adopted for this galaxy is 2.0 mag of visual extinction (see Figure 58; Momjian et al. 2003). The observed and derived properties for the star clusters identified in this galaxy are given in Tables 55 and 56, respectively. 
Table 55

Observed Properties of Star Clusters in NGC 7674

\begin{tabular}{|c|c|c|c|c|c|c|c|c|}
\hline ID & R.A. & Decl. & $M_{B}$ & $\sigma_{B}$ & $M_{I}$ & $\sigma_{I}$ & $M_{\mathrm{FUV}}$ & $\sigma_{\mathrm{FUV}}$ \\
\hline 1 & 351.9831239 & 8.778349264 & -12.20 & 0.02 & -12.57 & 0.03 & -14.06 & 0.02 \\
\hline 2 & 351.9849596 & 8.772096618 & -11.55 & 0.02 & -11.73 & 0.04 & -13.17 & 0.05 \\
\hline 3 & 351.9850026 & 8.772116709 & -11.44 & 0.02 & -11.76 & 0.03 & -12.79 & 0.08 \\
\hline 4 & 351.9832985 & 8.779441129 & -10.77 & 0.11 & -11.11 & 0.13 & -13.04 & 0.06 \\
\hline 5 & 351.9830552 & 8.780545473 & -10.91 & 0.03 & -11.08 & 0.05 & -12.85 & 0.04 \\
\hline 6 & 351.9840117 & 8.778513551 & -12.29 & 0.04 & -12.74 & 0.03 & -13.63 & 0.04 \\
\hline 7 & 351.9849762 & 8.780046055 & -12.87 & 0.02 & -12.92 & 0.03 & -15.07 & 0.02 \\
\hline 8 & 351.9855017 & 8.779670342 & -12.42 & 0.04 & -12.61 & 0.09 & -13.89 & 0.06 \\
\hline 9 & 351.9862949 & 8.779049017 & -17.60 & 0.01 & -18.76 & 0.01 & -19.06 & 0.01 \\
\hline 10 & 351.9882912 & 8.773938539 & -12.62 & 0.01 & -13.32 & 0.01 & -14.50 & 0.02 \\
\hline 11 & 351.9885463 & 8.773640234 & -11.47 & 0.03 & -12.12 & 0.05 & -13.84 & 0.03 \\
\hline 12 & 351.9857724 & 8.780029971 & -11.03 & 0.05 & -10.37 & 0.20 & -12.96 & 0.07 \\
\hline 13 & 351.9869669 & 8.777906039 & -11.45 & 0.04 & -11.50 & 0.06 & -12.83 & 0.05 \\
\hline 14 & 351.9868119 & 8.778402264 & -13.02 & 0.01 & -13.05 & 0.03 & -14.91 & 0.01 \\
\hline 15 & 351.9875291 & 8.777102907 & -13.70 & 0.03 & -13.66 & 0.03 & -15.37 & 0.02 \\
\hline 16 & 351.9886887 & 8.774122684 & -11.76 & 0.03 & -11.76 & 0.06 & -14.04 & 0.05 \\
\hline 17 & 351.9874886 & 8.7769875 & -11.39 & 0.19 & -12.26 & 0.16 & -14.14 & 0.04 \\
\hline 18 & 351.9864376 & 8.77992672 & -11.09 & 0.04 & -11.46 & 0.07 & -13.01 & 0.06 \\
\hline 19 & 351.9873013 & 8.778022066 & -10.96 & 0.06 & -11.33 & 0.13 & -12.90 & 0.07 \\
\hline 20 & 351.9867238 & 8.779687958 & -12.50 & 0.03 & -12.67 & 0.04 & -14.62 & 0.01 \\
\hline 21 & 351.9877438 & 8.778029429 & -12.90 & 0.02 & -13.24 & 0.04 & -14.16 & 0.02 \\
\hline 22 & 351.988047 & 8.779603842 & -10.36 & 0.13 & -11.65 & 0.09 & -13.07 & 0.03 \\
\hline 23 & 351.9895457 & 8.777038636 & -10.67 & 0.05 & -10.77 & 0.09 & -12.88 & 0.07 \\
\hline 24 & 351.9886045 & 8.779833873 & -12.05 & 0.03 & -12.33 & 0.05 & -13.70 & 0.03 \\
\hline 25 & 351.9823723 & 8.778916581 & -11.27 & 0.03 & -11.67 & 0.04 & -12.86 & 0.07 \\
\hline
\end{tabular}

Table 56

Derived Properties of Star Clusters in NGC 7674

\begin{tabular}{|c|c|c|c|c|c|c|}
\hline ID & Log(Age) & $\sigma_{\mathrm{Age}}$ & $\log \left(M / M_{\odot}\right)$ & $\sigma_{M}$ & $A_{V}$ & $\sigma_{A_{V}}$ \\
\hline 1 & 6.74 & 0.03 & 5.48 & 0.21 & 0.70 & 0.11 \\
\hline 2 & 6.52 & 0.02 & 5.50 & 0.19 & 1.30 & 0.07 \\
\hline 3 & 6.52 & 0.03 & 5.56 & 0.19 & 1.50 & 0.08 \\
\hline 4 & 6.72 & 0.28 & 4.87 & 0.27 & 0.60 & 0.17 \\
\hline 5 & 6.52 & 0.08 & 5.14 & 0.22 & 1.10 & 0.13 \\
\hline 6 & 6.44 & 0.10 & 6.14 & 0.24 & 1.70 & 0.16 \\
\hline 7 & 6.42 & 0.10 & 6.09 & 0.22 & 1.10 & 0.14 \\
\hline 8 & 6.52 & 0.05 & 5.90 & 0.20 & 1.40 & 0.09 \\
\hline 9 & 6.80 & 0.03 & 7.78 & 0.17 & 0.90 & 0.03 \\
\hline 10 & 7.00 & 0.07 & 5.97 & 0.17 & 0.70 & 0.04 \\
\hline 11 & 7.00 & 0.10 & 5.46 & 0.17 & 0.60 & 1.12 \\
\hline 12 & 6.52 & 0.01 & 5.08 & 0.20 & 0.90 & 0.09 \\
\hline 13 & 6.52 & 0.01 & 5.51 & 0.18 & 1.40 & 0.04 \\
\hline 14 & 6.52 & 0.01 & 5.98 & 0.17 & 1.10 & 0.03 \\
\hline 15 & 6.52 & 0.01 & 6.31 & 0.17 & 1.20 & 0.03 \\
\hline 16 & 6.50 & 0.08 & 5.39 & 0.22 & 0.90 & 0.13 \\
\hline 17 & 7.00 & 0.43 & 5.43 & 0.27 & 0.60 & 0.06 \\
\hline 18 & 6.74 & 0.18 & 5.04 & 0.27 & 0.70 & 0.20 \\
\hline 19 & 6.74 & 0.19 & 4.98 & 0.29 & 0.70 & 0.22 \\
\hline 20 & 6.58 & 0.27 & 5.70 & 0.27 & 0.90 & 0.20 \\
\hline 21 & 6.54 & 0.01 & 6.14 & 0.18 & 1.50 & 0.05 \\
\hline 22 & 6.82 & 0.10 & 4.76 & 0.22 & 0.60 & 1.12 \\
\hline 23 & 6.68 & 0.11 & 4.90 & 0.24 & 0.70 & 0.16 \\
\hline 24 & 6.52 & 0.28 & 5.70 & 0.24 & 1.30 & 0.16 \\
\hline 25 & 6.66 & 0.30 & 5.41 & 0.30 & 1.20 & 0.23 \\
\hline
\end{tabular}




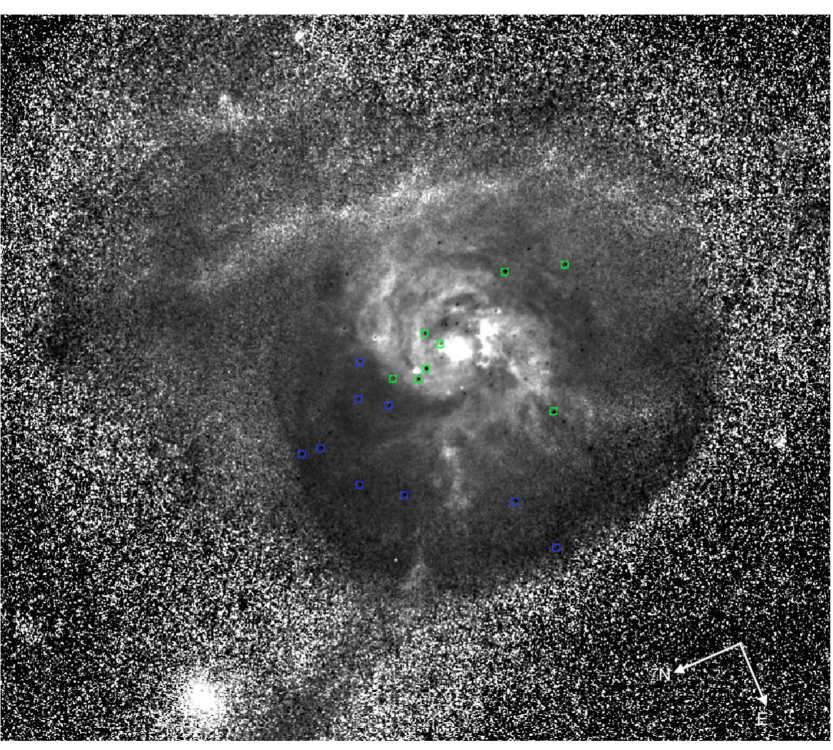

Figure 7. Inverted black and white $B-I$ image of NGC 0017 taken with $H S T$ ACS/WFC F814W and F435W. The bright emission corresponds to redder (i.e., dustier) regions of the galaxy. The blue centroids correspond to clusters found in relatively "dust-free" regions of these galaxies, whereas the green centroids correspond to clusters found in relatively dustier regions of the galaxy.

NGC0017 Color-Color Plot

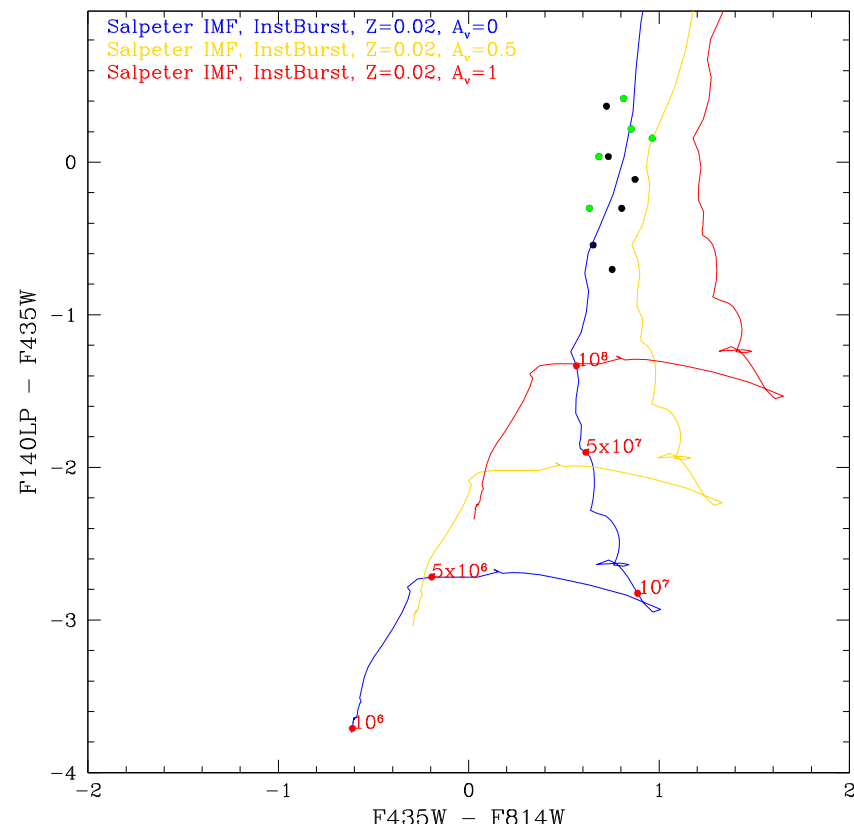

Figure 8. Color-color plot of all star clusters identified in NGC 0017 in F814W, F435W, and F140LP plotted against SSP models with various amounts of visual extinction. The green points correspond to the clusters found in dustier regions of the galaxy in Figure 7.

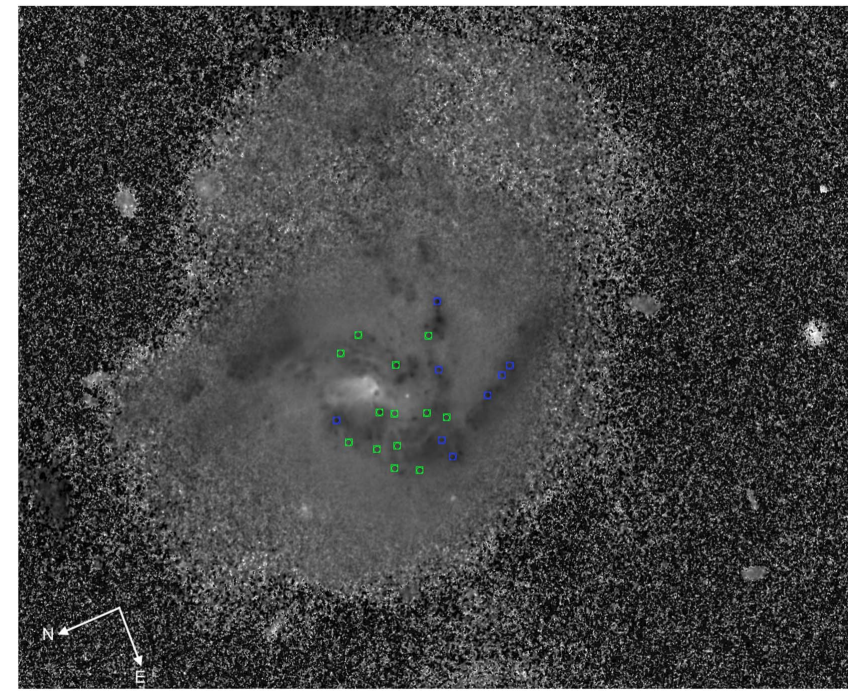

Figure 9. Inverted black and white $B-I$ image of Arp $256 \mathrm{~S}$ taken with $H S T$ ACS/WFC F814W and F435W. The bright emission corresponds to redder (i.e., dustier) regions of the galaxy. The blue centroids correspond to clusters found in relatively "dust-free" regions of these galaxies, whereas the green centroids correspond to clusters found in relatively dustier regions of the galaxy.

ARP256 Color-Color Plot ----

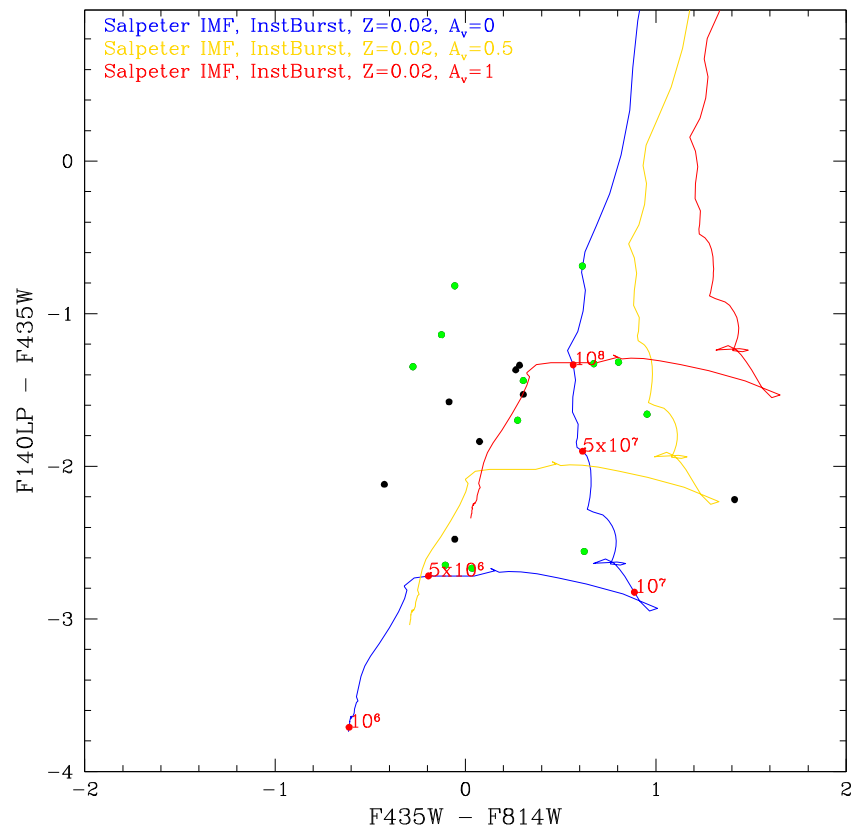

Figure 10. Color-color plot of all star clusters identified in Arp 256S in F814W, F435W, and F140LP plotted against SSP models with various amounts of visual extinction. The green points correspond to the clusters found in dustier regions of the galaxy in Figure 9. 


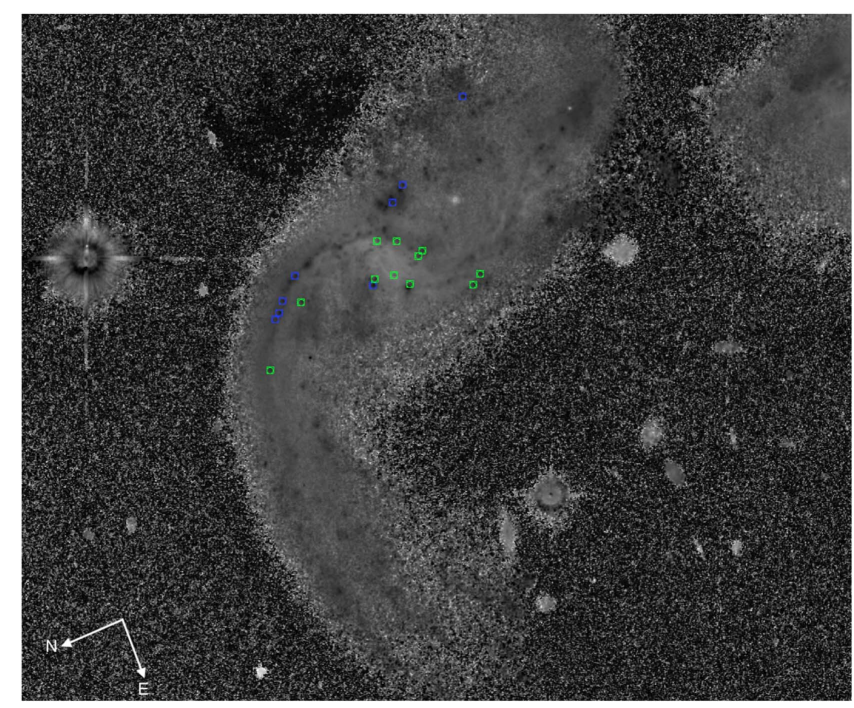

Figure 11. Inverted black and white $B-I$ image of Arp $256 \mathrm{~N}$ taken with $H S T$ ACS/WFC F814W and F435W. The bright emission corresponds to redder (i.e., dustier) regions of the galaxy. The blue centroids correspond to clusters found in relatively "dust-free" regions of these galaxies, whereas the green centroids correspond to clusters found in relatively dustier regions of the galaxy.

ARP256 Color-Color Plot

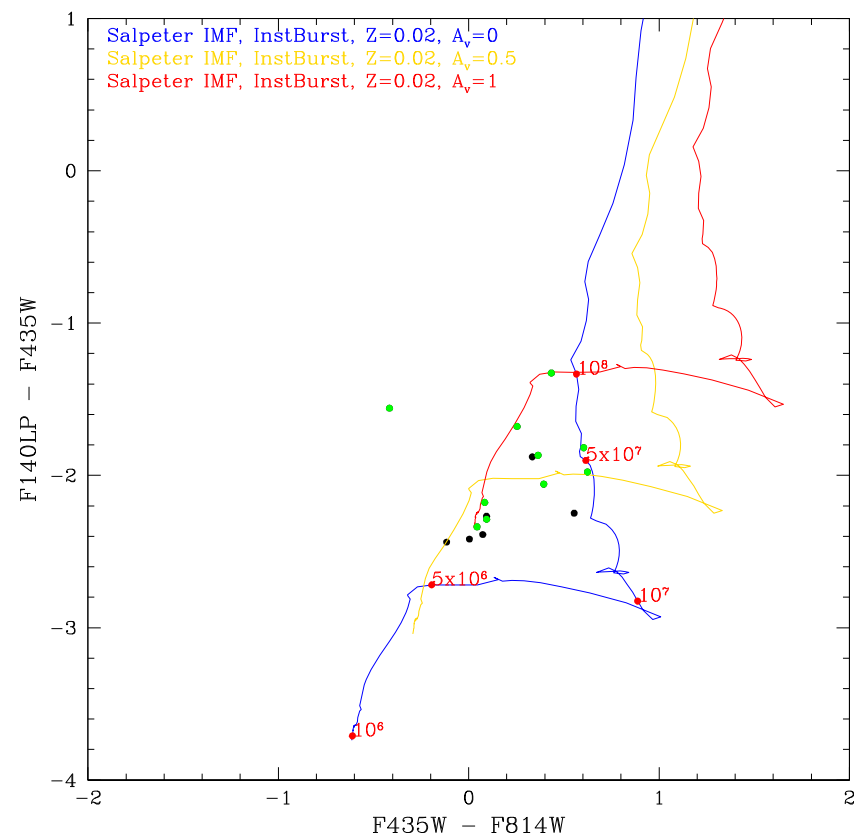

Figure 12. Color-color plot of all star clusters identified in Arp $256 \mathrm{~N}$ in F814W, F435W, and F140LP plotted against SSP models with various amounts of visual extinction. The green points correspond to the clusters found in dustier regions of the galaxy in Figure 11.

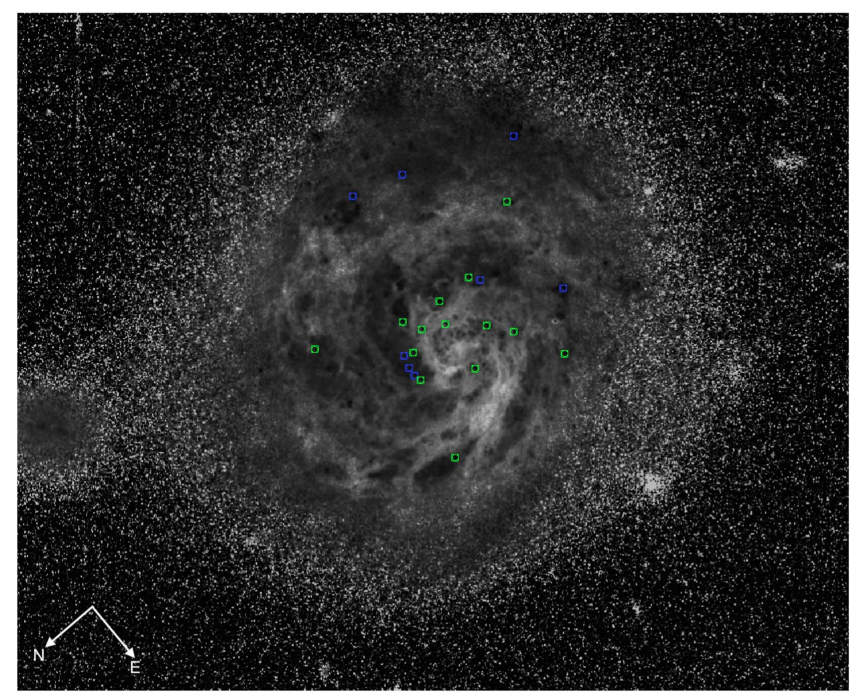

Figure 13. Inverted black and white $B-I$ image of NGC 0695 taken with $H S T$ ACS/WFC F814W and F435W. The bright emission corresponds to redder (i.e., dustier) regions of the galaxy. The blue centroids correspond to clusters found in relatively "dust-free" regions of these galaxies, whereas the green centroids correspond to clusters found in relatively dustier regions of the galaxy.

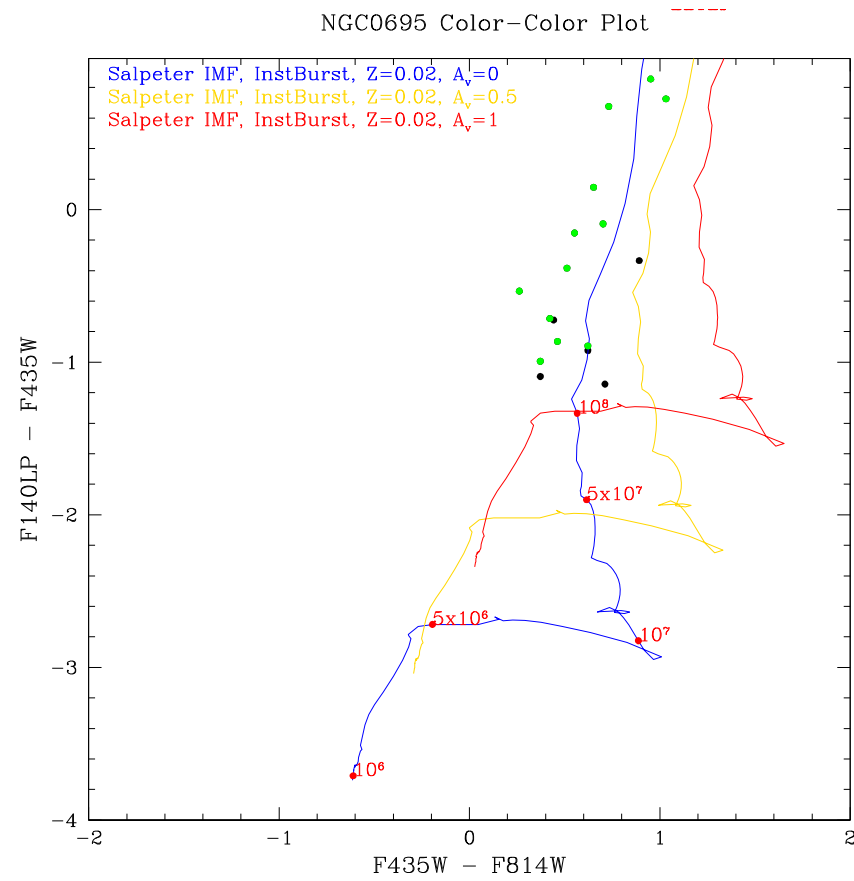

Figure 14. Color-color plot of all star clusters identified in NGC 0695 in F814W, F435W, and F140LP plotted against SSP models with various amounts of visual extinction. The green points correspond to the clusters found in dustier regions of the galaxy in Figure 13. 


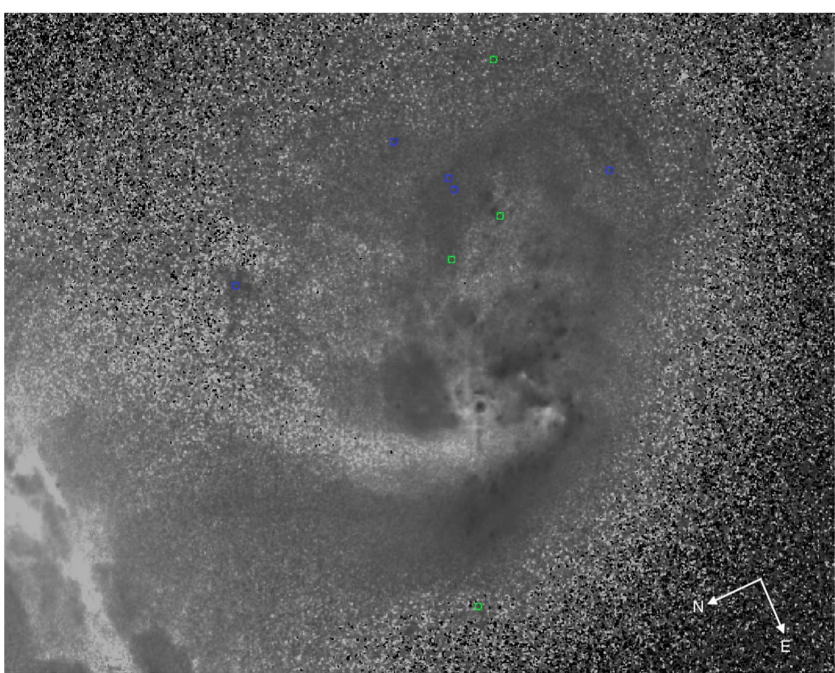

Figure 15. Inverted black and white $B-I$ image of UGC 02369 taken with $H S T$ ACS/WFC F814W and F435W. The bright emission corresponds to redder (i.e., dustier) regions of the galaxy. The blue centroids correspond to clusters found in relatively "dust-free" regions of these galaxies, whereas the green centroids correspond to clusters found in relatively dustier regions of the galaxy.

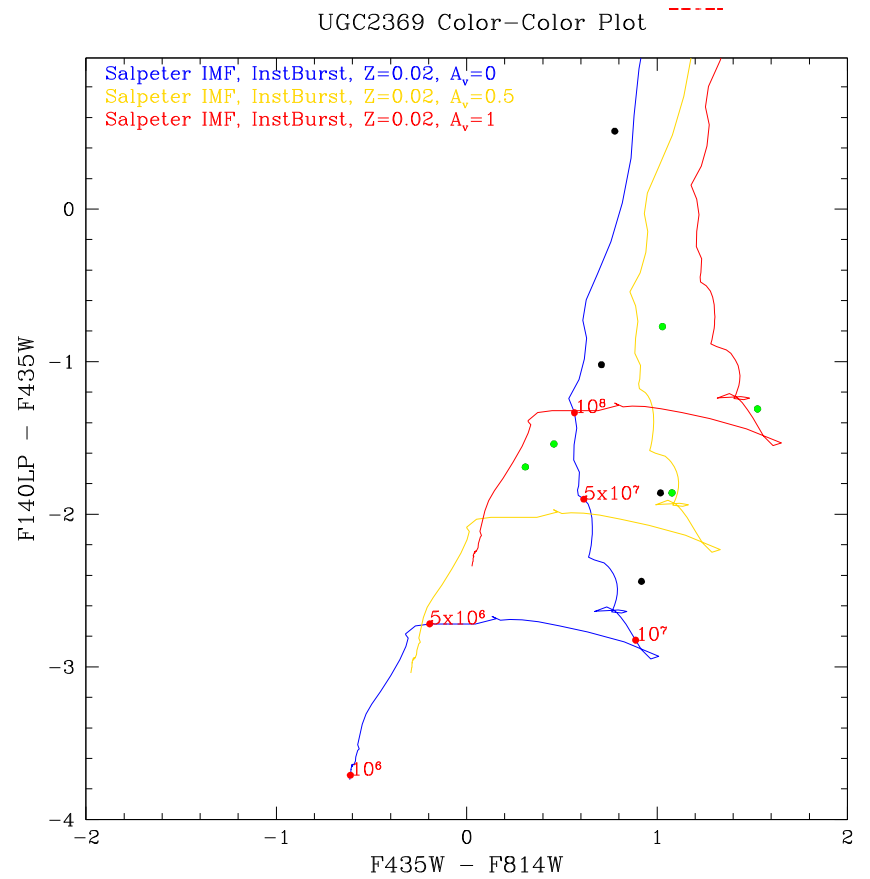

Figure 16. Color-color plot of all star clusters identified in UGC 02369 in F814W, F435W, and F140LP plotted against SSP models with various amounts of visual extinction. The green points correspond to the clusters found in dustier regions of the galaxy in Figure 15.

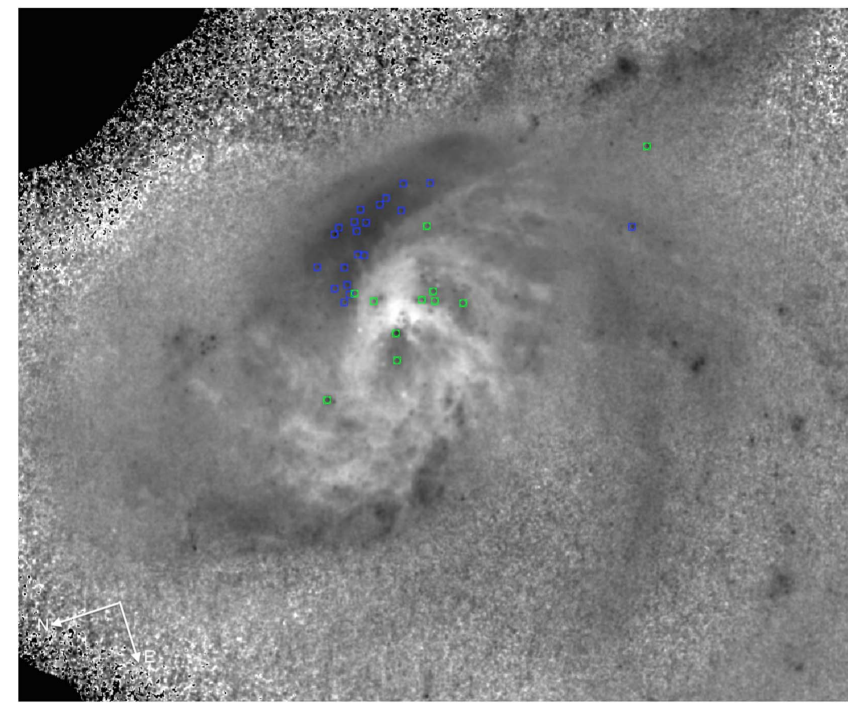

Figure 17. Inverted black and white $B-I$ image of NGC 1614 taken with $H S T$ ACS/WFC F814W and F435W. The bright emission corresponds to redder (i.e., dustier) regions of the galaxy. The blue centroids correspond to clusters found in relatively "dust-free" regions of these galaxies, whereas the green centroids correspond to clusters found in relatively dustier regions of the galaxy.

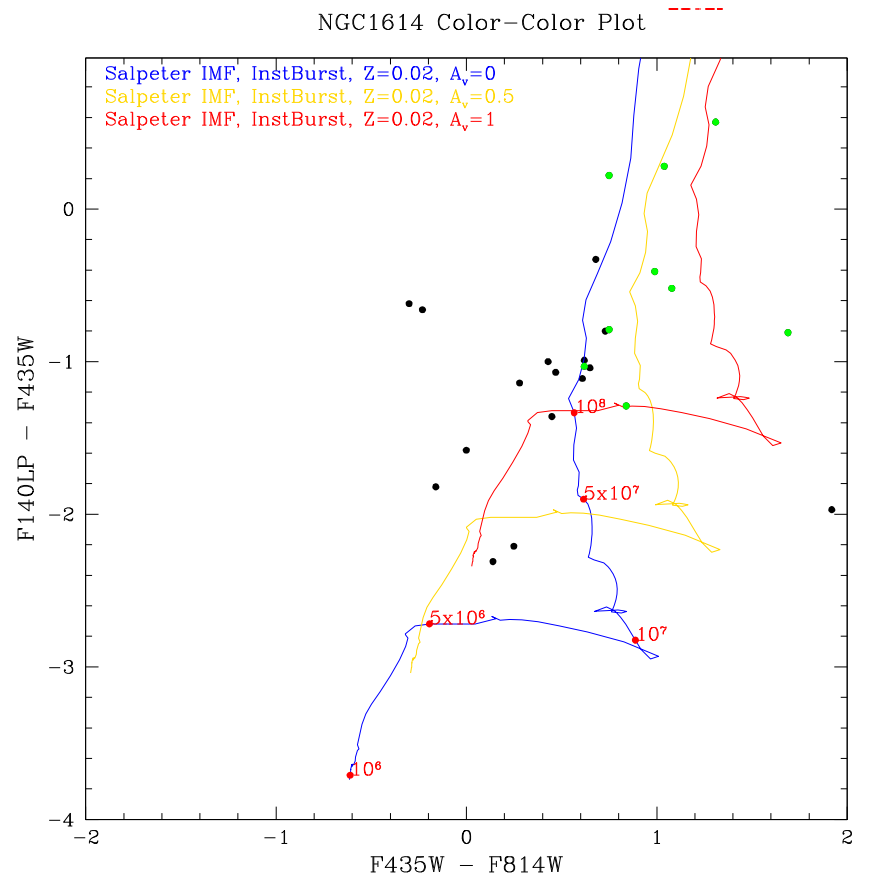

Figure 18. Color-color plot of all star clusters identified in NGC 1614 in F814W, F435W, and F140LP plotted against SSP models with various amounts of visual extinction. The green points correspond to the clusters found in dustier regions of the galaxy in Figure 17. 


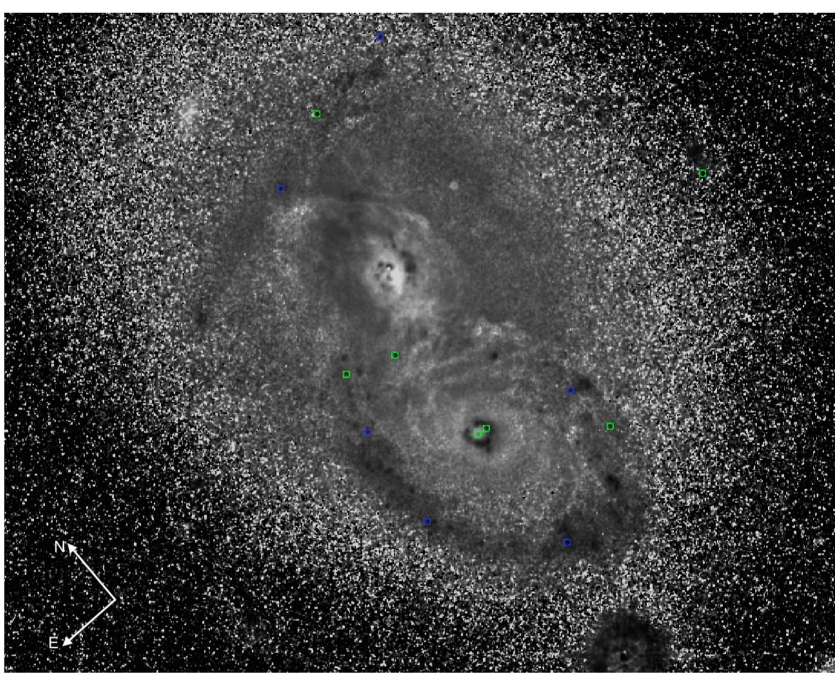

Figure 19. Inverted black and white $B-I$ image of 2MASX J060945822140234 taken with HST ACS/WFC F814W and F435W. The bright emission corresponds to redder (i.e., dustier) regions of the galaxy. The blue centroids correspond to clusters found in relatively "dust-free" regions of these galaxies, whereas the green centroids correspond to clusters found in relatively dustier regions of the galaxy.

2MASSJ06094582 Color-Color Plot

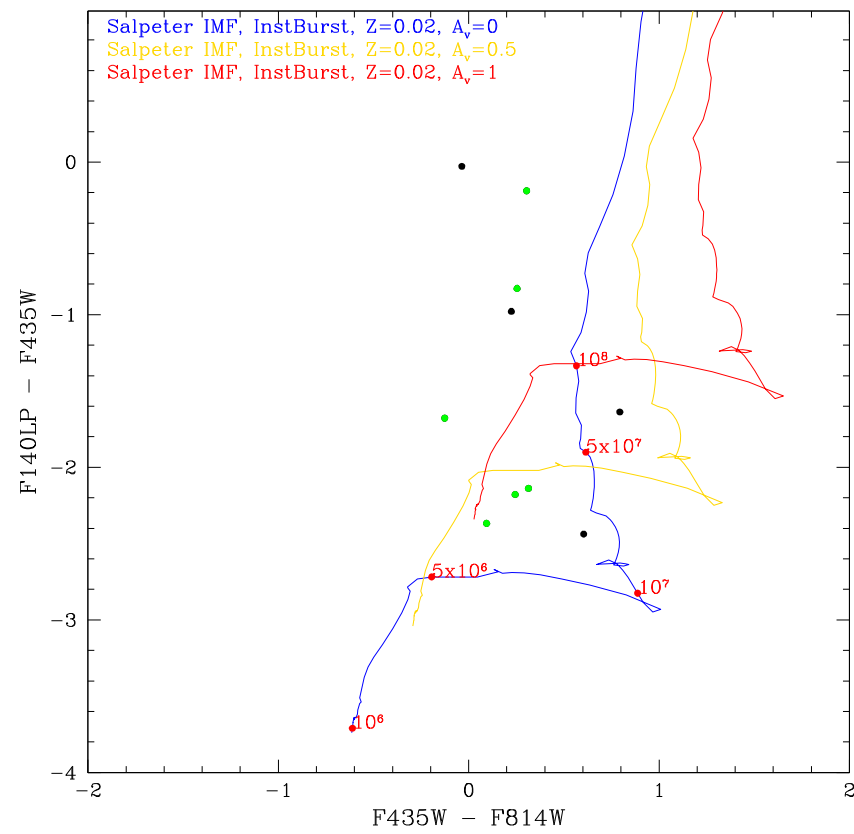

Figure 20. Color-color plot of all star clusters identified in 2MASX J06094582-2140234 in F814W, F435W, and F140LP plotted against SSP models with various amounts of visual extinction. The green points correspond to the clusters found in dustier regions of the galaxy in Figure 19.

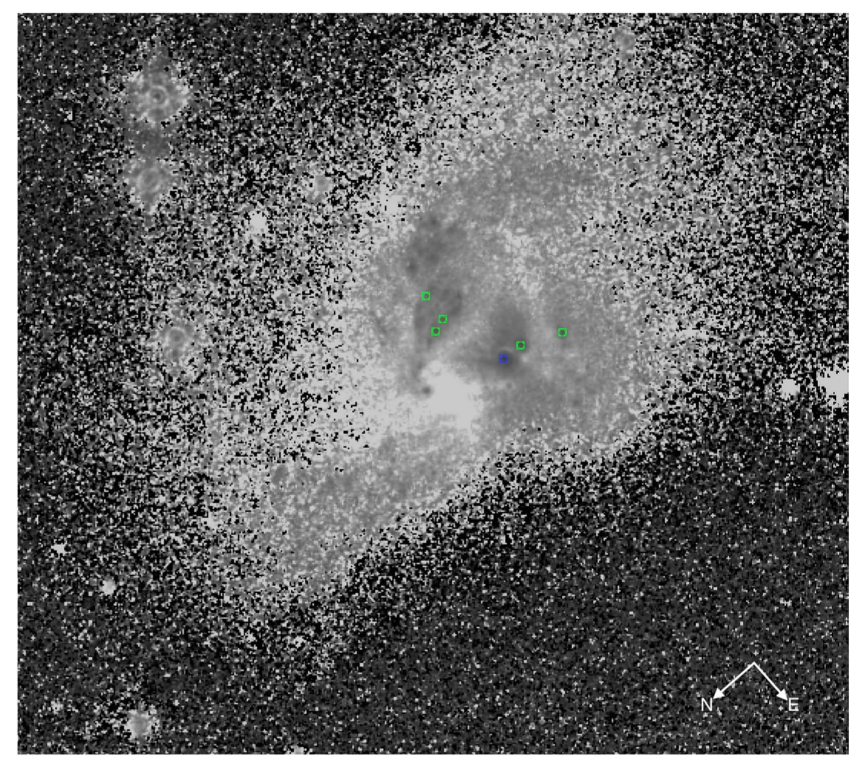

Figure 21. Inverted black and white $B-I$ image of 2MASX J08370182 4954302 taken with HST ACS/WFC F814W and F435W. The bright emission corresponds to redder (i.e., dustier) regions of the galaxy. The blue centroids correspond to clusters found in relatively "dust-free" regions of these galaxies, whereas the green centroids correspond to clusters found in relatively dustier regions of the galaxy.

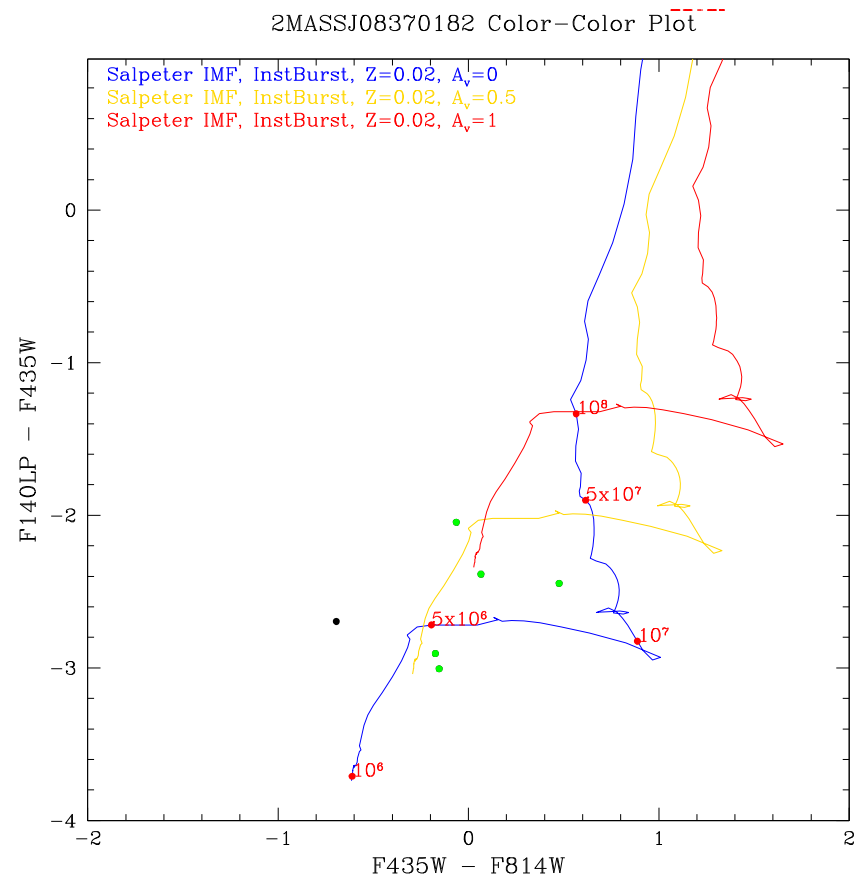

Figure 22. Color-color plot of all star clusters identified in 2MASX J08370182-4954302 in F814W, F435W, and F140LP plotted against SSP models with various amounts of visual extinction. The green points correspond to the clusters found in dustier regions of the galaxy in Figure 21. 


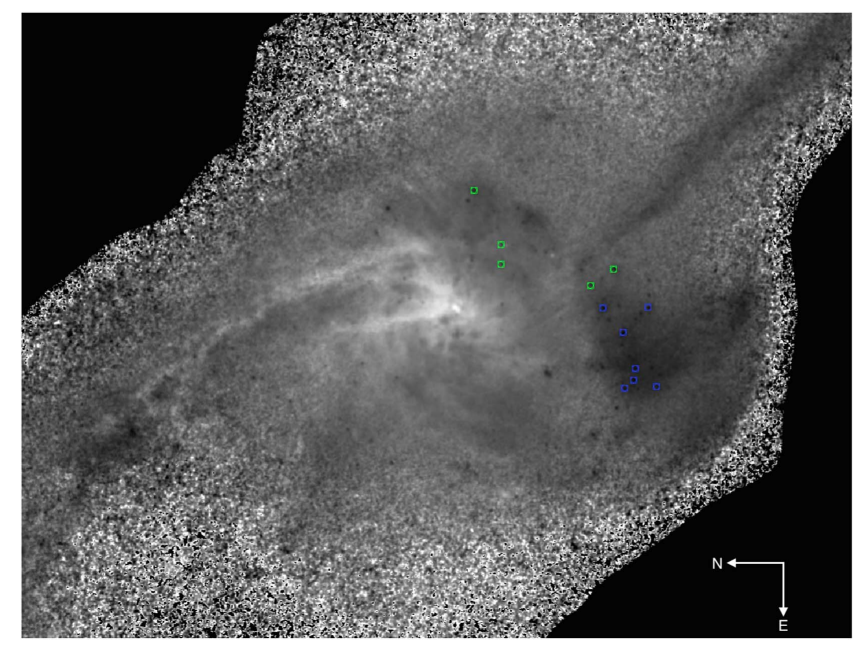

Figure 23. Inverted black and white $B-I$ image taken of NGC 2623 with $H S T$ ACS/WFC F814W and F435W. The bright emission corresponds to redder (i.e., dustier) regions of the galaxy. The blue centroids correspond to clusters found in relatively "dust-free" regions of these galaxies, whereas the green centroids correspond to clusters found in relatively dustier regions of the galaxy.

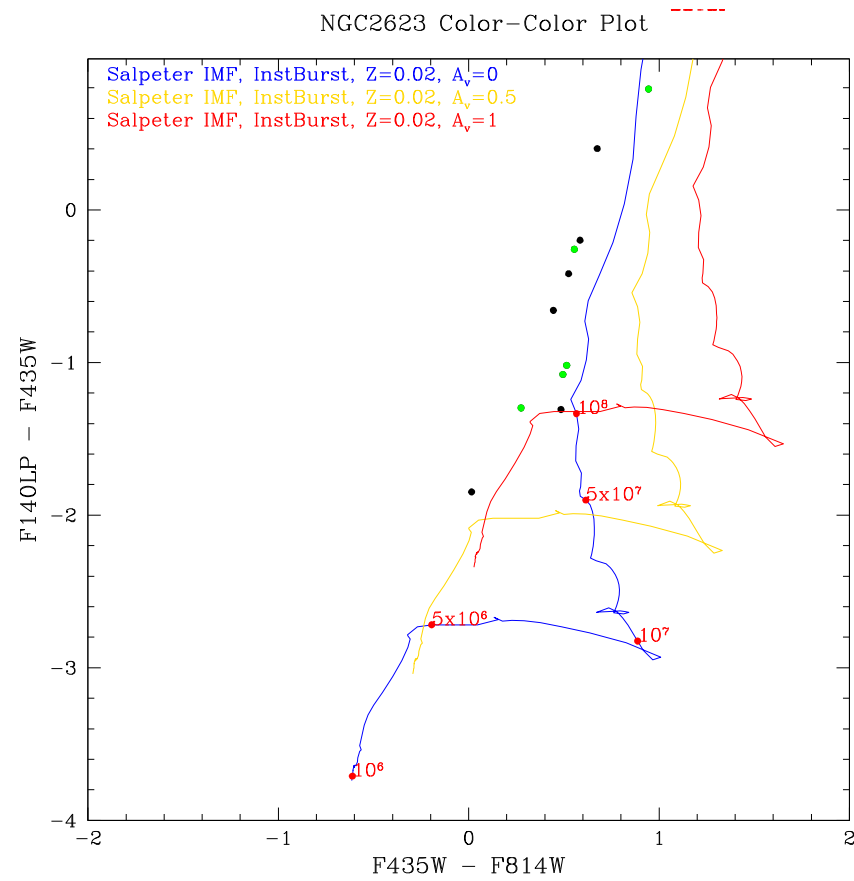

Figure 24. Color-color plot of all star clusters identified in NGC 2623 in F814W, F435W, and F140LP plotted against SSP models with various amounts of visual extinction. The green points correspond to the clusters found in dustier regions of the galaxy in Figure 23.

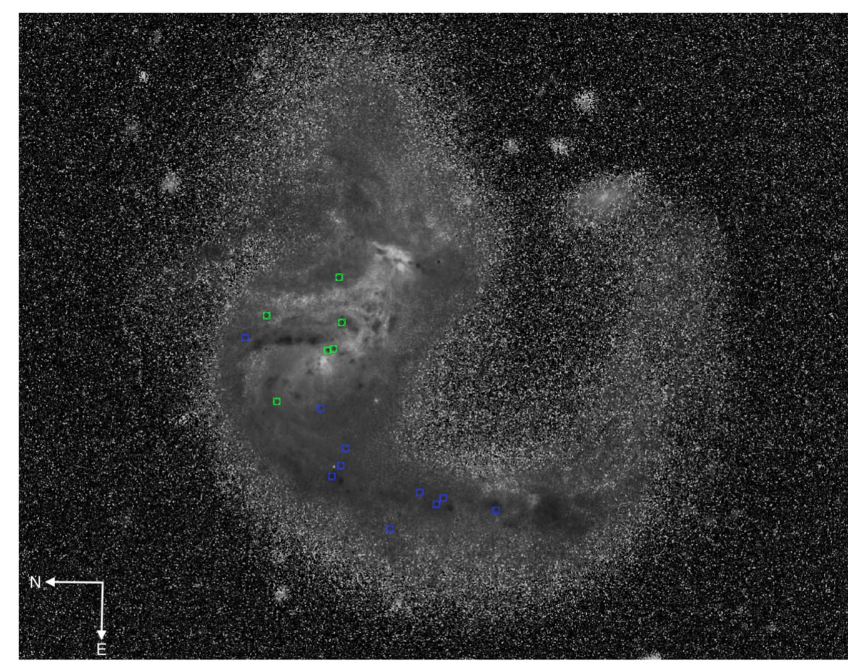

Figure 25. Inverted black and white $B-I$ image of UGC 04881 taken with $H S T$ ACS/WFC F814W and F435W. The bright emission corresponds to redder (i.e., dustier) regions of the galaxy. The blue centroids correspond to clusters found in relatively "dust-free" regions of these galaxies, whereas the green centroids correspond to clusters found in relatively dustier regions of the galaxy.

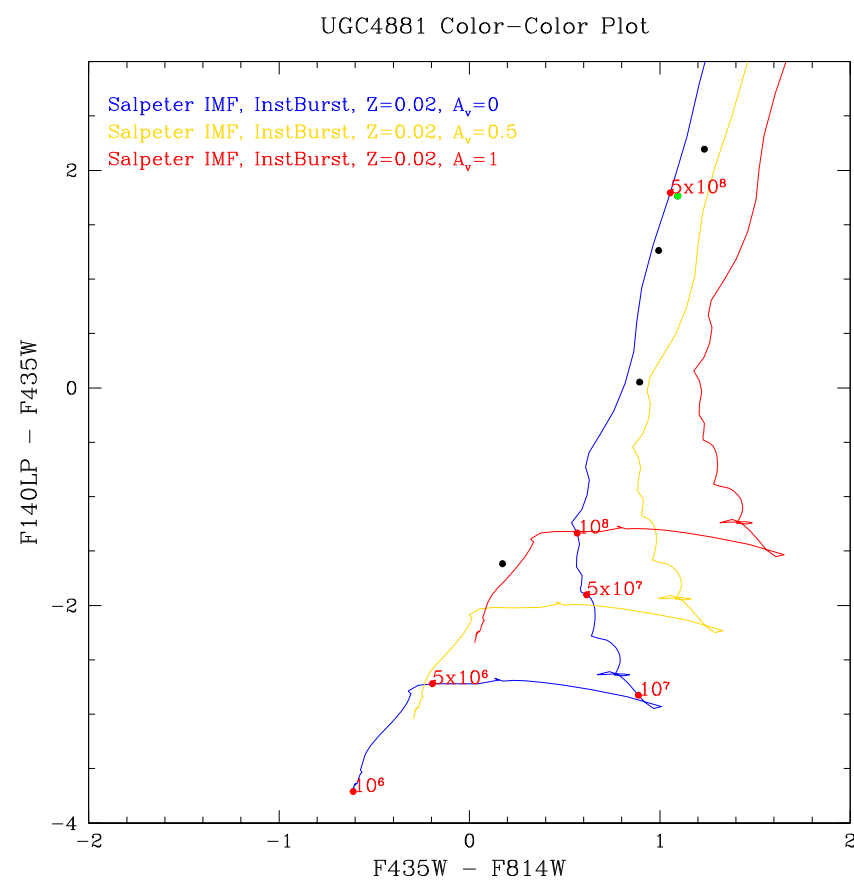

Figure 26. Color-color plot of all star clusters identified in UGC 04881 in F814W, F435W, and F140LP plotted against SSP models with various amounts of visual extinction. The green points correspond to the clusters found in dustier regions of the galaxy in Figure 25. 


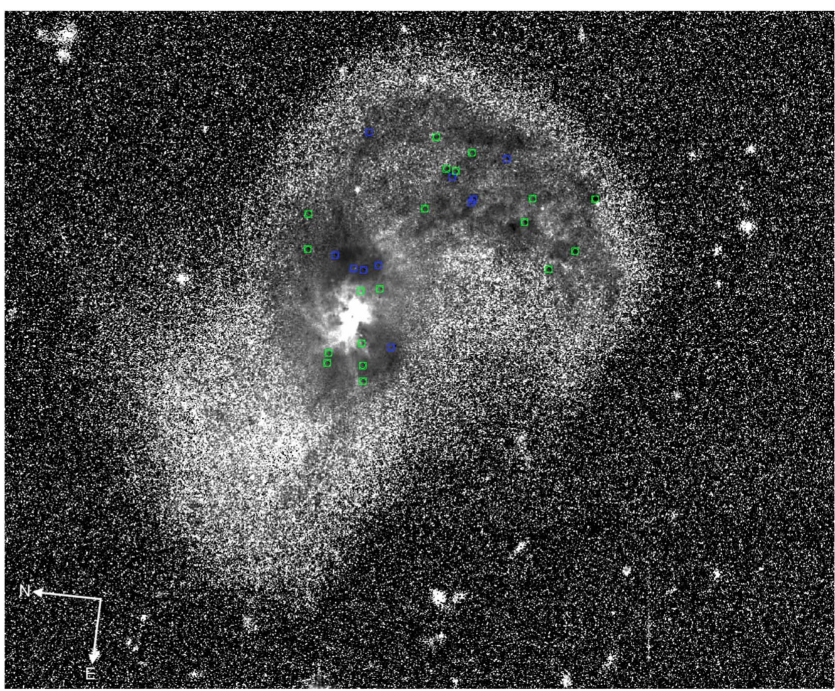

Figure 27. Inverted black and white $B-I$ image of IC 2545 taken with $H S T$ ACS/WFC F814W and F435W. The bright emission corresponds to redder (i.e., dustier) regions of the galaxy. The blue centroids correspond to clusters found in relatively "dust-free" regions of these galaxies, whereas the green centroids correspond to clusters found in relatively dustier regions of the galaxy.

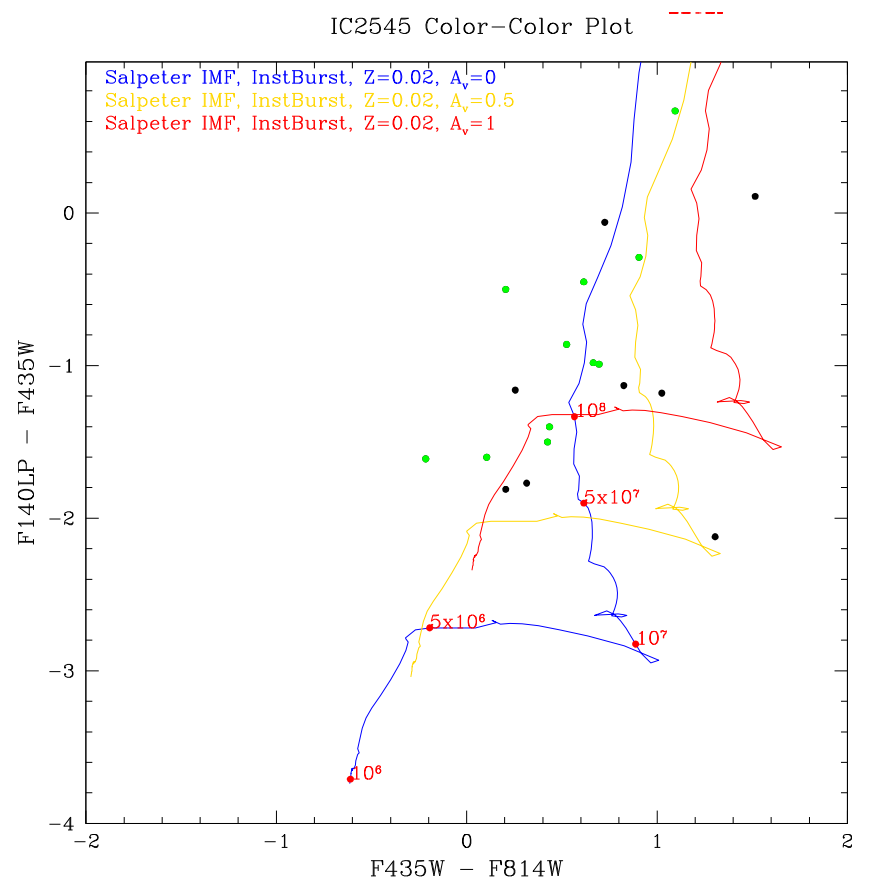

Figure 28. Color-color plot of all star clusters identified in IC 2545 in F814W, F435W, and F140LP plotted against SSP models with various amounts of visual extinction. The green points correspond to the clusters found in dustier regions of the galaxy in Figure 27.

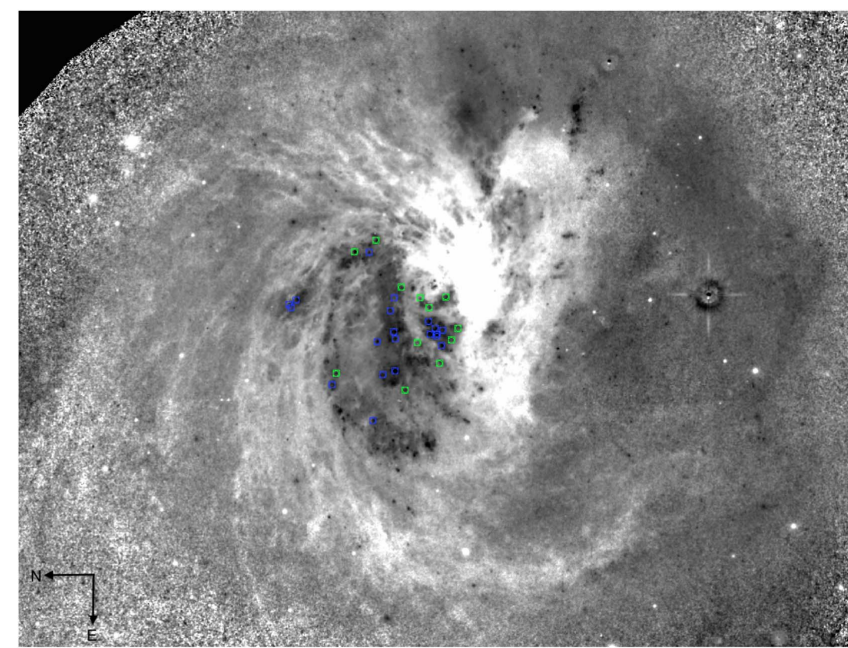

Figure 29. Inverted black and white $B-I$ image of NGC 3256 taken with $H S T$ ACS/WFC F814W and F435W. The bright emission corresponds to redder (i.e., dustier) regions of the galaxy. The blue centroids correspond to clusters found in relatively "dust-free" regions of these galaxies, whereas the green centroids correspond to clusters found in relatively dustier regions of the galaxy.

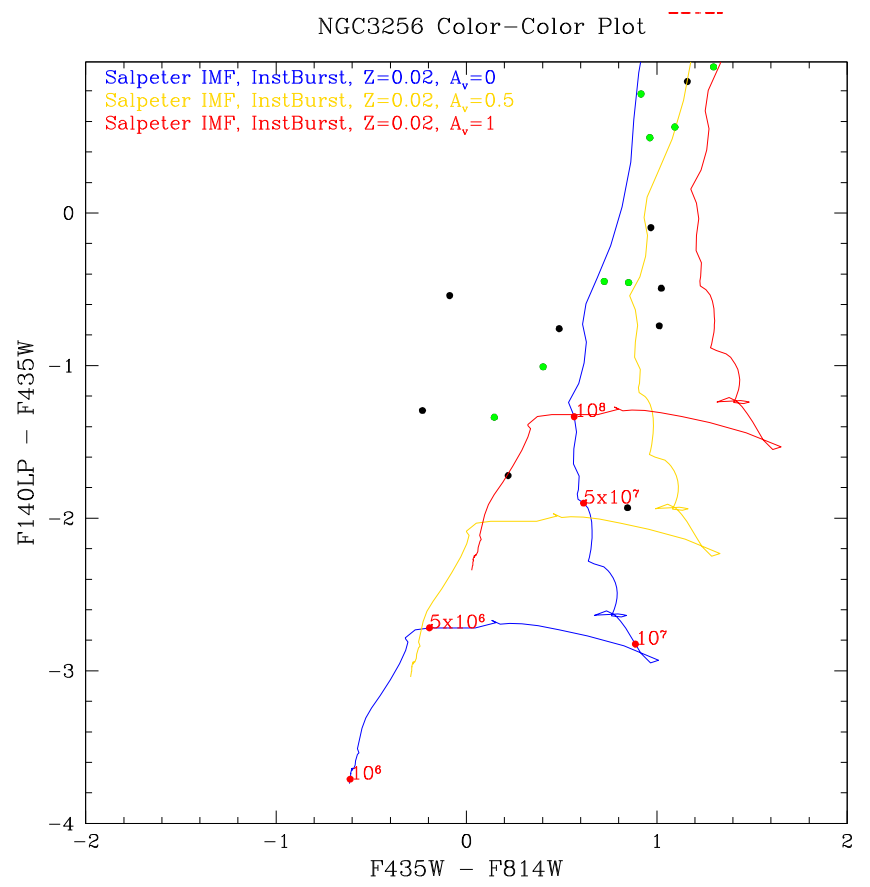

Figure 30. Color-color plot of all star clusters identified in NGC 3256 in F814W, F435W, and F140LP plotted against SSP models with various amounts of visual extinction. The green points correspond to the clusters found in dustier regions of the galaxy in Figure 29. 


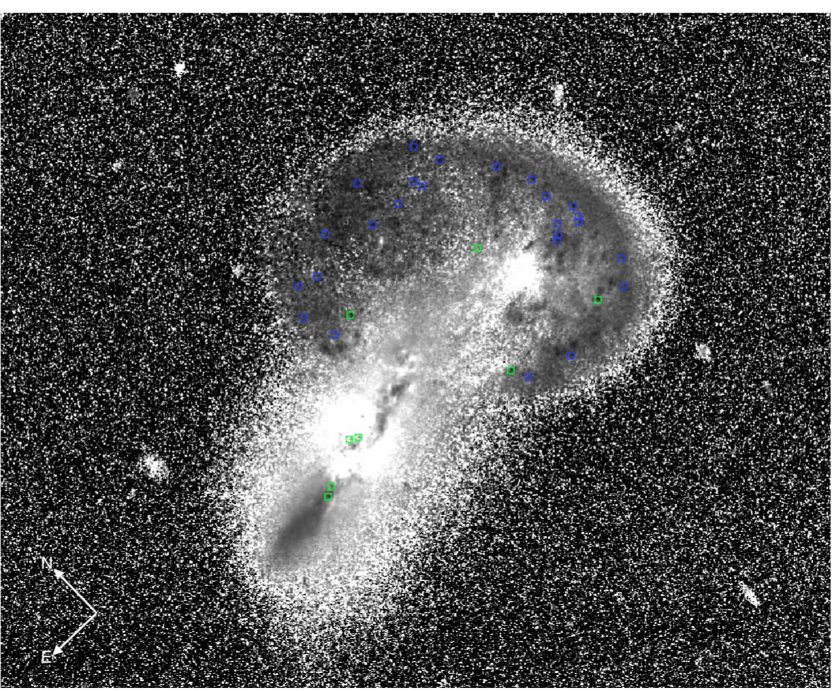

Figure 31. Inverted black and white $B-I$ image of Arp 148 taken with $H S T$ ACS/WFC F814W and F435W. The bright emission corresponds to redder (i.e., dustier) regions of the galaxy. The blue centroids correspond to clusters found in relatively "dust-free" regions of these galaxies, whereas the green centroids correspond to clusters found in relatively dustier regions of the galaxy.

ARP148 Color-Color Plot -----

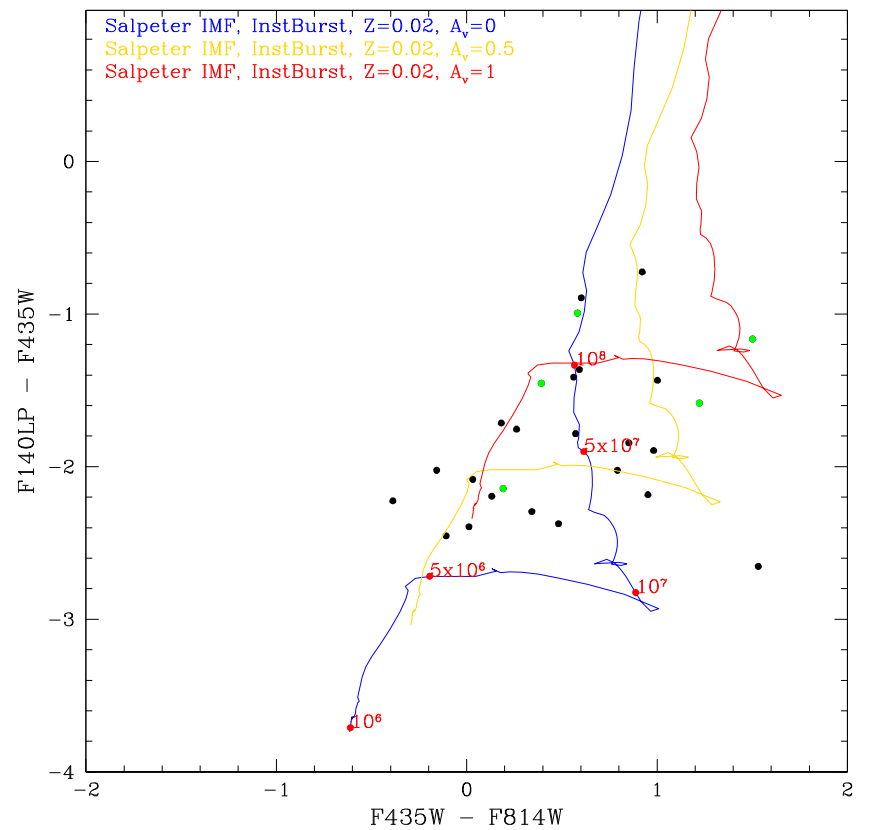

Figure 32. Color-color plot of all star clusters identified in Arp 148 in F814W, F435W, and F140LP plotted against SSP models with various amounts of visual extinction. The green points correspond to the clusters found in dustier regions of the galaxy in Figure 31.

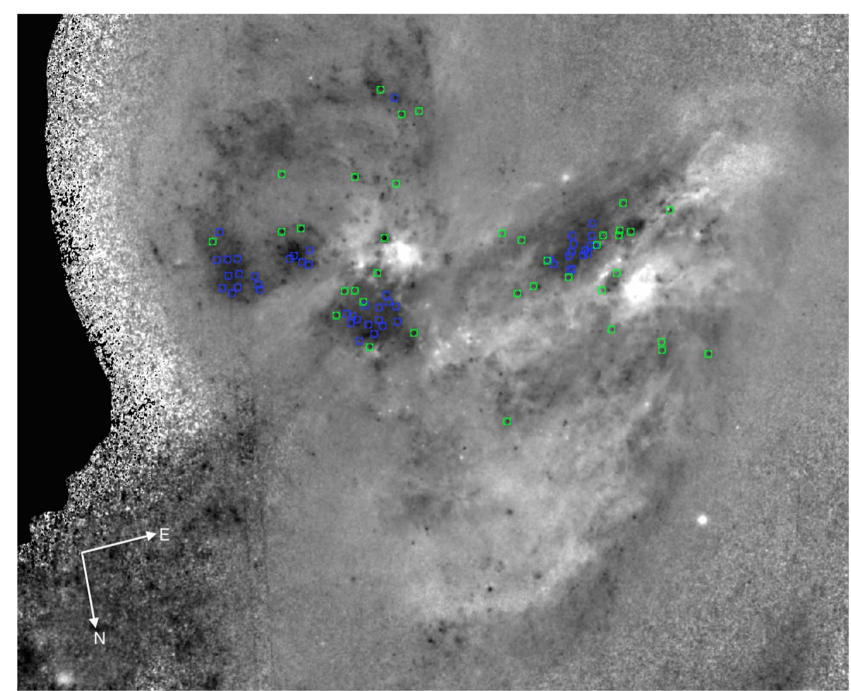

Figure 33. Inverted black and white $B-I$ image of NGC $3690 \mathrm{E} / \mathrm{W}$ taken with HST ACS/WFC F814W and F435W. The bright emission corresponds to redder (i.e., dustier) regions of the galaxy. The blue centroids correspond to clusters found in relatively "dust-free" regions of these galaxies, whereas the green centroids correspond to clusters found in relatively dustier regions of the galaxy.

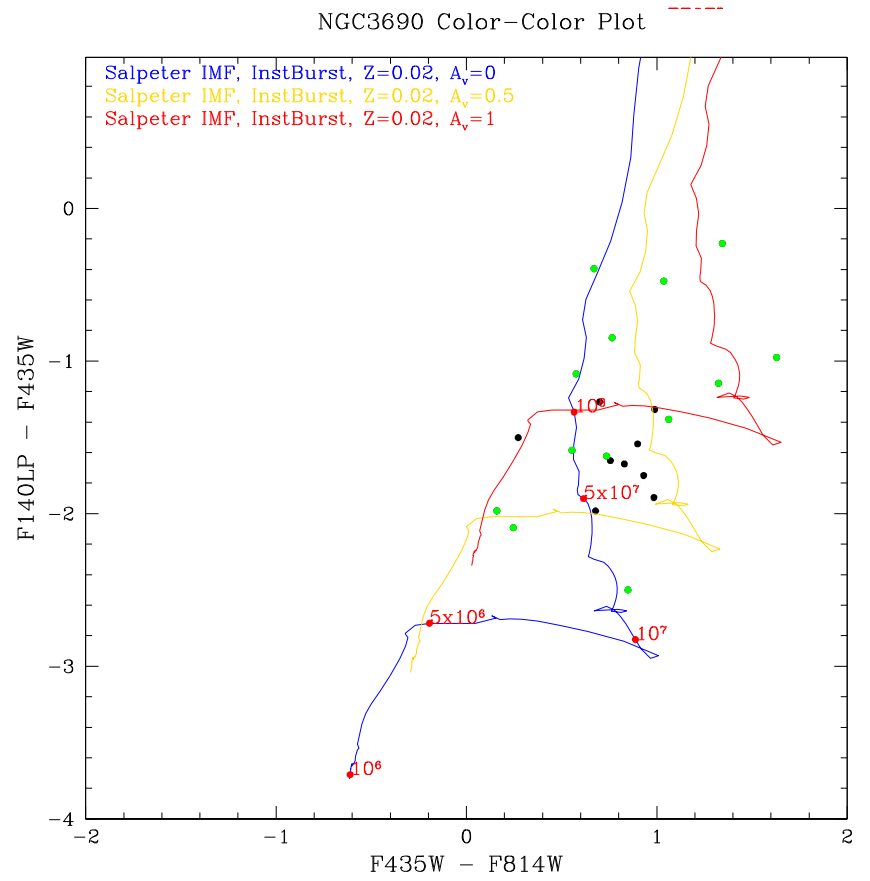

Figure 34. Color-color plot of all star clusters identified in NGC 3690E in F814W, F435W, and F140LP plotted against SSP models with various amounts of visual extinction. The green points correspond to the clusters found in dustier regions of the galaxy in Figure 33. 


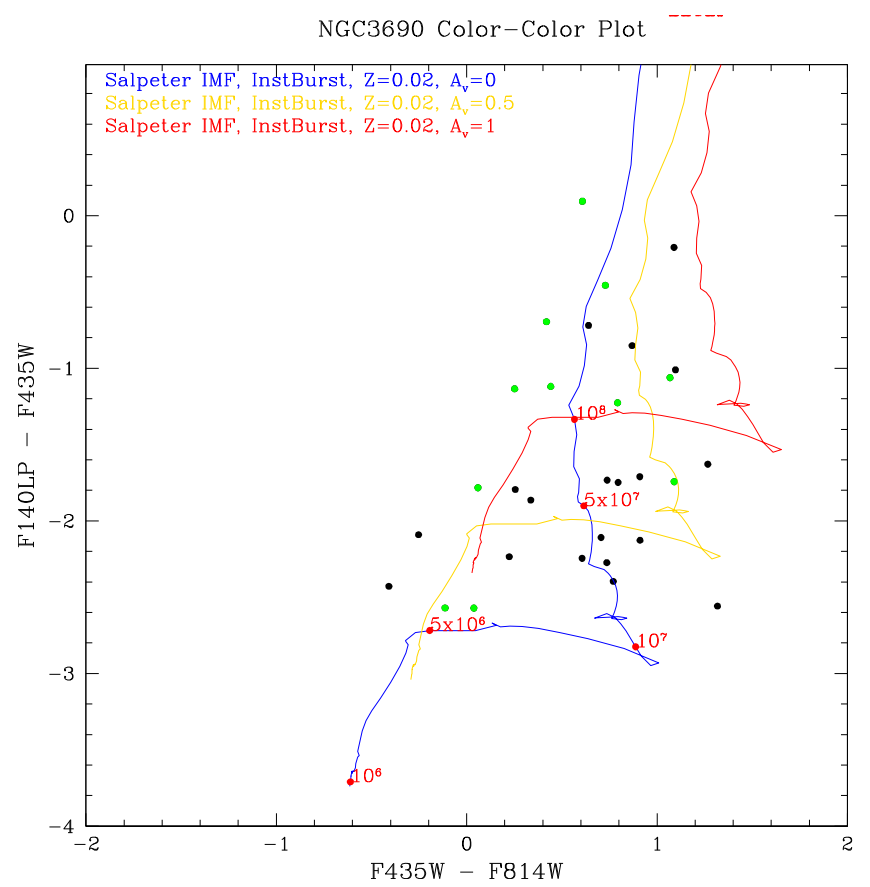

Figure 35. Color-color plot of all star clusters identified in NGC 3690W in F814W, F435W, and F140LP plotted against SSP models with various amounts of visual extinction. The green points correspond to the clusters found in dustier regions of the galaxy in Figure 33.

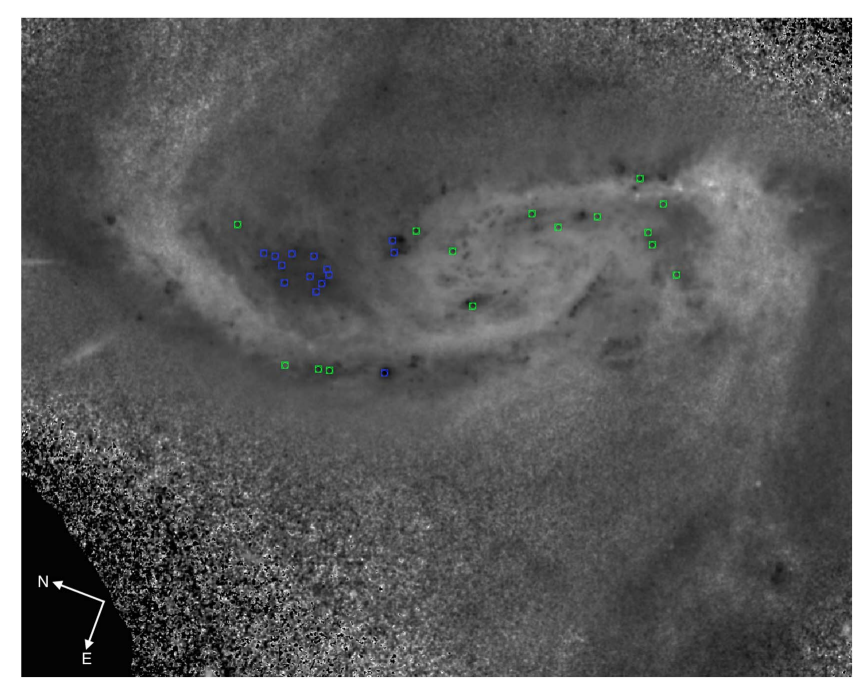

Figure 36. Inverted black and white $B-I$ image of NGC 5257E taken with $H S T$ ACS/WFC F814W and F435W. The bright emission corresponds to redder (i.e., dustier) regions of the galaxy. The blue centroids correspond to clusters found in relatively "dust-free" regions of these galaxies, whereas the green centroids correspond to clusters found in relatively dustier regions of the galaxy.

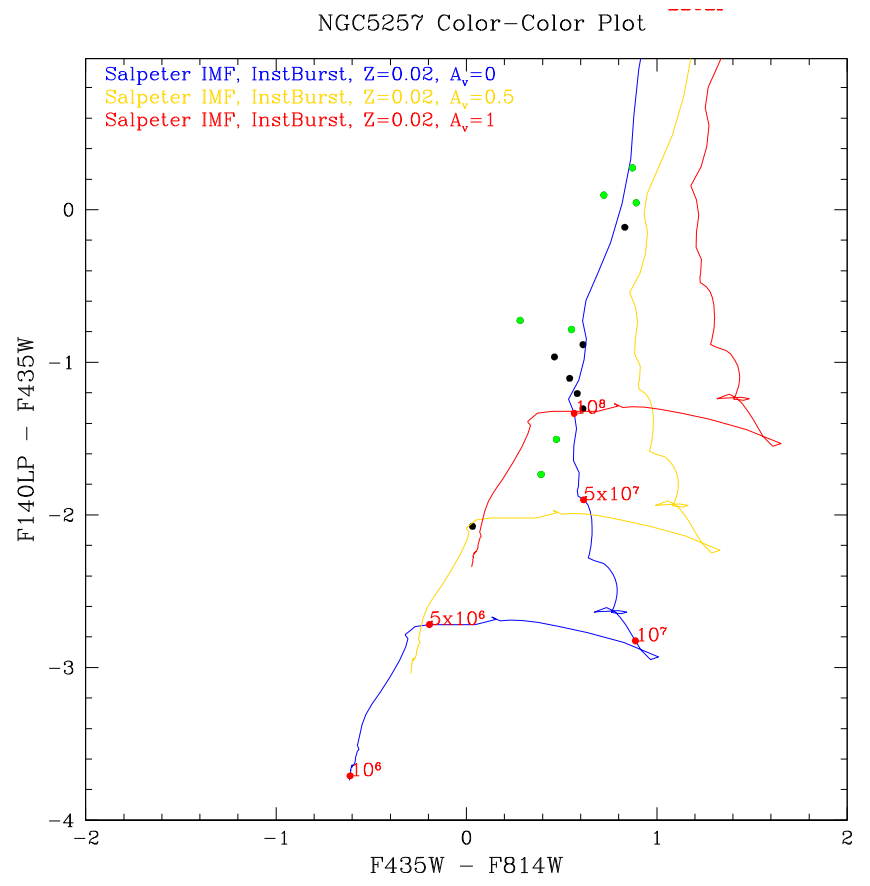

Figure 37. Color-color plot of all star clusters identified in NGC 5257E F814W, F435W, and F140LP plotted against SSP models with various amounts of visual extinction. The green points correspond to the clusters found in dustier regions of the galaxy in Figure 36.

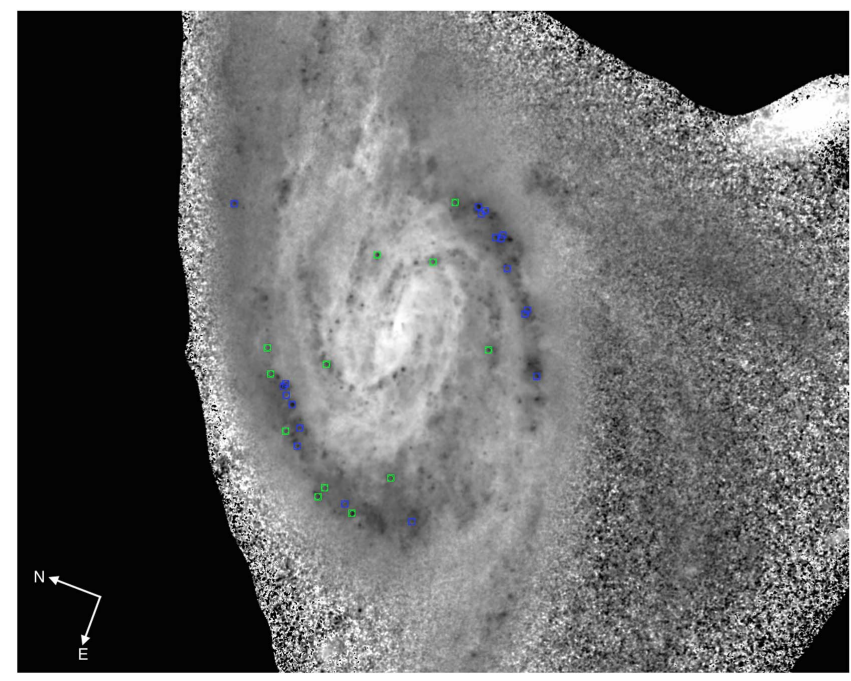

Figure 38. Inverted black and white $B-I$ image of NGC $5257 \mathrm{~W}$ taken with HST ACS/WFC F814W and F435W. The bright emission corresponds to redder (i.e., dustier) regions of the galaxy. The blue centroids correspond to clusters found in relatively "dust-free" regions of these galaxies, whereas the green centroids correspond to clusters found in relatively dustier regions of the galaxy. 


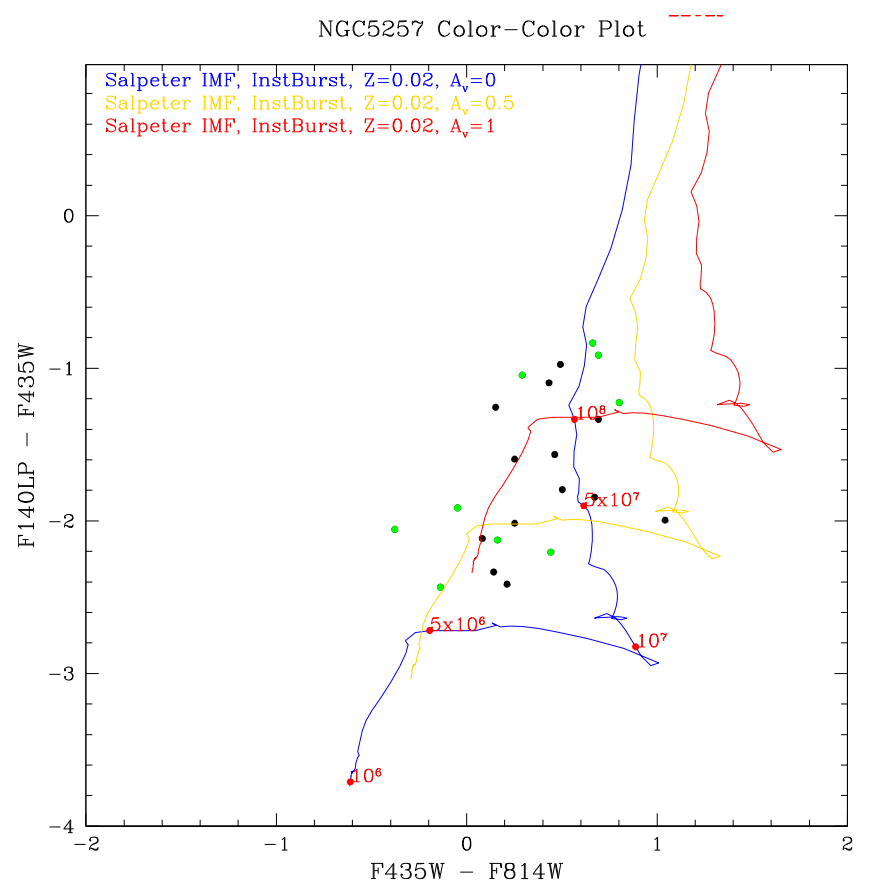

Figure 39. Color-color plot of all star clusters identified in NGC 5257W in F814W, F435W, and F140LP plotted against SSP models with various amounts of visual extinction. The green points correspond to the clusters found in dustier regions of the galaxy in Figure 38.

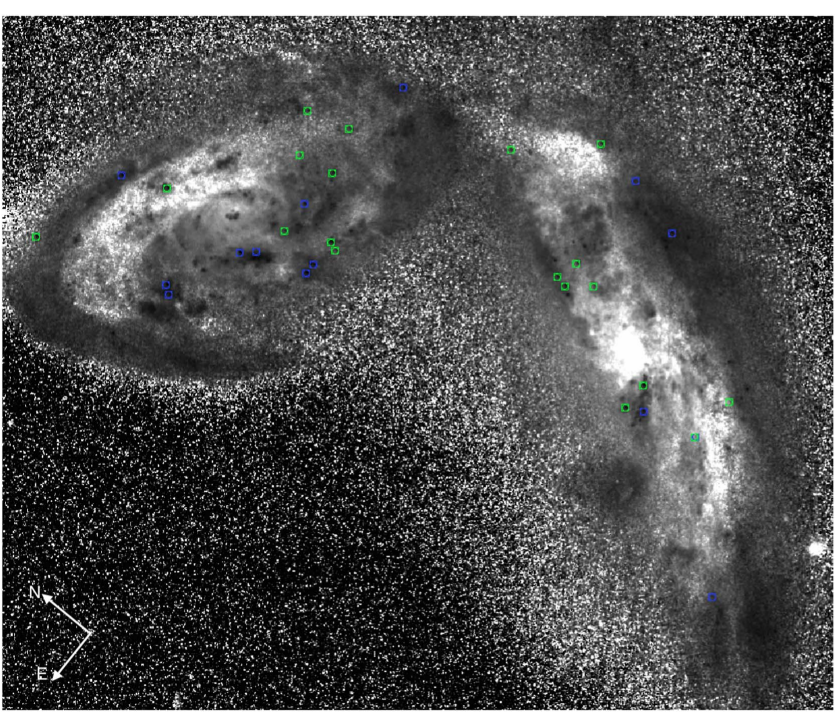

Figure 40. Inverted black and white $B-I$ image of NGC $5331 \mathrm{~N} / \mathrm{S}$ taken with HST ACS/WFC F814W and F435W. The bright emission corresponds to redder (i.e., dustier) regions of the galaxy. The blue centroids correspond to clusters found in relatively "dust-free" regions of these galaxies, whereas the green centroids correspond to clusters found in relatively dustier regions of the galaxy.

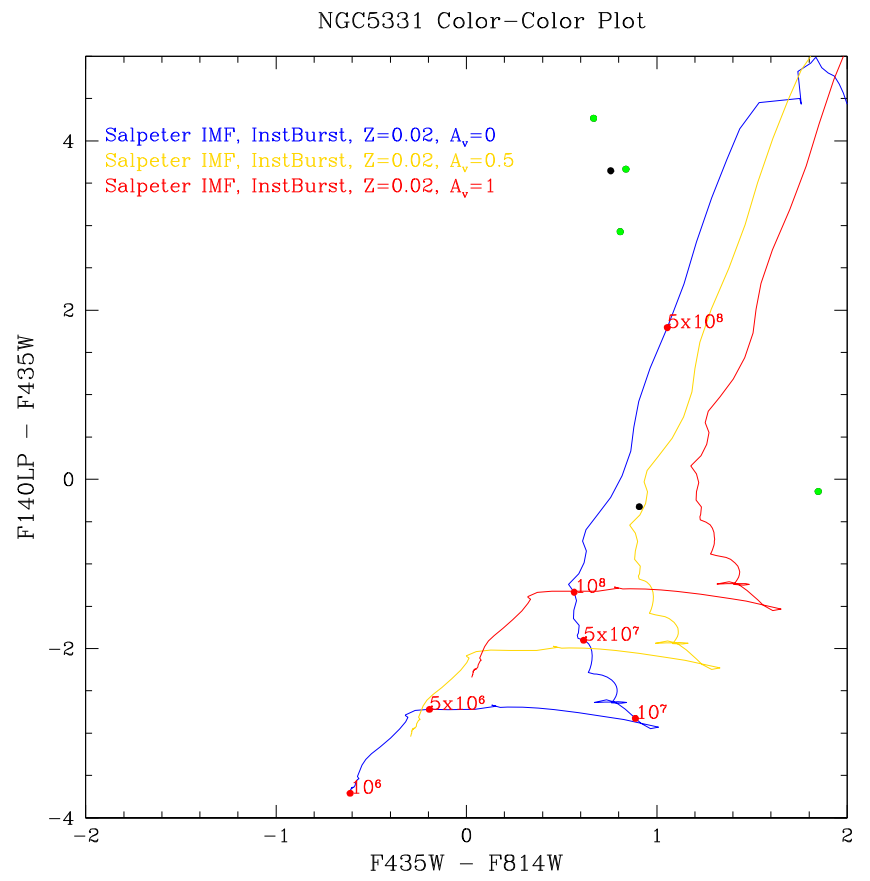

Figure 41. Color-color plot of all star clusters identified in NGC 5331S in F814W, F435W, and F140LP plotted against SSP models with various amounts of visual extinction. The green points correspond to the clusters found in dustier regions of the galaxy in Figure 40.

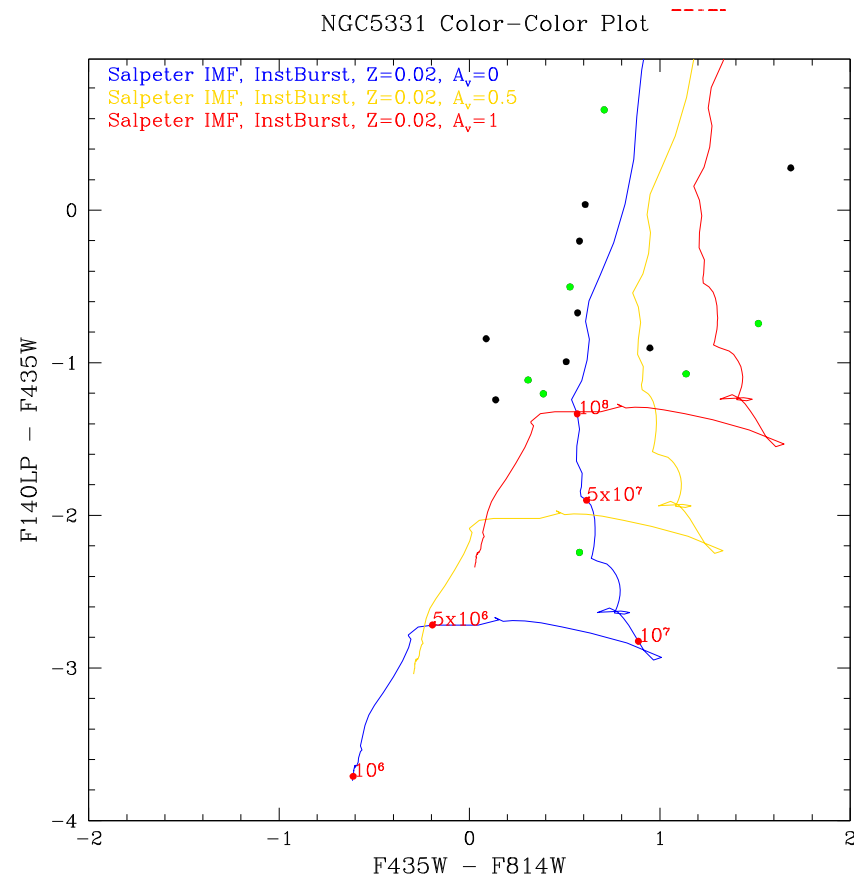

Figure 42. Color-color plot of all star clusters identified in NGC 5331N in F814W, F435W, and F140LP plotted against SSP models with various amounts of visual extinction. The green points correspond to the clusters found in dustier regions of the galaxy in Figure 40. 


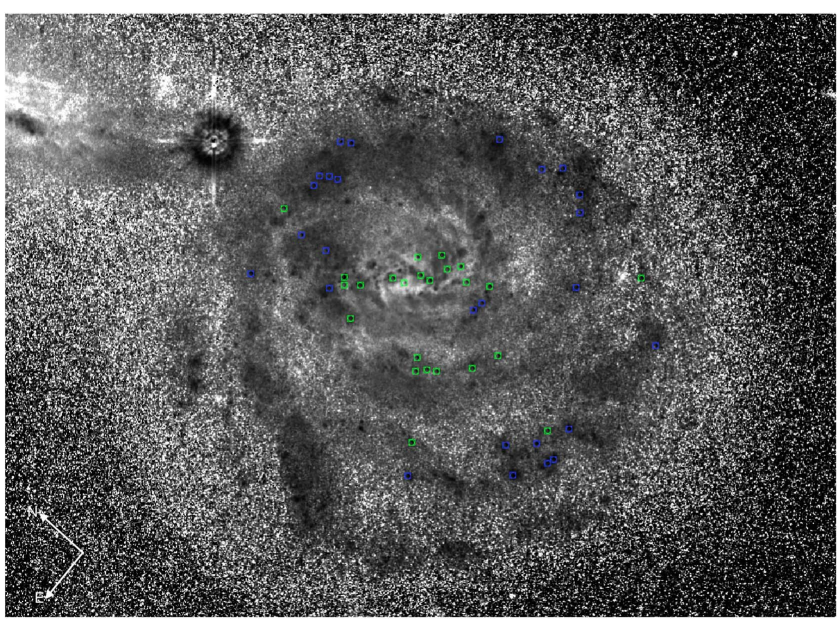

Figure 43. Inverted black and white $B-I$ image of UGC $09618 N E D 02$ taken with HST ACS/WFC F814W and F435W. The bright emission corresponds to redder (i.e., dustier) regions of the galaxy. The blue centroids correspond to clusters found in relatively "dust-free" regions of these galaxies, whereas the green centroids correspond to clusters found in relatively dustier regions of the galaxy.

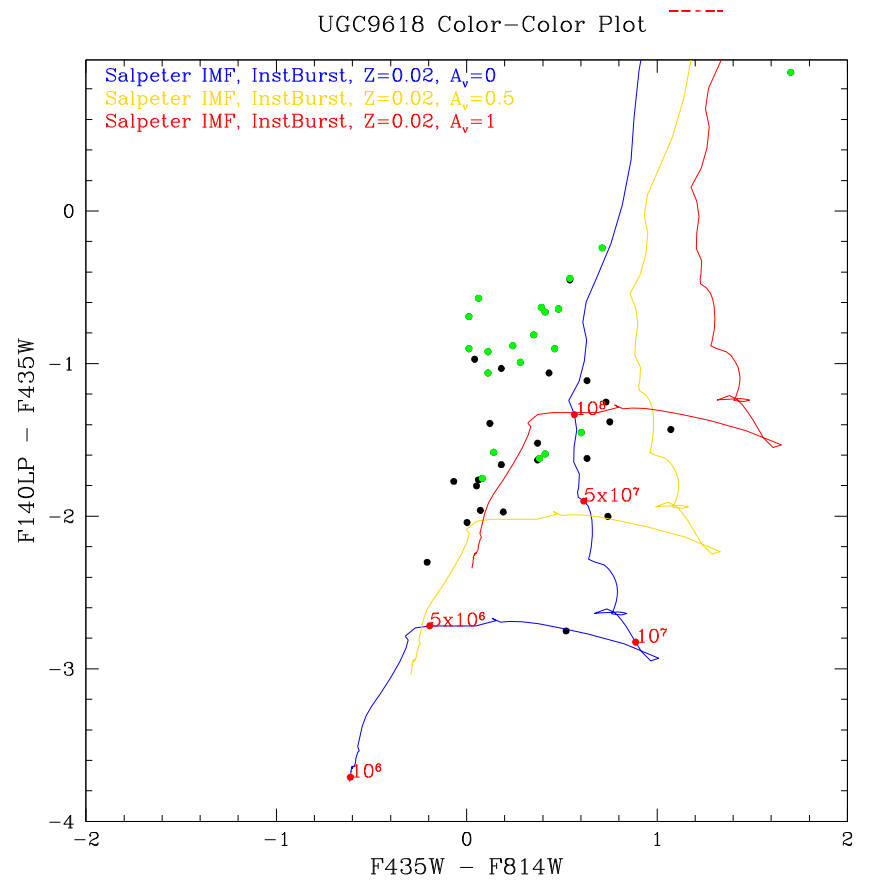

Figure 44. Color-color plot of all star clusters identified in UGC 09618NED02 in F814W, F435W, and F140LP plotted against SSP models with various amounts of visual extinction. The green points correspond to the clusters found in dustier regions of the galaxy in Figure 43.

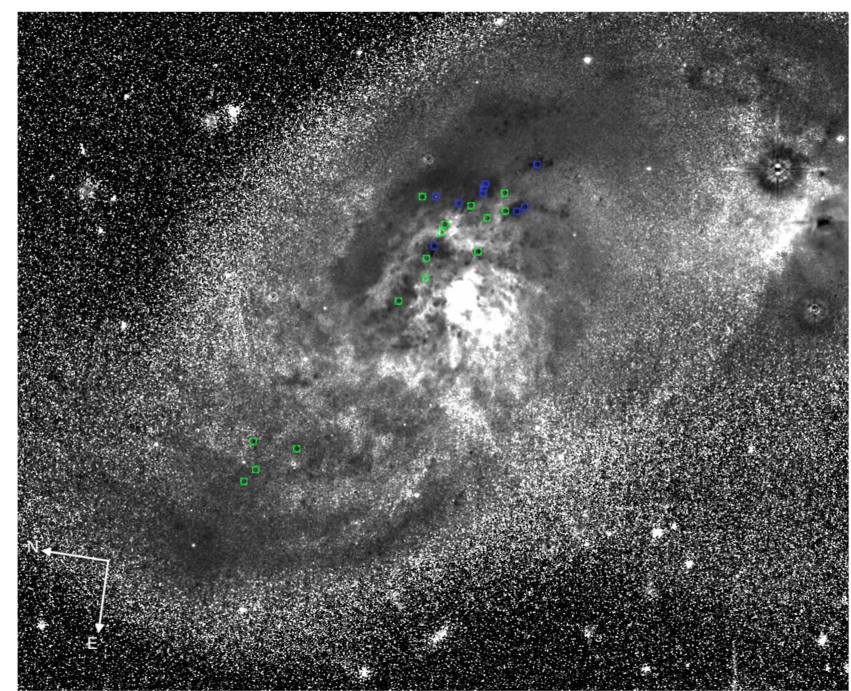

Figure 45. Inverted black and white $B-I$ image of IC $4687 \mathrm{~N}$ taken with $H S T$ ACS/WFC F814W and F435W. The bright emission corresponds to redder (i.e., dustier) regions of the galaxy. The blue centroids correspond to clusters found in relatively "dust-free" regions of these galaxies, whereas the green centroids correspond to clusters found in relatively dustier regions of the galaxy.

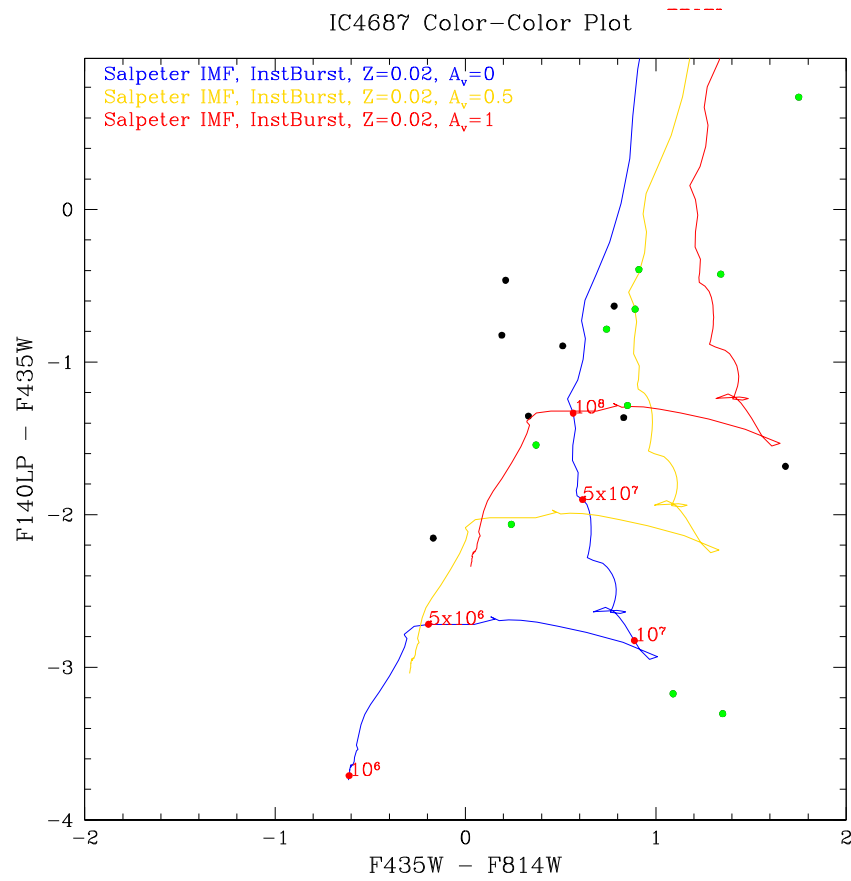

Figure 46. Color-color plot of all star clusters identified in IC $4687 \mathrm{~N}$ in F814W, F435W, and F140LP plotted against SSP models with various amounts of visual extinction. The green points correspond to the clusters found in dustier regions of the galaxy in Figure 45. 


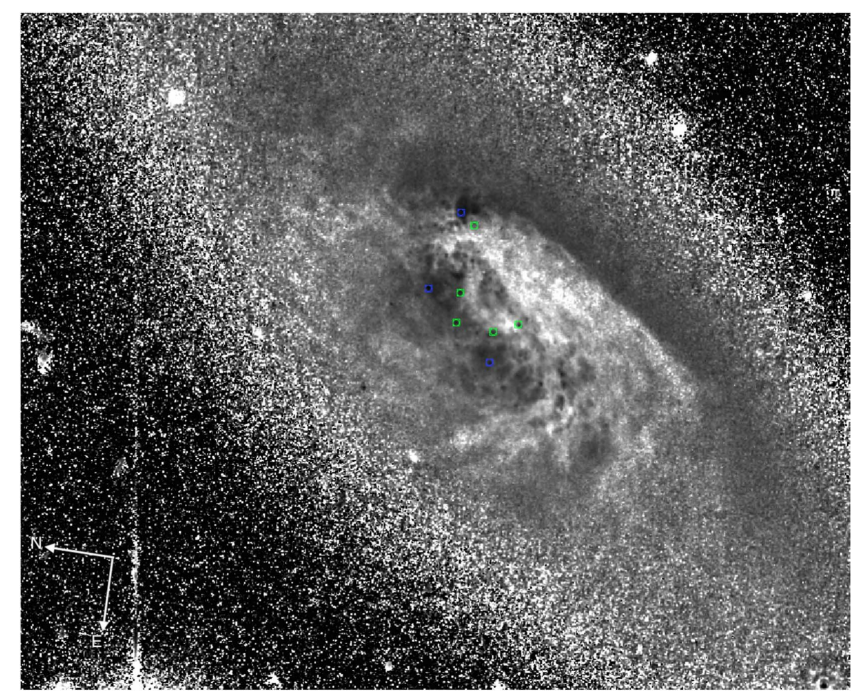

Figure 47. Inverted black and white $B-I$ image of IC $4687 \mathrm{~S}$ taken with $H S T$ ACS/WFC F814W and F435W. The bright emission corresponds to redder (i.e., dustier) regions of the galaxy. The blue centroids correspond to clusters found in relatively "dust-free" regions of these galaxies, whereas the green centroids correspond to clusters found in relatively dustier regions of the galaxy.

IC4687 Color-Color Plot

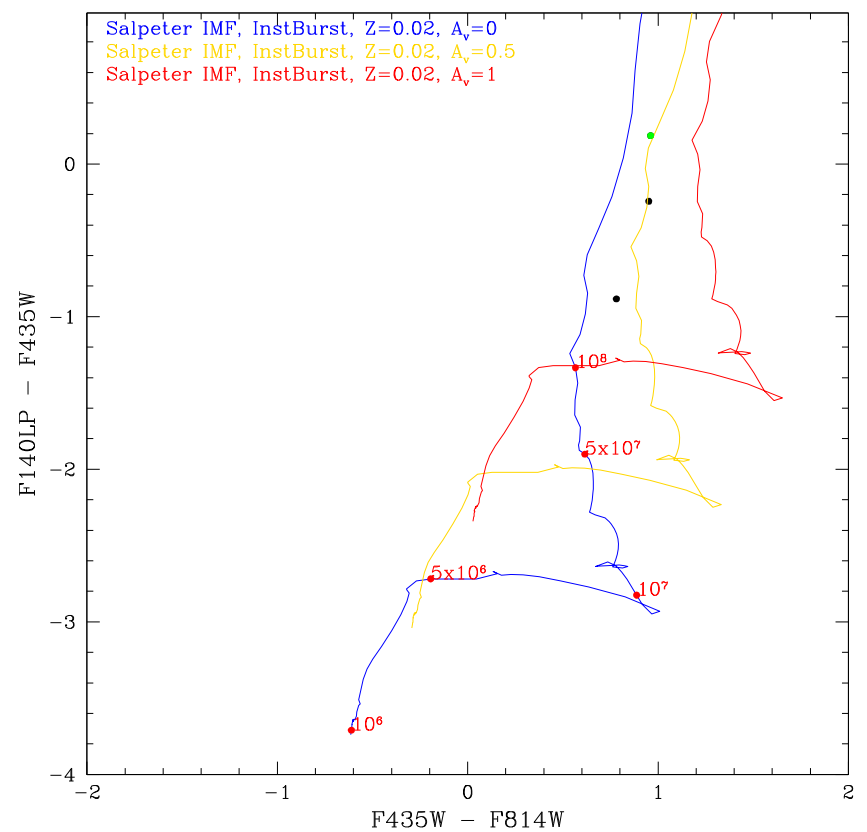

Figure 48. Color-color plot of all star clusters identified in IC 4687S in F814W, F435W, and F140LP plotted against SSP models with various amounts of visual extinction. The green points correspond to the clusters found in dustier regions of the galaxy in Figure 47.

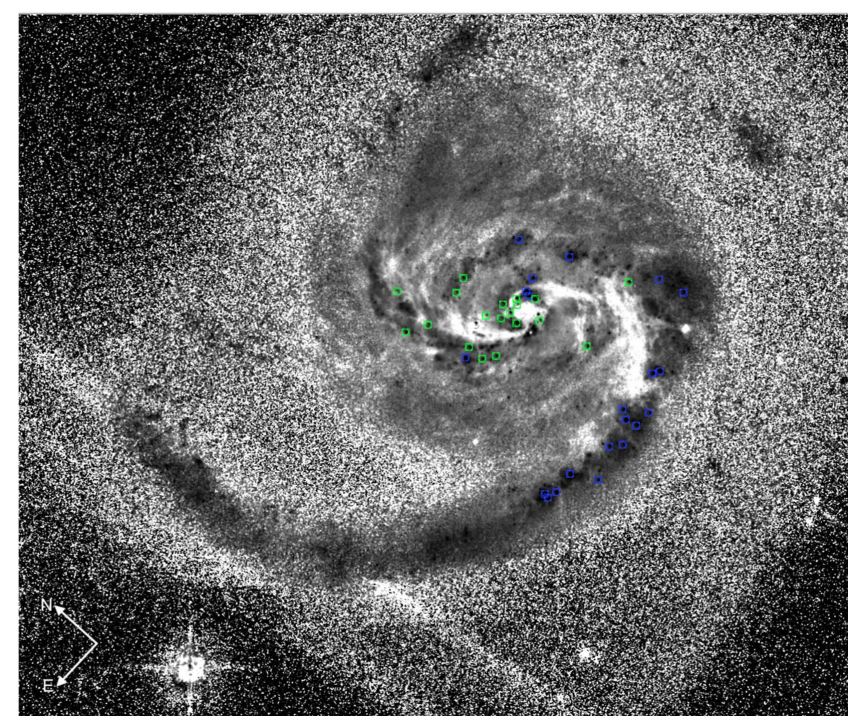

Figure 49. Inverted black and white $B-I$ image of NGC 6786 taken with $H S T$ ACS/WFC F814W and F435W. The bright emission corresponds to redder (i.e., dustier) regions of the galaxy. The blue centroids correspond to clusters found in relatively "dust-free" regions of these galaxies, whereas the green centroids correspond to clusters found in relatively dustier regions of the galaxy.

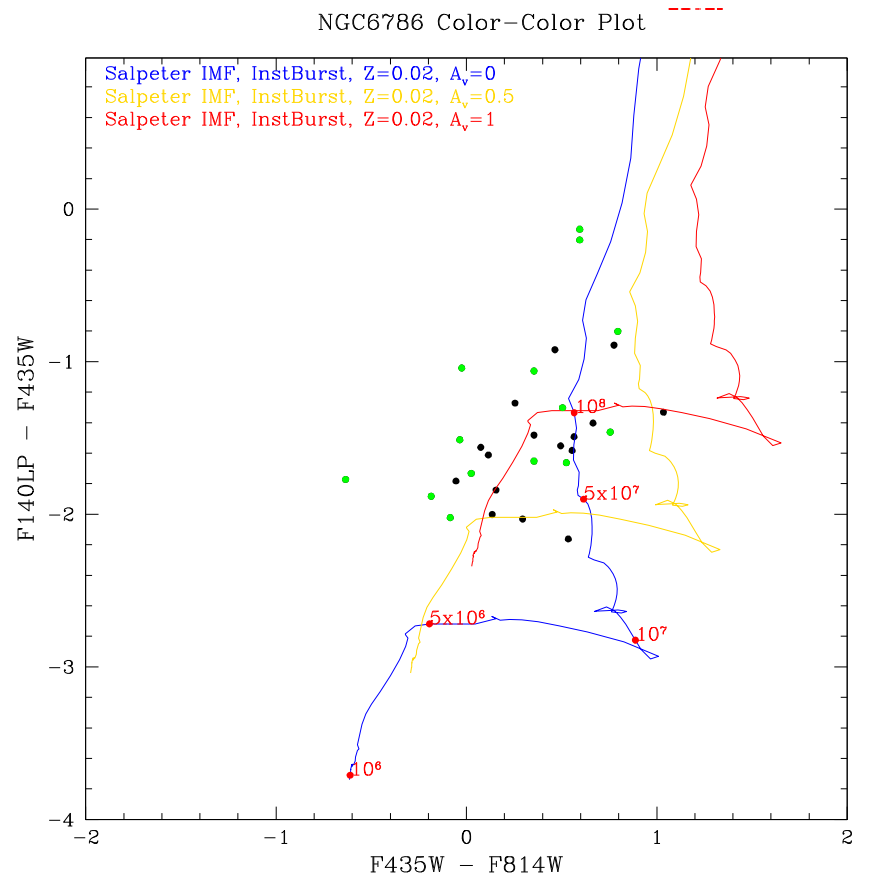

Figure 50. Color-color plot of all star clusters identified in NGC 6786 in F814W, F435W, and F140LP plotted against SSP models with various amounts of visual extinction. The green points correspond to the clusters found in dustier regions of the galaxy in Figure 49. 


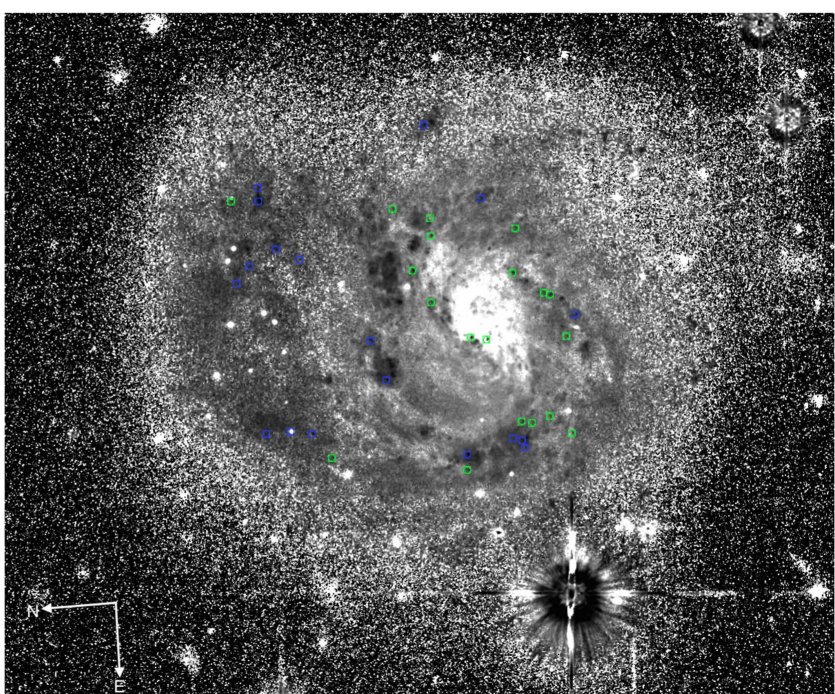

Figure 51. Inverted black and white $B-I$ image of IRAS $20351+2521$ taken with HST ACS /WFC F814W and F435W. The bright emission corresponds to redder (i.e., dustier) regions of the galaxy. The blue centroids correspond to clusters found in relatively "dust-free" regions of these galaxies, whereas the green centroids correspond to clusters found in relatively dustier regions of the galaxy.

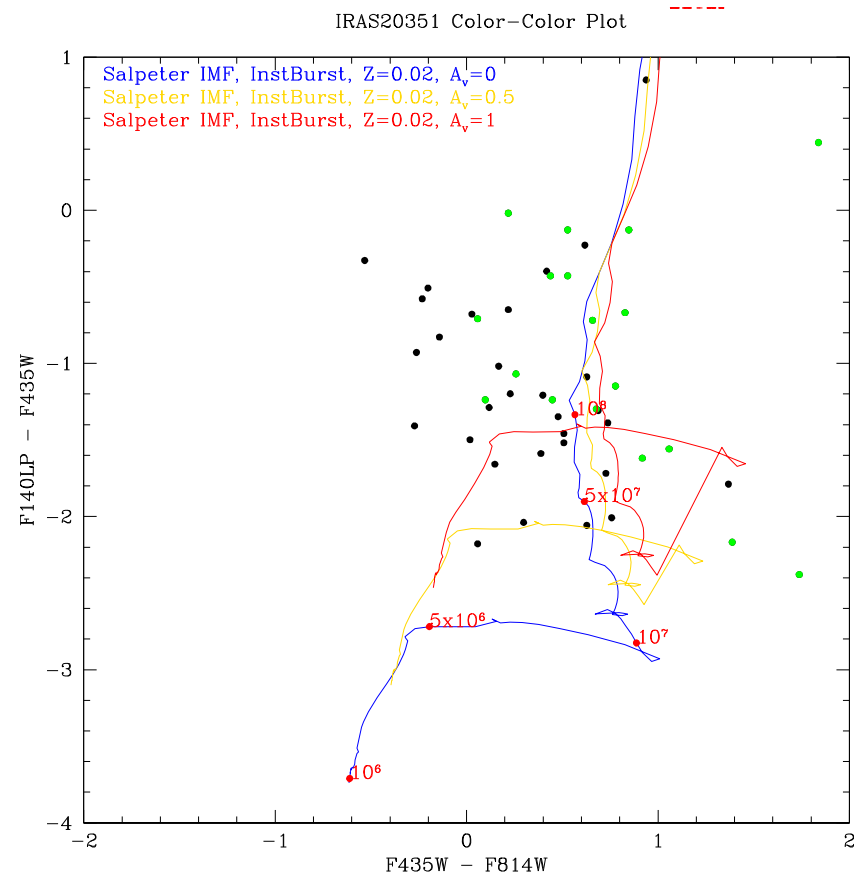

Figure 52. Color-color plot of all star clusters identified in IRAS 20351+2521 in F814W, F435W, and F140LP plotted against SSP models with various amounts of visual extinction. The green points correspond to the clusters found in dustier regions of the galaxy in Figure 51.

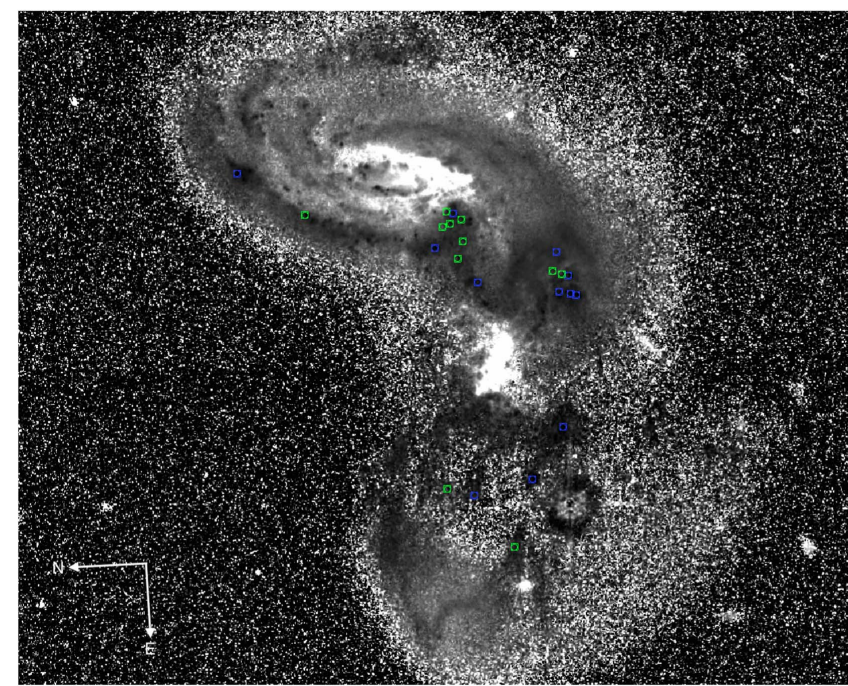

Figure 53. Inverted black and white $B-I$ image of II Zw 096 taken with $H S T$ ACS/WFC F814W and F435W. The bright emission corresponds to redder (i.e., dustier) regions of the galaxy. The blue centroids correspond to clusters found in relatively "dust-free" regions of these galaxies, whereas the green centroids correspond to clusters found in relatively dustier regions of the galaxy.

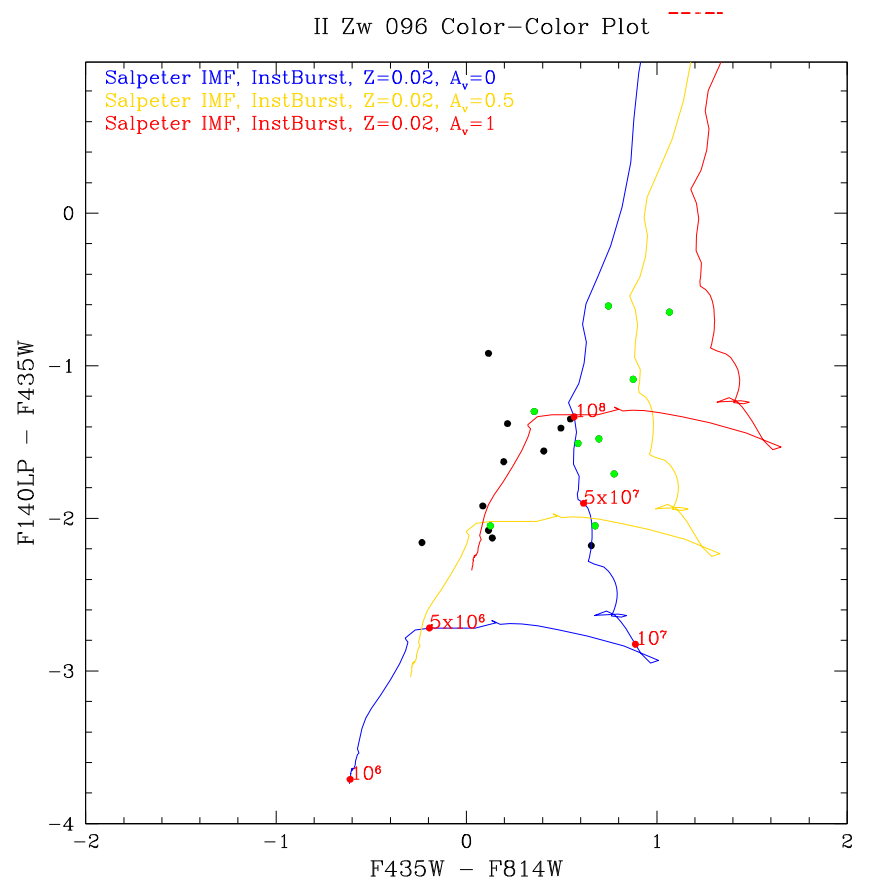

Figure 54. Color-color plot of all star clusters identified in II Zw 096 in F814W, F435W, and F140LP plotted against SSP models with various amounts of visual extinction. The green points correspond to the clusters found in dustier regions of the galaxy in Figure 53. 


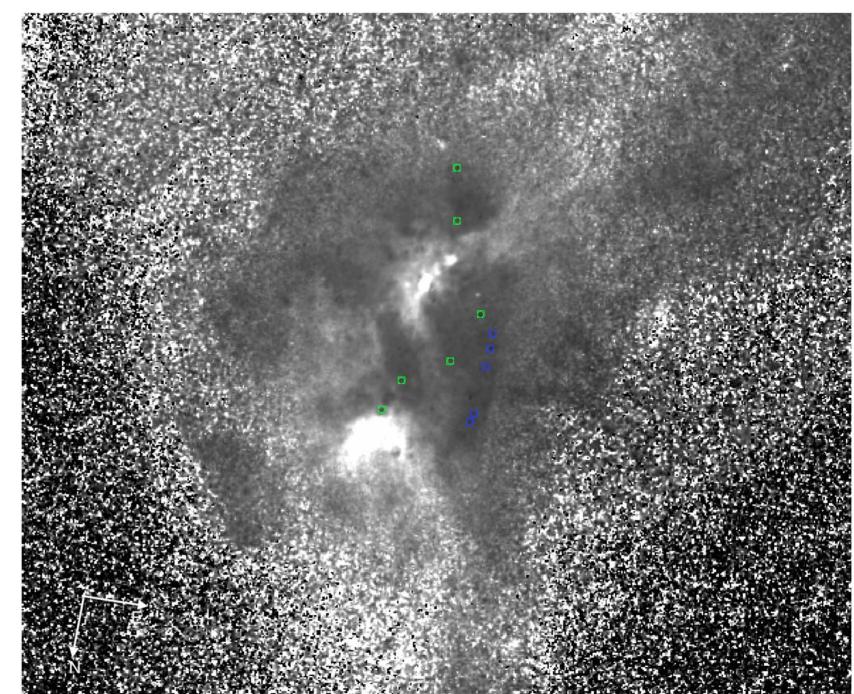

Figure 55. Inverted black and white $B-I$ image of ESO 148-IG002 taken with HST ACS/WFC F814W and F435W. The bright emission corresponds to redder (i.e., dustier) regions of the galaxy. The blue centroids correspond to clusters found in relatively "dust-free" regions of these galaxies, whereas the green centroids correspond to clusters found in relatively dustier regions of the galaxy.

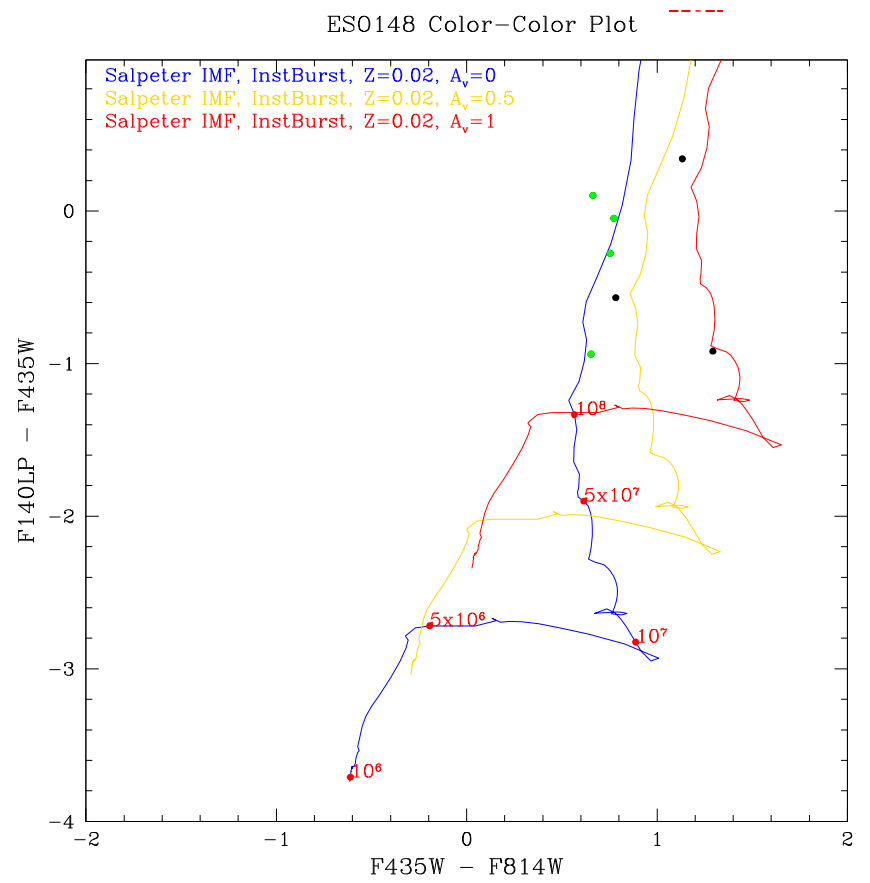

Figure 56. Color-color plot of all star clusters identified in ESO 148-IG002 in F814W, F435W, and F140LP plotted against SSP models with various amounts of visual extinction. The green points correspond to the clusters found in dustier regions of the galaxy in Figure 55.

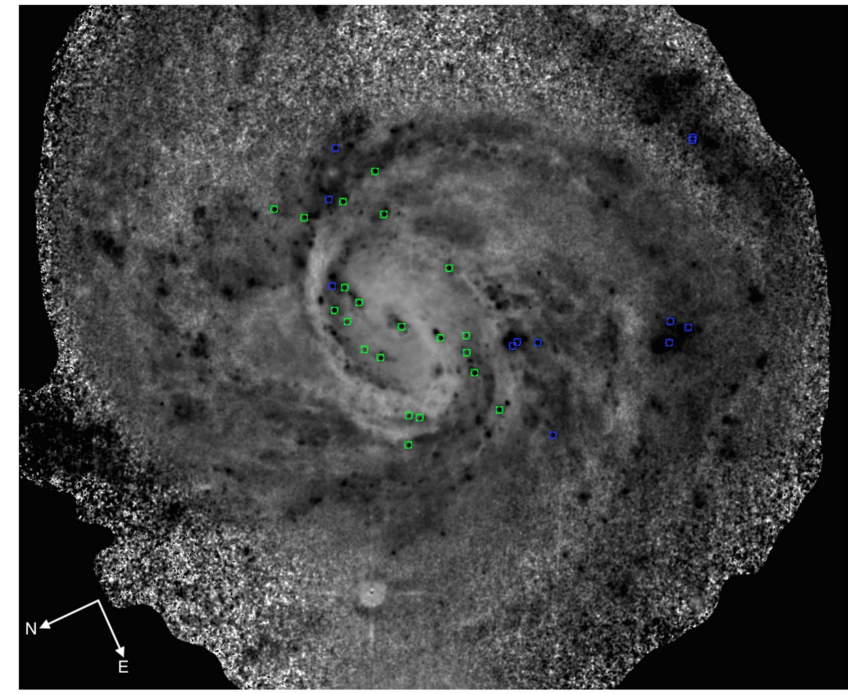

Figure 57. Inverted black and white $B-I$ image of NGC 7674 taken with $H S T$ ACS/WFC F814W and F435W. The bright emission corresponds to redder (i.e., dustier) regions of the galaxy. The blue centroids correspond to clusters found in relatively "dust-free" regions of these galaxies, whereas the green centroids correspond to clusters found in relatively dustier regions of the galaxy.

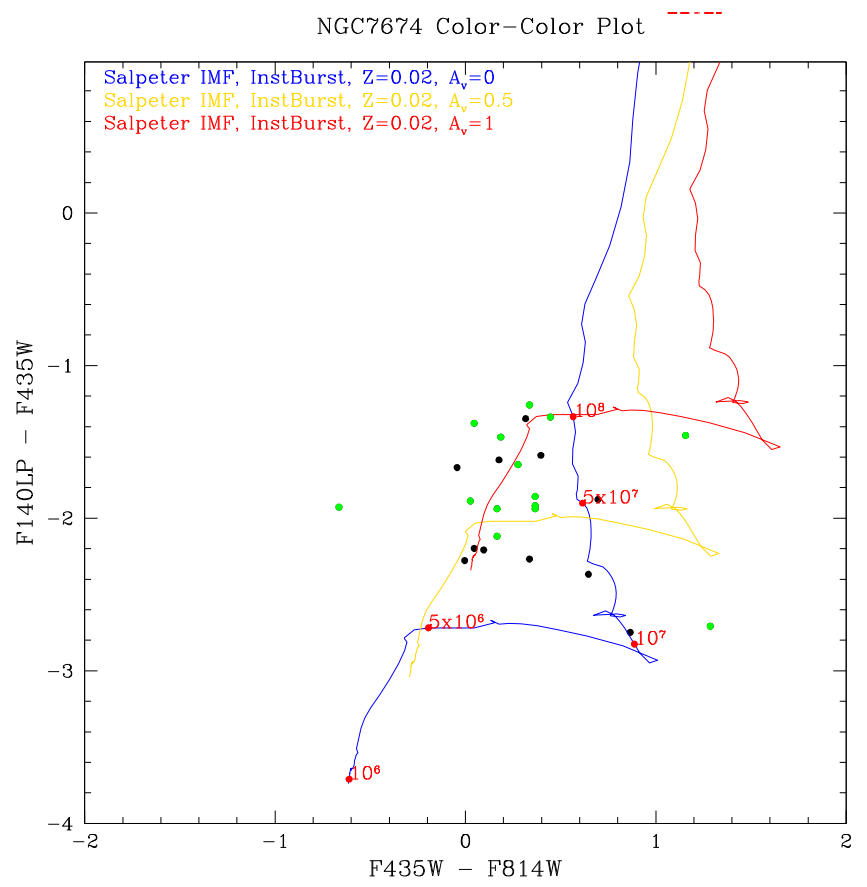

Figure 58. Color-color plot of all star clusters identified in NGC 7674 in F814W, F435W, and F140LP plotted against SSP models with various amounts of visual extinction. The green points correspond to the clusters found in dustier regions of the galaxy in Figure 57. 


\section{References}

Adamo, A., \& Bastian, N. 2015, arXiv:1511.08212

Alonso-Herrero, A., Engelbracht, C. W., Rieke, M. J., Rieke, G. H., \& Quillen, A. C. 2001, ApJ, 546, 952

Anders, P., Bissantz, N., Boysen, L., de Grijs, R., \& Fritze-v. Alvensleben, U. 2007, MNRAS, 377, 91

Armus, L., Mazzarella, J. M., Evans, A. S., et al. 2009, PASP, 121, 559

Bastian, N. 2008a, in ASP Conf. Ser. 390, Pathways Through an Eclectic Universe, ed. J. H. Knapen, T. J. Mahoney, \& A. Vazdekis (San Francisco, CA: ASP), 47

Bastian, N. 2008b, MNRAS, 390, 759

Bastian, N. 2016, in EAS Pub. Ser. 80, Stellar Clusters: Benchmarks of Stellar Physics and Galactic Evolution, ed. E. Moraux, Y. Lebreton, \& C. Charbonnel (Les Ulis: EDP), 5

Bastian, N., Emsellem, E., Kissler-Patig, M., \& Maraston, C. 2006, A\&A, 445, 471

Bastian, N., Schweizer, F., Goudfrooij, P., Larsen, S. S., \& Kissler-Patig, M. 2013, MNRAS, 431, 1252

Bastian, N., Adamo, A., Gieles, M., et al. 2012, MNRAS, 419, 2606

Bertin, E., \& Arnouts, S. 1996, A\&AS, 117, 393

Bruzual, G., \& Charlot, S. 2003, MNRAS, 344, 1000

Calzetti, D., Armus, L., Bohlin, R. C., et al. 2000, ApJ, 533, 682

Calzetti, D., Kinney, A. L., \& Storchi-Bergmann, T. 1994, ApJ, 429, 582

Chandar, R., Fall, S. M., \& Whitmore, B. C. 2010, ApJ, 711, 126

Chandar, R., Fall, S. M., \& Whitmore, B. C. 2015, ApJ, 810, 1

Chien, L. 2010, in ASP Conf. Ser. 423, Galaxy Wars: Stellar Populations and Star Formation in Interacting Galaxies, ed. B. Smith et al. (San Francisco, CA: ASP), 197

Chien, L.-H., Barnes, J. E., Kewley, L. J., \& Chambers, K. C. 2007, ApJL, 660, L105

Dametto, N. Z., Riffel, R., Pastoriza, M. G., et al. 2014, MNRAS, 443, 1754

de Grijs, R., Anders, P., Bastian, N., et al. 2003, MNRAS, 343, 1285

Evans, A. S., Vavilkin, T., Pizagno, J., et al. 2008, ApJL, 675, L69

Fall, S. M., \& Chandar, R. 2012, ApJ, 752, 96

Fall, S. M., Chandar, R., \& Whitmore, B. C. 2005, ApJL, 631, L133

Fall, S. M., Chandar, R., \& Whitmore, B. C. 2009, ApJ, 704, 453

Fitzpatrick, E. L. 1999, PASP, 111, 63

Fouesneau, M., Lançon, A., Chandar, R., \& Whitmore, B. C. 2012, ApJ, 750,60

García-Marín, M., Colina, L., Arribas, S., Alonso-Herrero, A., \& Mediavilla, E. 2006, ApJ, 650, 850

Gieles, M., Bastian, N., Lamers, H. J. G. L. M., \& Mout, J. N. 2005, A\&A, 441, 949

Girardi, L., Bertelli, G., Bressan, A., et al. 2002, A\&A, 391, 195

Gnedin, O. Y., \& Ostriker, J. P. 1997, ApJ, 474, 223

Goddard, Q. E., Bastian, N., \& Kennicutt, R. C. 2010, MNRAS, 405, 857

González Delgado, R. M., Cerviño, M., Martins, L. P., Leitherer, C., \& Hauschildt, P. H. 2005, MNRAS, 357, 945

González-Martín, O., Masegosa, J., Márquez, I., \& Guainazzi, M. 2009, ApJ, 704,1570

Haan, S., Armus, L., Surace, J. A., et al. 2013, MNRAS, 434, 1264

Harris, J., \& Zaritsky, D. 2009, AJ, 138, 1243

Homeier, N., Gallagher, J. S., III, \& Pasquali, A. 2002, A\&A, 391, 857

Hopkins, P. F., Cox, T. J., Hernquist, L., et al. 2013, MNRAS, 430, 1901

Howell, J. H., Armus, L., Mazzarella, J. M., et al. 2010, ApJ, 715, 572

Humphreys, R. M., \& Davidson, K. 1979, ApJ, 232, 409

Hunter, D. A., Elmegreen, B. G., Dupuy, T. J., \& Mortonson, M. 2003, AJ, 126,1836

Inami, H., Armus, L., Surace, J. A., et al. 2010, AJ, 140, 63

Johansson, L., \& Bergvall, N. 1988, A\&A, 192, 81

Johnson, L. C., Seth, A. C., Dalcanton, J. J., et al. 2016, ApJ, 827, 33

Johnson, L. C., Seth, A. C., Dalcanton, J. J., et al. 2017, ApJ, 839, 78

Joy, M., \& Harvey, P. M. 1987, ApJ, 315, 480

Karl, S. J., Fall, S. M., \& Naab, T. 2011, ApJ, 734, 11

Kennicutt, R. C., Jr., Hao, C.-N., Calzetti, D., et al. 2009, ApJ, 703, 1672

Kewley, L. J., Rupke, D., Zahid, H. J., Geller, M. J., \& Barton, E. J. 2010, ApJL, 721, L48

Kim, D.-C., Evans, A. S., Vavilkin, T., et al. 2013, ApJ, 768, 102

King, I. R. 1966, AJ, 71, 276
Kruijssen, J. M. D. 2012, MNRAS, 426, 3008

Kruijssen, J. M. D. 2014, CQGra, 31, 244006

Kruijssen, J. M. D., \& Bastian, N. 2016, MNRAS, 457, L24

Kruijssen, J. M. D., Pelupessy, F. I., Lamers, H. J. G. L. M., Portegies Zwart, S. F., \& Icke, V. 2011, MNRAS, 414, 1339

Kruijssen, J. M. D., Pelupessy, F. I., Lamers, H. J. G. L. M., et al. 2012, MNRAS, 421, 1927

Larsen, S. S. 1999, A\&AS, 139, 393

Larsen, S. S. 2009, A\&A, 494, 539

Larsen, S. S. 2010, RSPTA, 368, 867

Larsen, S. S., \& Richtler, T. 2000, A\&A, 354, 836

Leech, K. J., Penston, M. V., Terlevich, R., et al. 1989, MNRAS, 240, 349

Leitherer, C., Schaerer, D., Goldader, J. D., et al. 1999, ApJS, 123, 3

Li, H., Gnedin, O. Y., Gnedin, N. Y., et al. 2017, ApJ, 834, 69

Lutz, D. 1992, A\&A, 259, 462

Magnelli, B., Elbaz, D., Chary, R. R., et al. 2011, A\&A, 528, A35

Maíz Apellániz, J., \& Úbeda, L. 2005, ApJ, 629, 873

Maji, M., Zhu, Q., Li, Y., et al. 2016, ApJ, submitted (arXiv:1606.07091)

Maoz, D., Barth, A. J., Ho, L. C., Sternberg, A., \& Filippenko, A. V. 2001, AJ, 121,3048

Maraston, C., Bastian, N., Saglia, R. P., et al. 2004, A\&A, 416, 467

Martin, J. M., Bottinelli, L., Gouguenheim, L., \& Dennefeld, M. 1991, A\&A, 245,393

Meurer, G. R., Heckman, T. M., Leitherer, C., et al. 1995, AJ, 110, 2665

Miller, B. W., Whitmore, B. C., Schweizer, F., \& Fall, S. M. 1997, AJ, 114,2381

Miralles-Caballero, D., Colina, L., \& Arribas, S. 2012, A\&A, 538, A61

Momjian, E., Romney, J. D., Carilli, C. L., \& Troland, T. H. 2003, ApJ, 597, 809

Mulia, A. J., Chandar, R., \& Whitmore, B. C. 2015, ApJ, 805, 99

Pasquali, A., de Grijs, R., \& Gallagher, J. S. 2003, MNRAS, 345, 161

Privon, G. C., Barnes, J. E., Evans, A. S., et al. 2013, ApJ, 771, 120

Reines, A. E., Johnson, K. E., \& Goss, W. M. 2008, AJ, 135, 2222

Relaño, M., Lisenfeld, U., Pérez-González, P. G., Vílchez, J. M., \& Battaner, E. 2007, ApJL, 667, L141

Rich, J. A., Torrey, P., Kewley, L. J., Dopita, M. A., \& Rupke, D. S. N. 2012, ApJ, 753, 5

Rupke, D. S. N., Veilleux, S., \& Baker, A. J. 2008, ApJ, 674, 172

Salpeter, E. E. 1955, ApJ, 121, 161

Sanders, D. B., Mazzarella, J. M., Kim, D.-C., Surace, J. A., \& Soifer, B. T 2003, AJ, 126, 1607

Sanders, D. B., \& Mirabel, I. F. 1996, ARA\&A, 34, 749

Schlafly, E. F., \& Finkbeiner, D. P. 2011, ApJ, 737, 103

Schweizer, F., Miller, B. W., Whitmore, B. C., \& Fall, S. M. 1996, AJ, 112,1839

Schweizer, F., \& Seitzer, P. 1998, AJ, 116, 2206

Silva-Villa, E., Adamo, A., Bastian, N., Fouesneau, M., \& Zackrisson, E. 2014, MNRAS, 440, L116

Sirianni, M., Jee, M. J., Benítez, N., et al. 2005, PASP, 117, 1049

Smith, B. J., Soria, R., Struck, C., et al. 2014, AJ, 147, 60

Stierwalt, S., Armus, L., Surace, J. A., et al. 2013, ApJS, 206, 1

Trancho, G., Bastian, N., Schweizer, F., \& Miller, B. W. 2007, ApJ, 658, 993

Tutukov, A. V. 1978, A\&A, 70, 57

U, V., Medling, A., Sanders, D., et al. 2013, ApJ, 775, 115

van den Broek, A. C., van Driel, W., de Jong, T., et al. 1991, A\&A, 91, 61

van Driel, W., Gao, Y., \& Monnier-Ragaigne, D. 2001, A\&A, 368, 64

Vavilkin, T. 2011, PhD thesis, Stony Brook Univ.

Whitmore, B., Chandar, R., Bowers, A., et al. 2014, AJ, 147, 78

Whitmore, B. C., Chandar, R., \& Fall, S. M. 2007, AJ, 133, 1067

Whitmore, B. C., Chandar, R., Schweizer, F., et al. 2010, AJ, 140, 75

Whitmore, B. C., Miller, B. W., Schweizer, F., \& Fall, S. M. 1997, AJ, 114,1797

Whitmore, B. C., \& Schweizer, F. 1995, AJ, 109, 960

Whitmore, B. C., \& Zhang, Q. 2002, AJ, 124, 1418

Whitmore, B. C., Zhang, Q., Leitherer, C., et al. 1999, AJ, 118, 1551

Wilson, C. D., Harris, W. E., Longden, R., \& Scoville, N. Z. 2006, ApJ, 641, 763

Zepf, S. E., Ashman, K. M., English, J., Freeman, K. C., \& Sharples, R. M. 1999, AJ, 118, 752

Zhang, Q., \& Fall, S. M. 1999, ApJL, 527, L81 